NATIONAL LABORATORY

\title{
Analysis of HFIR Dosimetry Experiments Performed in Cycles 400 and 401
}

September 2008

Prepared by

I. Remec

C. A. Baldwin

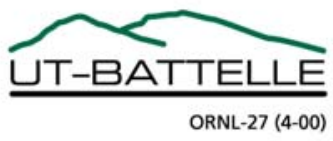




\section{DOCUMENT AVAILABILITY}

Reports produced after January 1, 1996, are generally available free via the U.S. Department of Energy (DOE) Information Bridge.

Web site http://www.osti.gov/bridge

Reports produced before January 1, 1996, may be purchased by members of the public from the following source.

National Technical Information Service

5285 Port Royal Road

Springfield, VA 22161

Telephone 703-605-6000 (1-800-553-6847)

TDD 703-487-4639

Fax 703-605-6900

E-mail info@ntis.gov

Web site http://www.ntis.gov/support/ordernowabout.htm

Reports are available to DOE employees, DOE contractors, Energy Technology Data Exchange (ETDE) representatives, and International Nuclear Information System (INIS) representatives from the following source.

Office of Scientific and Technical Information

P.O. Box 62

Oak Ridge, TN 37831

Telephone 865-576-8401

Fax 865-576-5728

E-mail reports@osti.gov

Web site http://www.osti.gov/contact.html

This report was prepared as an account of work sponsored by an agency of the United States Government. Neither the United States Government nor any agency thereof, nor any of their employees, makes any warranty, express or implied, or assumes any legal liability or responsibility for the accuracy, completeness, or usefulness of any information, apparatus, product, or process disclosed, or represents that its use would not infringe privately owned rights. Reference herein to any specific commercial product, process, or service by trade name, trademark, manufacturer, or otherwise, does not necessarily constitute or imply its endorsement, recommendation, or favoring by the United States Government or any agency thereof. The views and opinions of authors expressed herein do not necessarily state or reflect those of the United States Government or any agency thereof. 
Nuclear Science and Technology Division

\section{ANAL YSIS OF HFIR DOSIMETRY EXPERIMENTS PERFORMED IN CYCLES 400 AND 401}

I. Remec and C. A. Baldwin

Date Published: September 2008

Prepared by

OAK RIDGE NATIONAL LABORATORY

P.O. Box 2008

Oak Ridge, Tennessee 37831-6285

managed by

UT-Battelle, LLC

for the

U.S. DEPARTMENT OF ENERGY

under contract DE-AC05-00OR22725 



\section{CONTENTS}

Page

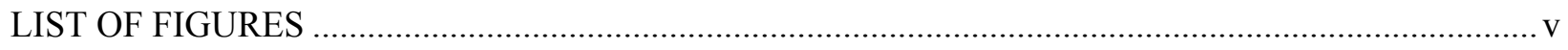

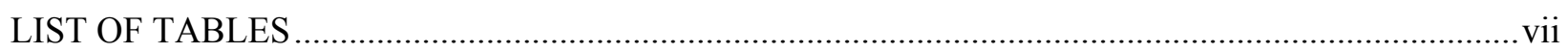

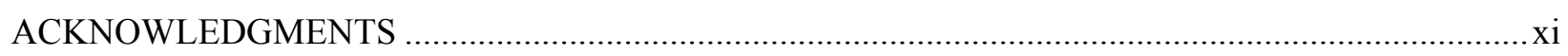

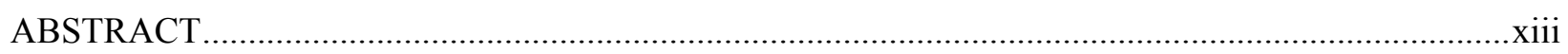

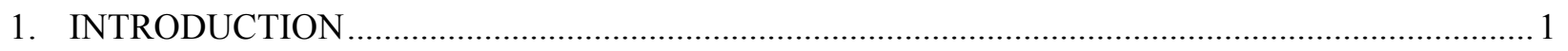

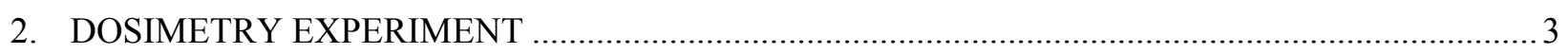

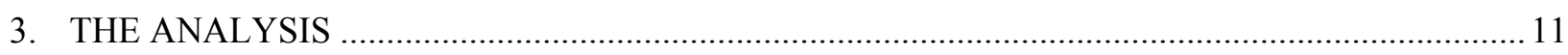

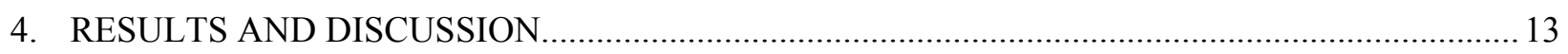

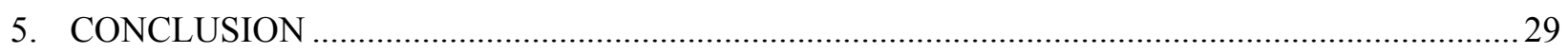

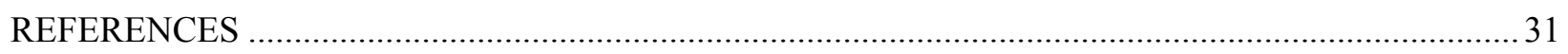

APPENDIX A: MEASURED SPECIFIC ACTIVITIES AND HELIUM

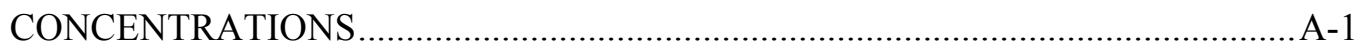

APPENDIX B: MULTIGROUP NEUTRON AND GAMMA-RAY FLUXES

OBTAINED FROM TRANSPORT CALCULATIONS .......................................... B-1

APPENDIX C: COMPARISON OF ADJUSTED IRRADIATION PARAMETERS

FROM DIFFERENT ADJUSTMENT RUNS AND ADDITIONAL

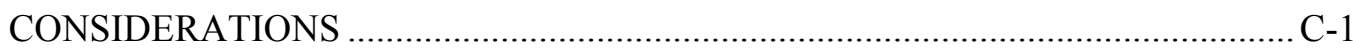

APPENDIX D: ADJUSTED IRRADIATION PARAMETERS AS GIVEN IN THE

PRELIMINARY ANALYSIS REPORT, DATED NOVEMBER 18, 2005 ................ D-1

APPENDIX E: EFFECT OF NEUTRON AND GAMMA FLUX CHANGES.................................. E-1

APPENDIX F: COMPUTER CODES AND DATA ARCHIVE....................................................... F-1 



\section{LIST OF FIGURES}

Figure

1 Cross section of HFIR core and PV at core horizontal midplane .........................................2

2 Capsules for HFIR dosimeter experiments.................................................................... 4

3 Schematic view of HFIR surveillance keys with numbered positions of

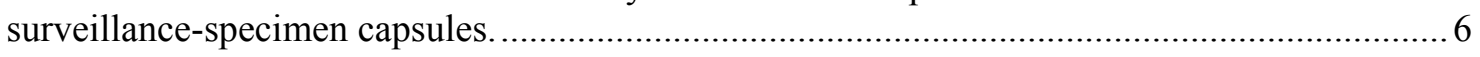

4 Details of the CVN surveillance capsule locations and tubular dosimeters

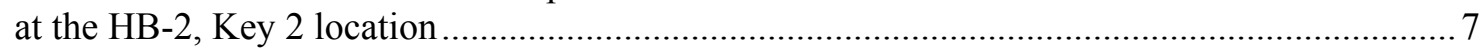

5 Schematic showing the location and orientation of CVN specimens for HFIR upgraded beam tubes with respect to the nozzle weld and corner. ............................... 8

6 Schematic of HB-2 surveillance Keys $2 \mathrm{C}$ and $2 \mathrm{~W}$ and the locations of the

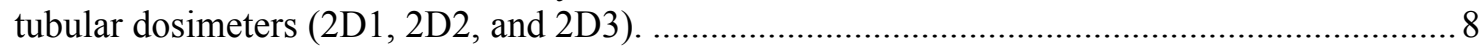

7 Vertical cross section of Key 2 showing the location of CVN specimens and

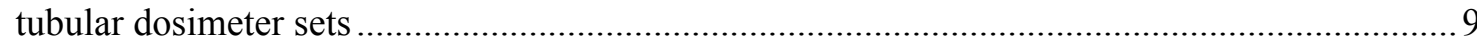

8 Locations and labeling of the dosimeter vials in the tubular dosimeter capsules at the HB-2 Key 2D location..

C.1 Comparison of the adjusted irradiation parameters at the dosimeter locations inside the tubular dosimeter sets located at Key 2. 



\section{LIST OF TABLES}

Table

Page

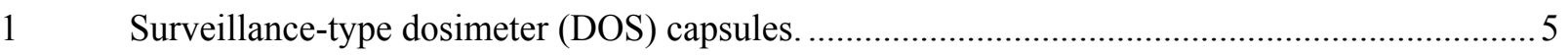

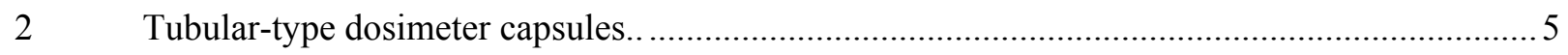

3 Uncertainties of the measured reaction rates (as used in the adjustment calculations) .............. 12

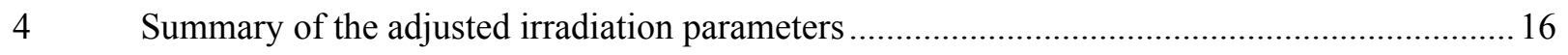

$5 \quad$ Calculated and adjusted irradiation parameters at K2W, P8 (capsule DOS-1) ........................ 17

6 Calculated and adjusted irradiation parameters at K2W, P32 (capsule DOS-2) ...................... 18

$7 \quad$ Calculated and adjusted irradiation parameters at K3, P3 (capsule DOS-3) ............................ 19

$8 \quad$ Calculated and adjusted irradiation parameters at K3, P8 (capsule DOS-4) ........................... 20

9 Calculated and adjusted irradiation parameters at K3, P10 (capsule DOS-5) ........................2 21

10 Calculated and adjusted irradiation parameters at K7, P1 (capsule DOS-6) ...........................22

11 Calculated and adjusted irradiation parameters at K2C, P1 (capsule DOS-8) .......................23

12 Calculated and adjusted irradiation parameters at K2C, P4 (capsule DOS-9) ........................22

13 Calculated and adjusted irradiation parameters at 2D1W (capsules HRB2-1G and HRB2-1A, position 4; dosimeter sets GD-15 and AL-28; HRB2-1W) ............................. 24

14 Calculated and adjusted irradiation parameters at 2D2W (capsules HRB2-2G and HRB2-2A, position 4; dosimeter sets GD-17 and AL-30; HRB2-2W) ............................. 24

15 Calculated and adjusted irradiation parameters at 2D3W (capsules HRB2-3G

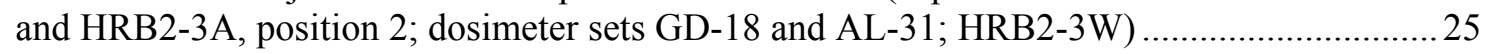

16 Calculated and adjusted irradiation parameters at 2D2C (capsules HRB2-2G

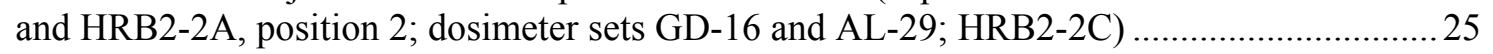

17 Calculated and adjusted irradiation parameters at 2D1C (capsules HRB2-1G and HRB2-1A, position 2; dosimeter sets GD-14 and AL-27; HRB2-1C) ............................. 26

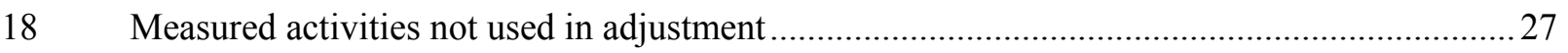

A.1 Measured specific activities of the dosimeters irradiated during HFIR fuel cycle 400............ A-3

A.2 Measured specific activities of the dosimeters irradiated during HFIR fuel cycle 401 ............ A-9

A.3 Measured helium concentrations in Be HAFMs ................................................................. A-10

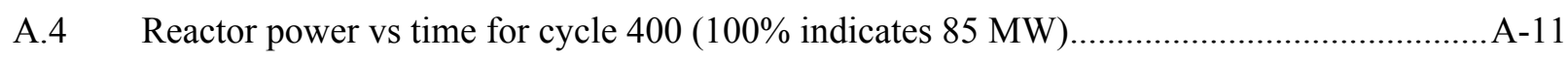

A.5 Reactor power vs time for cycle 401 (100\% indicates $85 \mathrm{MW})$...........................................

B.1 Energy boundaries for 61 neutron groups used in transport calculations $(\mathrm{eV})$..................... B-3

B.2 Energy boundaries for 23 gamma-ray groups used in transport calculations (eV) ................ B-3

B.3 Calculated neutron and gamma fluxes normalized to the reactor power of 85 MW for locations K2W, P8 (DOS-1) and K2W, P32 (DOS-2) ....................................... B-4

B.4 Calculated neutron and gamma fluxes normalized to the reactor power of 85 MW for location K3, P3 (DOS-3). 


\section{LIST OF TABLES (continued)}

Table

Page

B.5 Calculated neutron and gamma fluxes normalized to the reactor power of $85 \mathrm{MW}$ for location K3, P8 (DOS-4).

B.6 Calculated neutron and gamma fluxes normalized to the reactor power of 85 MW for location K3, P10 (DOS-5).

B.7 Calculated neutron and gamma fluxes normalized to the reactor power of $85 \mathrm{MW}$ for location K7, P1 (DOS-6).

B.8 Calculated neutron and gamma fluxes normalized to the reactor power of $85 \mathrm{MW}$ for locations K2C, P1 (DOS-8), and K2C, P4 ( DOS-9).

B.9 Calculated neutron and gamma fluxes normalized to the reactor power of $85 \mathrm{MW}$ for locations 2D1W, 2D2W, and 2D3W....

C.1 Comparison of the adjusted irradiation parameters for capsule DOS-4 ............................... C-6

C.2 Comparison of adjusted irradiation parameters for capsule DOS-8, at K2C, P1, for several adjustment runs.

C.3 Comparison of adjusted irradiation parameters for capsule DOS-9, at K2C, P4, for several adjustment runs.

C.4 Comparison of adjusted irradiation parameters at location 2D2C for different adjustment runs.

C.5 Comparison of adjusted irradiation parameters at location 2D1C for different adjustment runs.

C.6 Comparison of measured specific activities at the two beam line 2 nozzle corner locations, 2D1C and 2D2C.

C.7 Comparison of calculated and "measured" reaction rates for the Key 2, positions 2D1C and 2D2C.

C.8 Calculated (obtained from transport calculations) and modified ("new") flux values for the top five energy groups, for the location 2D1C

C.9 Comparison of two adjustment runs for location 2D1C ................................................ C-12

D.1 Summary of the adjusted irradiation parameters from the preliminary analysis ....................D-4

D.2 Calculated (for Position 2) and adjusted irradiation parameters for capsule DOS-1, at $\mathrm{K} 2 \mathrm{~W}, \mathrm{P} 8$ from the preliminary analysis and comparison with the final analysis.

D.3 Calculated (for Position 2 only) and adjusted irradiation parameters for capsule DOS-2, at K2W, P3 from the preliminary analysis and comparison with the final analysis

D.4 Calculated (for Position 2 only) and adjusted irradiation parameters for capsule DOS-3, at K3, P3 from the preliminary analysis and comparison with the final analysis

D.5 Calculated (for Position 2 only) and adjusted irradiation parameters for capsule DOS-4, at K3, P8 from the preliminary analysis and comparison with the final analysis 


\section{LIST OF TABLES (continued)}

\section{Table}

Page

D.6 Calculated (for Position 2 only) and adjusted irradiation parameters for capsule DOS-5, at K3, P10 from the preliminary analysis and comparison with the final analysis D-9

E.1 Calculated neutron and gamma fluxes at the location 2D3W …........................................ E-4

E.2 Calculated neutron and gamma fluxes at the locations 2D1C and 2D2C........................... E-5

E.3 Calculated and adjusted irradiation parameters at 2D3W ............................................... E-6

E.4 Calculated and adjusted irradiation parameters at 2D1C ................................................ E-7

E.5 Calculated and adjusted irradiation parameters at 2D2C ............................................... E-8

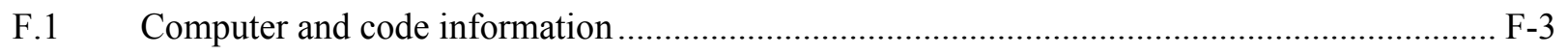

F.2 Content of the archive directory "Dosimetry" ............................................................. F-4 



\section{ACKNOWLEDGMENTS}

The authors wish to acknowledge the contributions of E. D. Blakeman, who performed neutron and gamma-ray transport calculations, D. C. Glasgow, who carried out measurements of activation and fission dosimeters, and B. M. Oliver (Pacific Northwest National Laboratory), who measured the helium content of beryllium dosimeters. Special thanks also to R. D. Cheverton, E. D. Blakeman, and H. A.

Kmieciak, Jr., who provided numerous comments and suggested improvements to this report and to C. O. Slater, who performed an independent review of this report. 



\begin{abstract}
The High Flux Isotope Reactor (HFIR) has been in operation at Oak Ridge National Laboratory since 1966. To upgrade and enhance capabilities for neutron science research at the reactor, a larger HB-2 beam tube was installed in April of 2002. To assess, experimentally, the impact of this larger beam tube on radiation damage rates [i.e., displacement-per-atom (dpa) rates] used in vessel life extension studies, dosimetry experiments were performed from April to August 2004 during fuel cycles 400 and 401 . This report documents the analysis of the dosimetry experiments and the determination of best-estimate dpa rates. These dpa rates are obtained by performing a least-squares adjustment of calculated neutron and gamma-ray fluxes and the measured responses of radiometric monitors and beryllium helium accumulation fluence monitors. The best-estimate dpa rates provided here will be used to update HFIR pressure vessel life extension studies, which determine the pressure/temperature limits for reactor operation and the HFIR pressure vessel's remaining life. All irradiation parameters given in this report correspond to a reactor power of $85 \mathrm{MW}$.
\end{abstract}





\section{INTRODUCTION}

The High Flux Isotope Reactor (HFIR) was designed and built at Oak Ridge National Laboratory (ORNL) in the late 1950s and early 1960s. A comprehensive pressure vessel (PV) surveillance program was developed to check and monitor the radiation-induced damage to the pressure vessel material and the resulting impact on the safety limits for operation of the vessel, as well as on the vessel life. ${ }^{1}$ The surveillance program uses Charpy V-notch $(\mathrm{CVN})$ specimens of vessel material and radiometric monitors (dosimeters) located at the PV inner wall and at the beam-line nozzles as shown in Fig. 1. Seven surveillance locations, referred to as "keys," are used. At each key, capsules containing Charpy specimens are irradiated.

Surveillance capsules were removed from HFIR in 1969, 1974, 1983, and 1986. The 1983 and 1986 testing of the CVN specimens revealed greater-than-expected change in the Nil-Ductility Transition Temperature (NDTT). As a result, the HFIR operating pressure was lowered, requiring a reduction in reactor power from $100 \mathrm{MW}$ to $85 \mathrm{MW}^{2}$ In addition, periodic hydrostatic proof testing was introduced, additional capsules of vessel material were installed, and the capsule removal schedule was modified. In 1993, research into the cause of the higher-than-expected radiation damage rates identified gamma radiation as the reason for additional damage. ${ }^{3}$

In late 1990, an initiative to increase the diameter of horizontal beam line 2 (HB-2) and HB-4 was launched, with the provision that the increase would not reduce PV lifetime excessively. A new PV lifetime study ${ }^{4}$, using techniques proposed in $1993,{ }^{5}$ indicated that by updating criteria and methods of analysis 50 EFPY $(100 \mathrm{MW})$ could be achieved; however, another modification of the PV surveillance program was necessary, and installation of dosimeters sensitive to both neutron and gamma rays was required to determine the radiation damage rates. ${ }^{6}$

The larger HB-2 beam tube was installed during the end-of-cycle 384 outage (April of 2002). The beam tube remained out-of-service until December 2003, at which time it was placed into operation (cycle 398). The dosimetry experiments were performed in 2004 during fuel cycles 400 and 401, which occurred in May and July, respectively.

This report presents the dosimetry analysis methodology and provides best-estimate irradiation parameters obtained from the dosimetry experiments performed during cycles 400 and 401. 


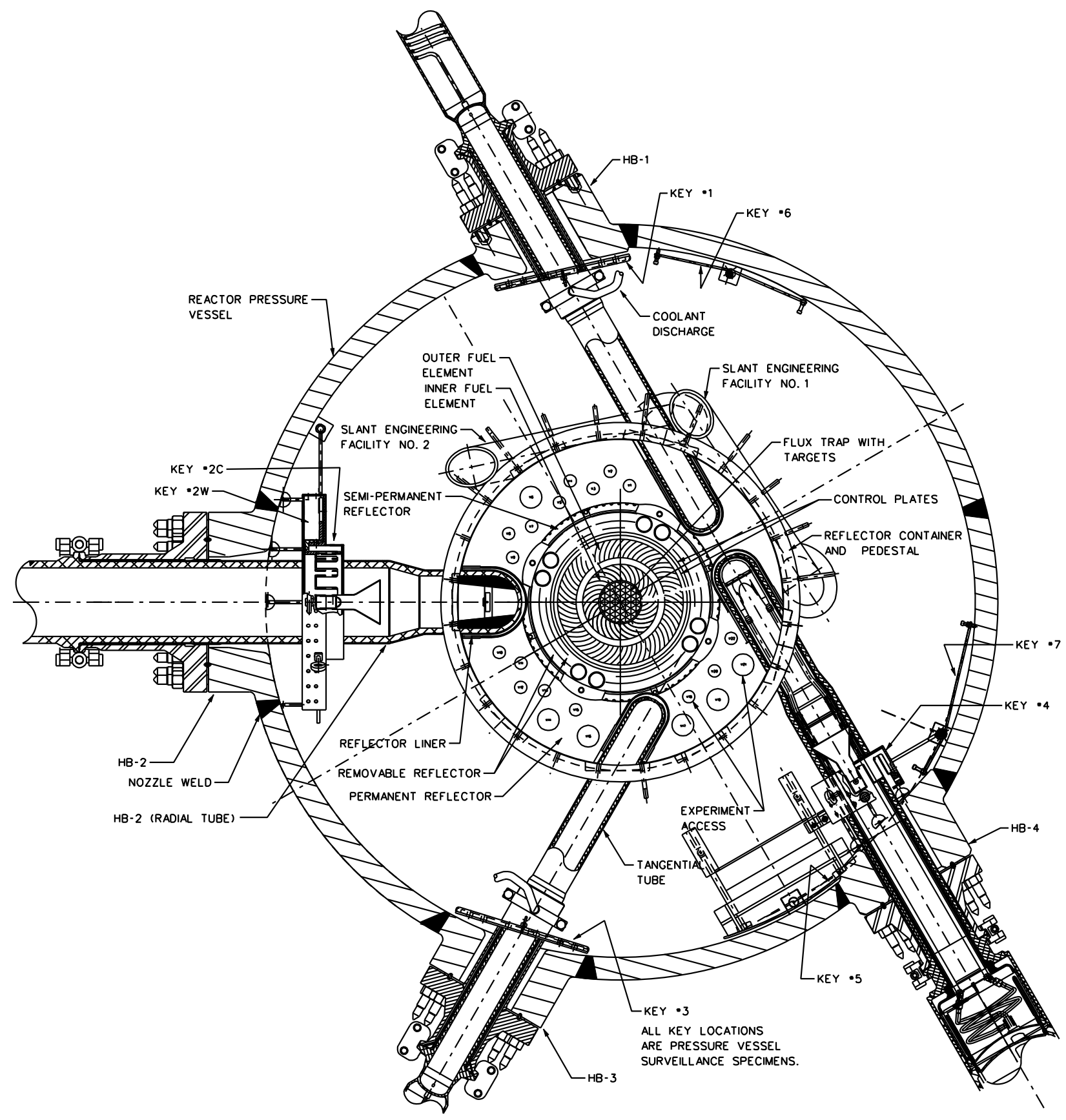

Fig. 1. Cross section of HFIR core and PV at core horizontal midplane. The locations of surveillance specimens-"keys"-are numbered 1-7. (Note: HB-4 was not installed at the time of the HB-2 dosimetry experiment.) 


\section{DOSIMETRY EXPERIMENT}

The dosimetry experiment performed during cycles 400 and 401 used a total of 137 activation dosimeters and 13 beryllium helium accumulation fluence monitors (Be HAFMs). The dosimeters were placed in either aluminum or gadolinium vials to allow or eliminate exposure to thermal neutrons, respectively. Each gadolinium vial included ${ }^{237} \mathrm{NpO}$, Be HAFM, Ni and Co wire, and some vials included a diluted cobalt wire in the form of $0.1 \mathrm{wt} \% \mathrm{Co} / \mathrm{Al}$ alloy. Each aluminum vial contained $\mathrm{Cu}, \mathrm{Ni}, \mathrm{Fe}$, and $\mathrm{Co}$ wire, and some vials contained a diluted $\mathrm{Co}$ wire in the form of $0.1 \mathrm{wt} \% \mathrm{Co} / \mathrm{Al}$ alloy.

Two types of capsules were used to hold the dosimeter vials: surveillance-type dosimeter (DOS) capsules and tubular capsules, as shown in Fig. 2. A DOS capsule is identical to the standard CVN surveillance capsule except that it is filled with a pair of stainless steel inserts that have three slots into which the dosimeter vials are placed. For each DOS capsule, a gadolinium vial was placed in the central position, and aluminum vials were placed in the two peripheral positions. Two types of DOS capsules were used. Type "A" DOS capsules had horizontal slots for the vials, while type "B" capsules had vertical vial slots. Type "A" capsules were used in keys where the capsules are placed perpendicular to the beam tube, while type "B" capsules were positioned parallel to the beam tubes. This was done to minimize the flux gradient across a given dosimeter vial. Tubular dosimeter capsules were long tubes with two positions for dosimeter vials. In certain tubular capsules, only the bottom location was used for dosimeters. Complete information about the dosimetry capsules and the dosimeters can be found in Ref. 7. Summary information about the surveillance capsules and the locations at which they were irradiated in HFIR is given in Tables 1 and 2.

The positions of the dosimetry capsules inside the HFIR PV during irradiation are shown in Figs. 3-8. Figure 3 schematically shows the HFIR surveillance keys and displays the locations of the DOS capsules in Key 7 and Key 3. Most of the capsules were irradiated in the carousel around HB-2 to assess the effect of the larger beam tube. Figure 4 shows the positions of Key $2 \mathrm{C}$ and Key $2 \mathrm{~W}$ as viewed from the HFIR core. The locations of the DOS capsules are shown, and the locations of the holders for the tubular capsules are indicated. Figure 5 shows schematically the positions of Charpy specimens in the two HB-2 keys relative to the location of the nozzle corner and weld. Figure 6 presents the horizontal cross section of the HB-2 nozzle area, with locations of the Keys 2C and 2W and the holders for the tubular capsules. Figure 7 shows the view from the side of the HB-2 nozzle area and the locations of Keys 2C, 2W, and the holders for the tubular capsules. Finally, Fig. 8 shows schematically the locations of dosimeter capsules in the tubular capsules with respect to the nozzle corner and weld at HB-2.

After the irradiation, the activation and fission dosimeters were measured with high-resolution gammaray spectrometers at the Neutron Activation Analysis Facility of the Chemical Sciences Division. The complete report is given in Ref. 8. The measured specific activities are listed in Appendix A in Tables A.1 and A.2. The dosimeter activities are grouped by irradiation locations; all activities measured "at the same location" are listed in one group. The helium concentrations in the Be HAFMs were measured at Pacific Northwest National Laboratory; the complete report is given in Ref. 9, and the results are summarized in Table A.3 in Appendix A.

The HFIR reactor power vs time histories for fuel cycles 400 and 401 are given in Tables A.4 and A.5 in Appendix A. The power vs time history is needed to convert specific activities to cycle-time-averaged reaction rates. 

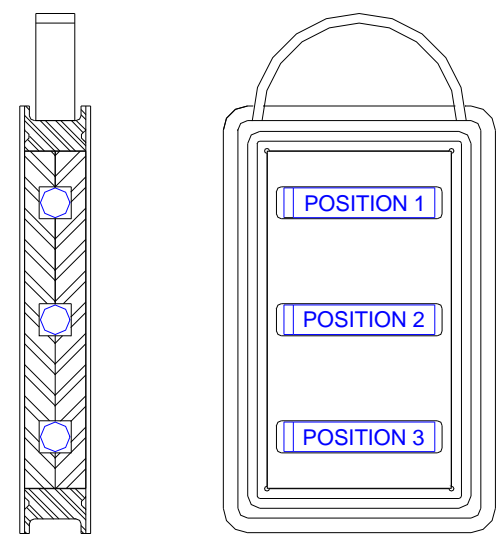

SURVEILLANCE CAPSULE WITH TYPE "A" HOLDER
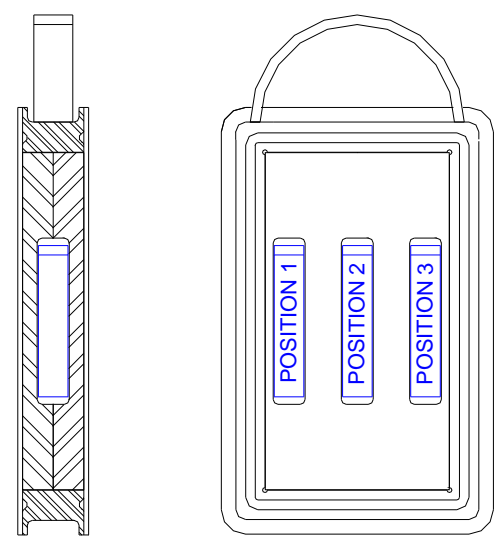

SURVEILLANCE CAPSULE WITH TYPE "B" HOLDER

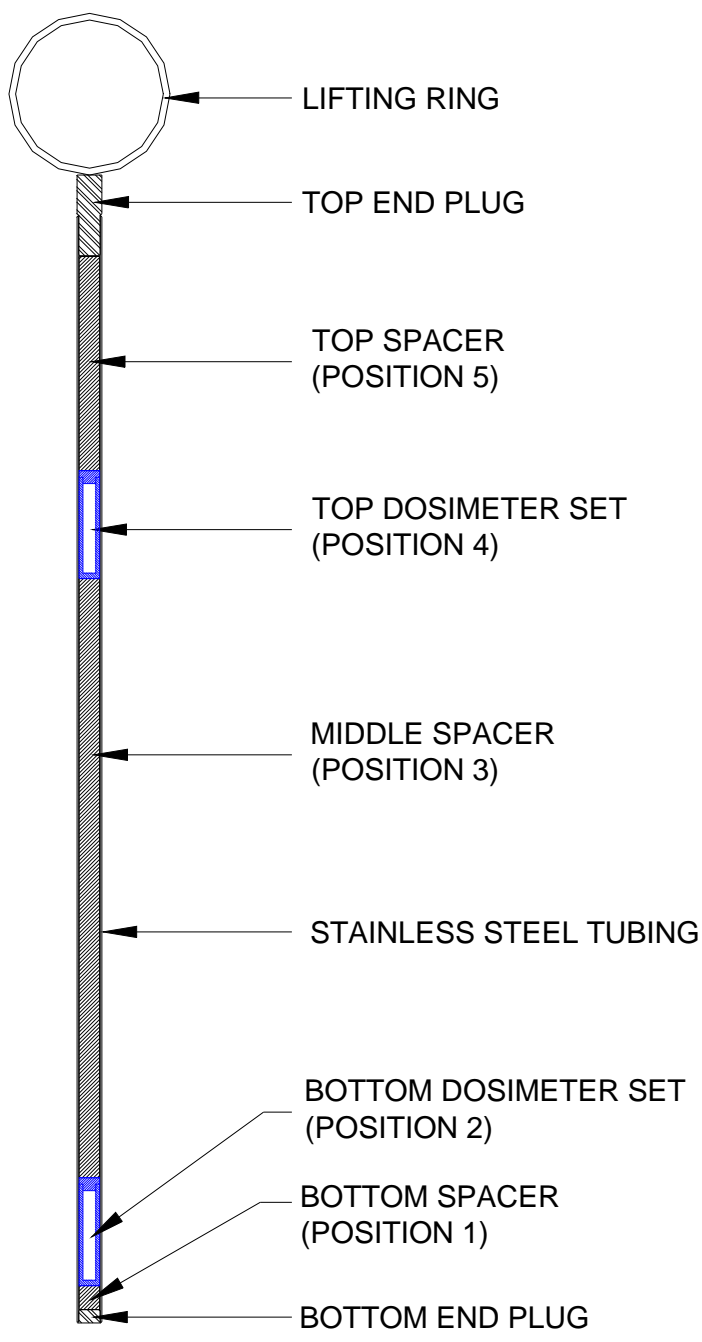

TUBULAR CAPSULE (NOT TO SCALE)

Fig. 2. Capsules for HFIR dosimeter experiments. At left the surveillance-type capsules are shown; at the top is the type " $\mathrm{A}$ " capsule, and at the bottom is the type " $\mathrm{B}$ " capsule. The numbering of the locations for dosimeter vials is shown; orientation of a vial is important in radiation fields with strong gradients. At the right is the tubular capsule. For the tubular capsules, which were installed around the HB-2 beam line, the position 2 (at the bottom) corresponded to the "nozzle corner" location, and the position 4 corresponded to the "nozzle weld" location for capsules inserted into positions 2D1 and 2D2. However, for the tubular capsule installed in position 2D3, the Position 2 location corresponded to the "nozzle weld" 
Table 1. Surveillance-type dosimeter (DOS) capsules.

For each DOS capsule, the following information is provided:

capsule location during irradiation (HFIR key number and position number),

the IDs and locations of the vials containing dosimeters;

the type of capsule; and the HFIR fuel cycle

during which the capsule was irradiated.

\begin{tabular}{cccccccc}
\hline \multirow{2}{*}{$\begin{array}{c}\text { HFIR } \\
\text { key }\end{array}$} & $\begin{array}{c}\text { Key } \\
\text { position }\end{array}$ & Capsule ID & \multicolumn{3}{c}{\begin{tabular}{c} 
Vial ID at \\
\cline { 4 - 5 } Capsule $_{\text {type }^{\boldsymbol{a}}}$
\end{tabular}} & Fuel cycle \\
\hline $2 \mathrm{~W}$ & 8 & DOS-1 & AL-01 & GD-01 & AL-02 & A & 400 \\
$2 \mathrm{~W}$ & 32 & DOS-2 & AL-03 & GD-02 & AL-04 & A & 400 \\
3 & 3 & DOS-3 & AL-05 & GD-03 & AL-06 & A & 400 \\
3 & 8 & DOS-4 & AL-07 & GD-04 & AL-08 & A & 400 \\
3 & 10 & DOS-5 & AL-09 & GD-05 & AL-10 & A & 400 \\
7 & 1 & DOS-6 & AL-11 & GD-06 & AL-12 & A & 400 \\
$2 \mathrm{C}$ & 1 & DOS-8 & AL-15 & GD-08 & AL-16 & B & 400 \\
2 C & 4 & DOS-9 & AL-17 & GD-09 & AL-18 & B & 400 \\
\hline
\end{tabular}

${ }^{a}$ The type (A or B) capsule determines the orientation of the vials in the capsule (see surveillance capsule sketch in Fig. 2).

Table 2. Tubular-type dosimeter capsules.

For each tubular capsule, the following information is provided: capsule location during irradiation;

the IDs and locations of the vials containing dosimeters; and the HFIR fuel cycle during which the capsule was irradiated.

\begin{tabular}{|c|c|c|c|c|c|c|}
\hline \multirow{2}{*}{ Capsule ID } & \multirow{2}{*}{ Key } & \multirow{2}{*}{$\begin{array}{c}\text { Wire- } \\
\text { dosimeter } \\
\text { port }\end{array}$} & \multirow{2}{*}{$\begin{array}{c}\text { Location } \\
\text { ID }^{a}\end{array}$} & \multicolumn{2}{|c|}{ Vial ID at } & \multirow{2}{*}{$\begin{array}{l}\text { Fuel } \\
\text { cycle }\end{array}$} \\
\hline & & & & Position $2^{b}$ & Position $4^{b}$ & \\
\hline HRB2-1G & 2 & 2D1 & $\begin{array}{l}2 \mathrm{D} 1 \mathrm{C} \\
2 \mathrm{D} 1 \mathrm{~W}\end{array}$ & GD-14 & GD-15 & $\begin{array}{l}400 \\
400\end{array}$ \\
\hline HRB2-2G & 2 & 2D2 & $\begin{array}{l}\text { 2D2C } \\
2 \mathrm{D} 2 \mathrm{~W}\end{array}$ & GD-16 & GD-17 & $\begin{array}{l}400 \\
400 \\
\end{array}$ \\
\hline HRB2-3G & 2 & $2 \mathrm{D} 3$ & $2 \mathrm{D} 3 \mathrm{~W}$ & GD-18 & NA & 400 \\
\hline HRB2-1A & 2 & 2D1 & $\begin{array}{l}2 \mathrm{D} 1 \mathrm{C} \\
2 \mathrm{D} 1 \mathrm{~W}\end{array}$ & AL-27 & AL-28 & $\begin{array}{l}401 \\
401\end{array}$ \\
\hline HRB2-2A & 2 & 2D2 & $\begin{array}{l}2 \mathrm{D} 2 \mathrm{C} \\
2 \mathrm{D} 2 \mathrm{~W}\end{array}$ & AL-29 & AL-30 & $\begin{array}{l}401 \\
401 \\
\end{array}$ \\
\hline HRB2-3A & 2 & 2D3 & $2 \mathrm{D} 3 \mathrm{~W}$ & AL-31 & NA & 401 \\
\hline
\end{tabular}

${ }^{a}$ Location IDs are shown in Fig. 8 and are used throughout this report to identify the locations where the irradiation parameters are given.

${ }^{b}$ Positions are shown in the sketch of tubular capsule in Fig. 2. 


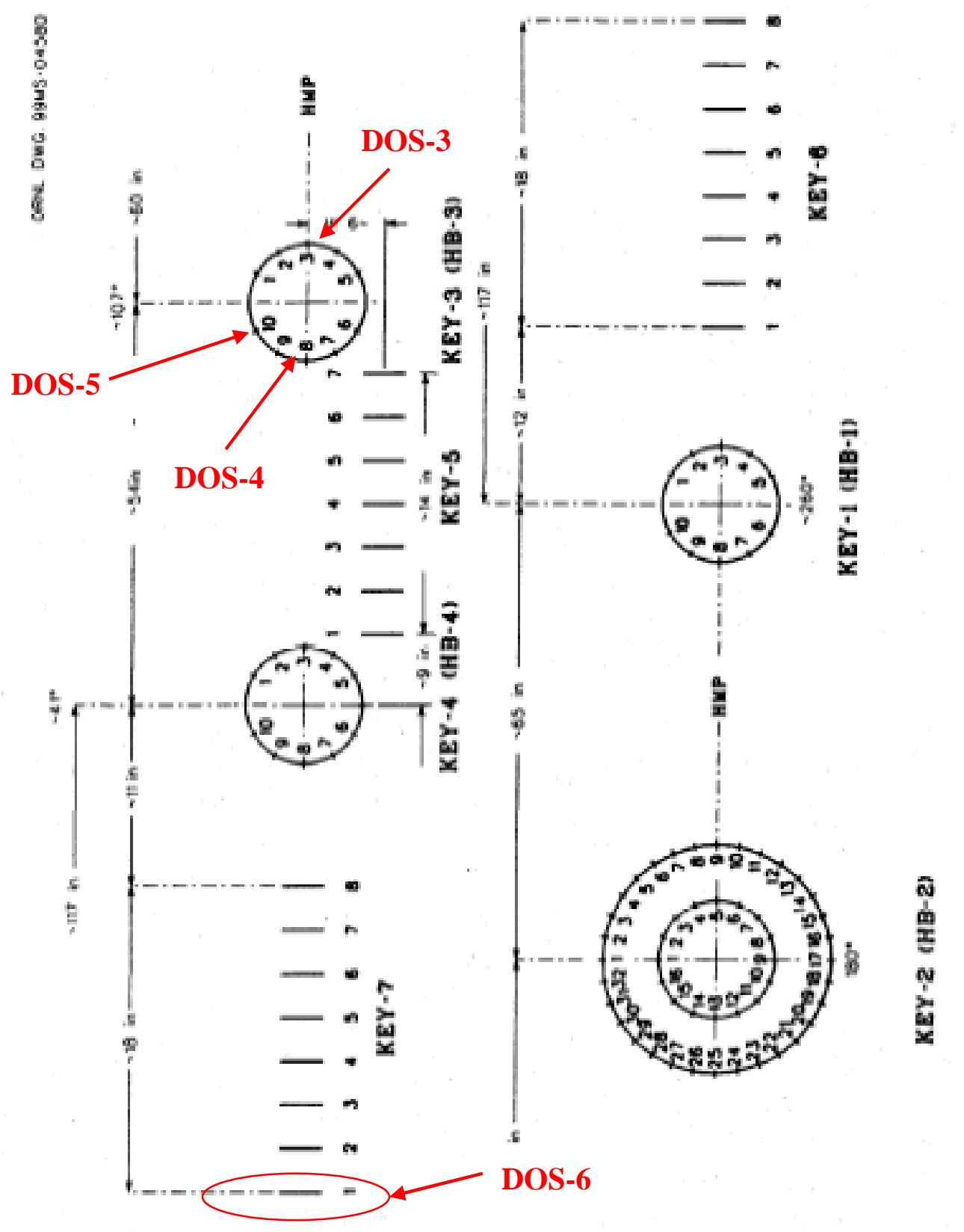

Fig. 3. Schematic view of HFIR surveillance keys with numbered positions of surveillance-specimen capsules. The view is from the core to the PV. Locations of dosimeter capsules used in the dosimetry experiment are shown in red. (The diagram for Key 4 is not current.) 


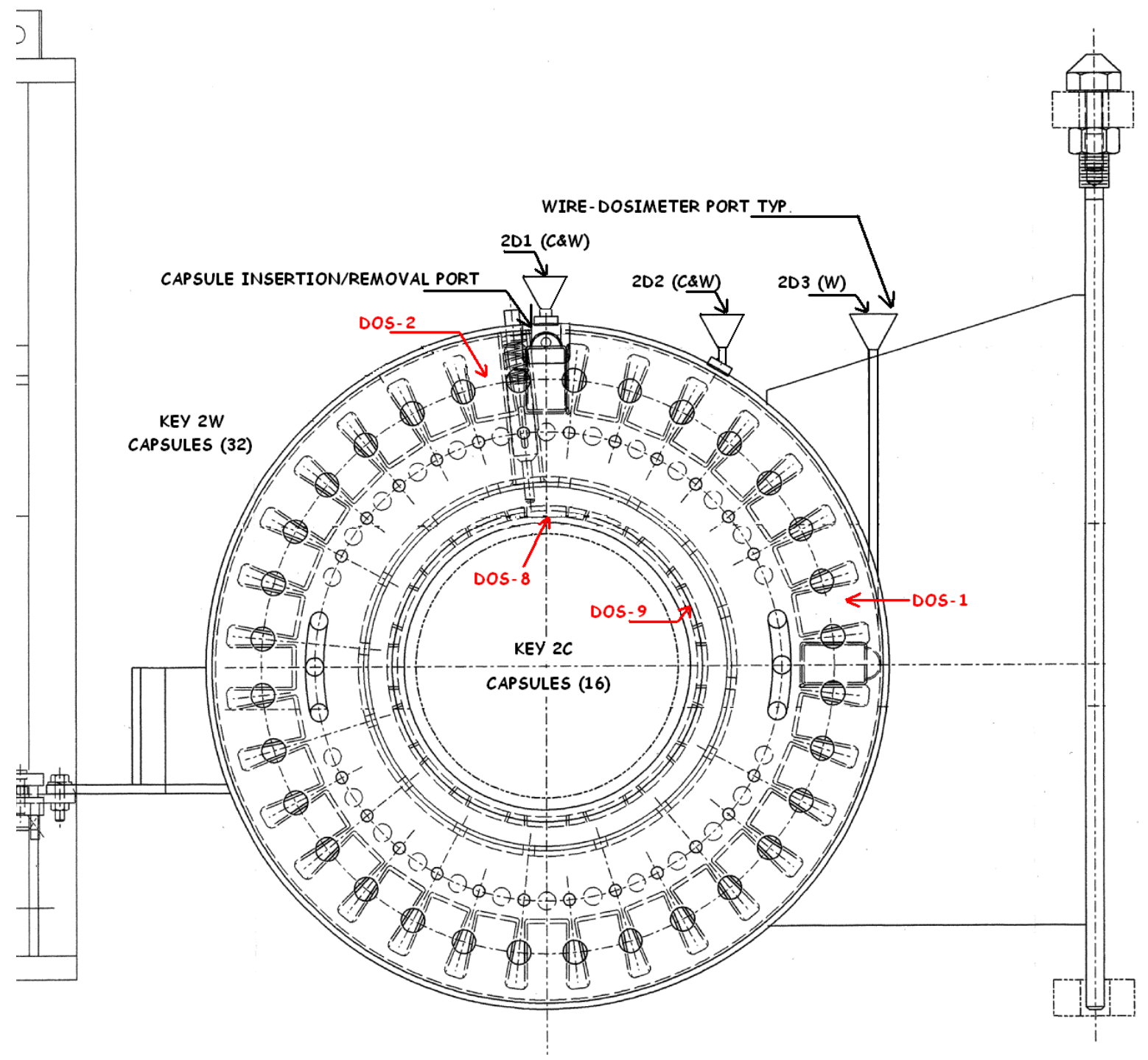

Fig. 4. Details of the CVN surveillance capsule locations and tubular dosimeters at the HB-2, Key 2 location. The view is from the core to the PV. The locations of the dosimeter capsules are shown in red. 


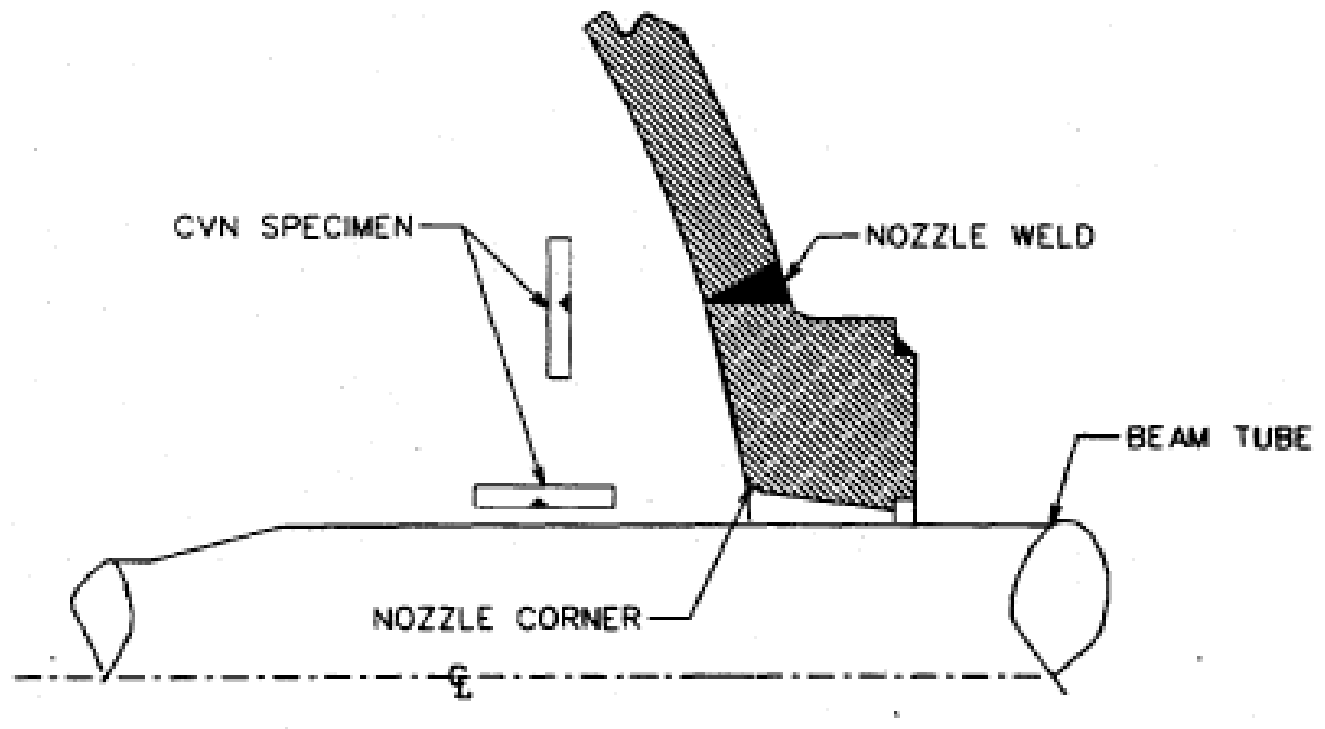

$\underline{\mathrm{HB}-2}$

Fig. 5. Schematic showing the location and orientation of CVN specimens for HFIR upgraded beam tubes with respect to the nozzle weld and corner.

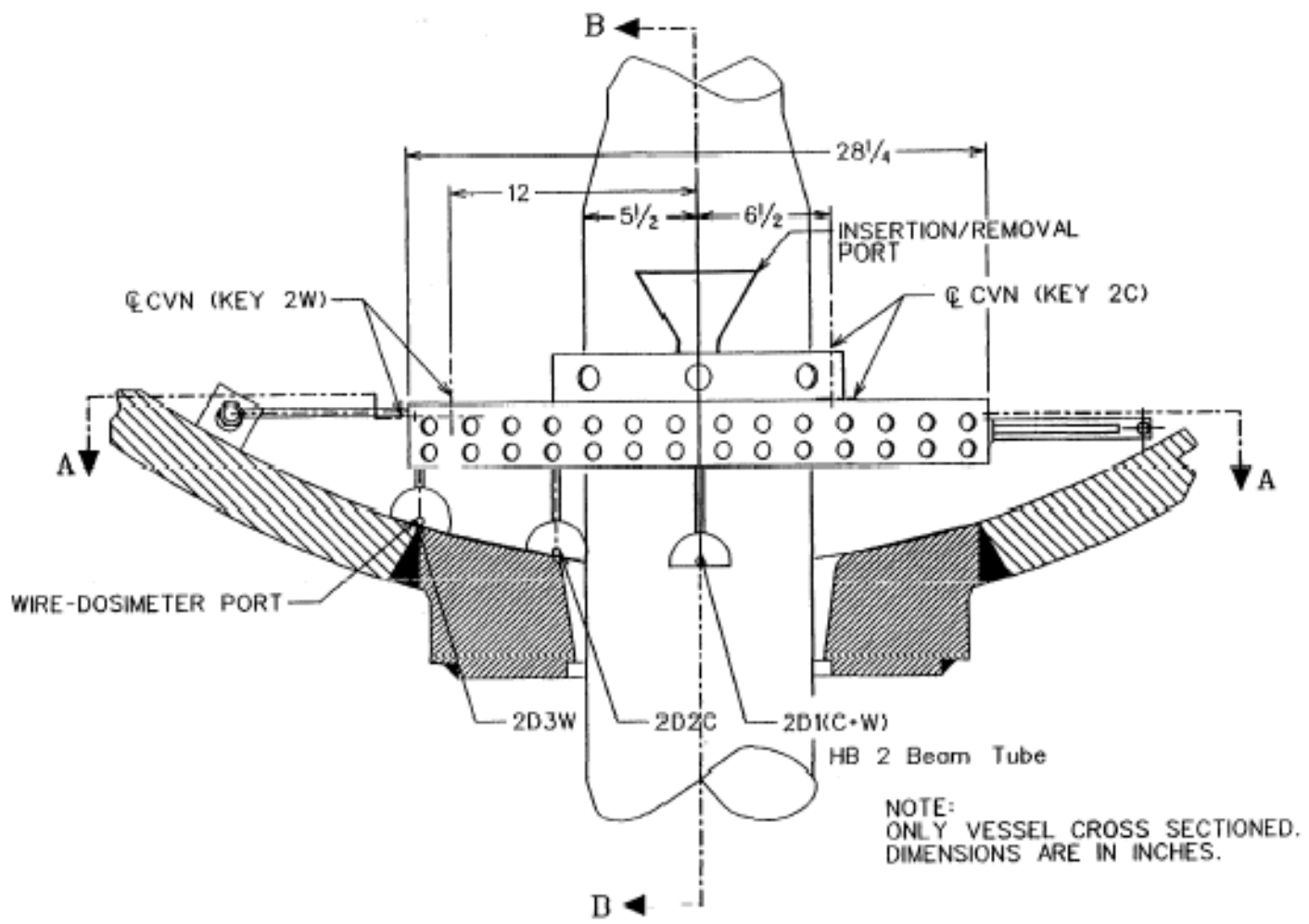

Fig. 6. Schematic of HB-2 surveillance Keys $2 \mathrm{C}$ and $2 \mathrm{~W}$ and the locations of the tubular dosimeters (2D1, 2D2, and 2D3). 


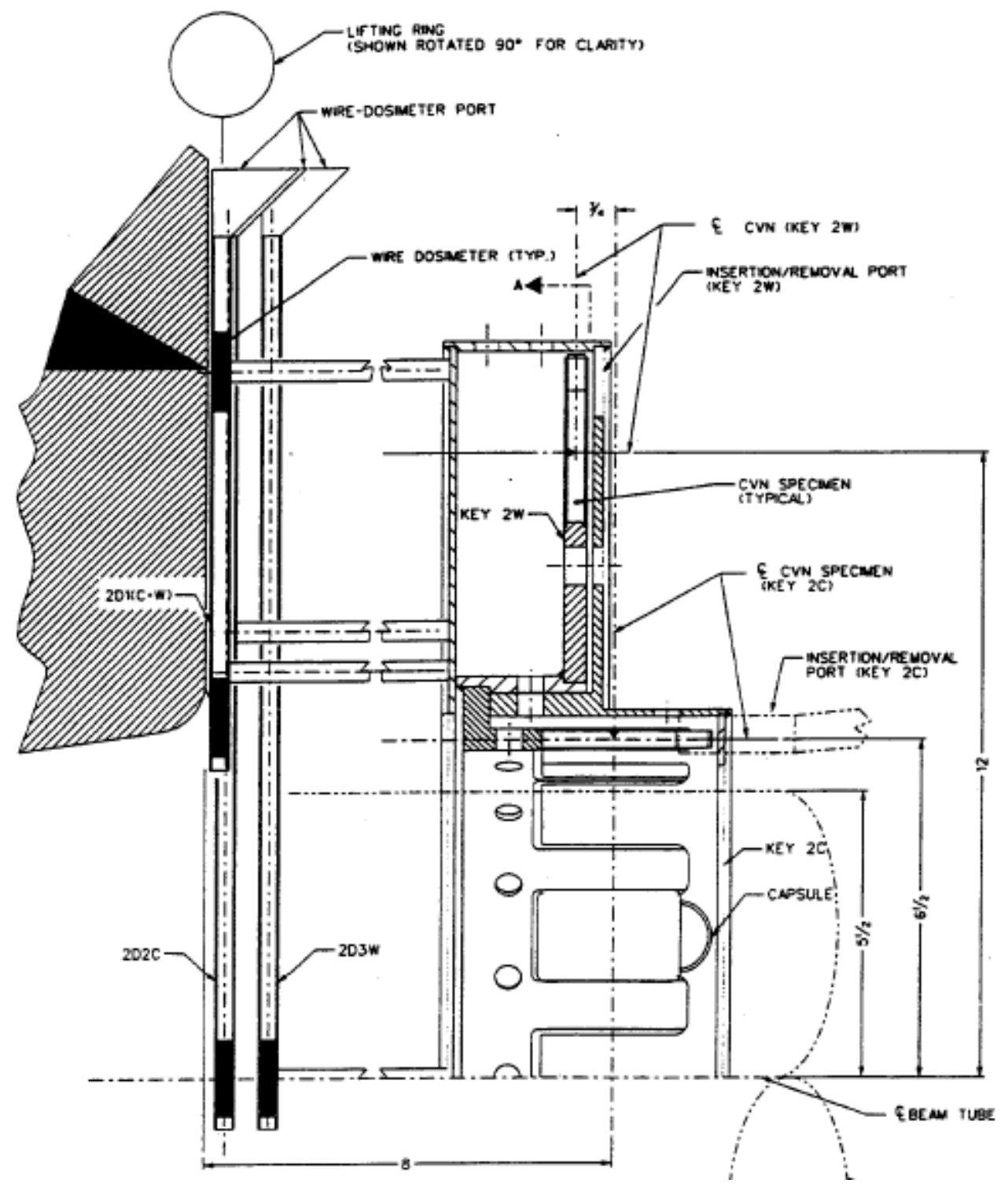

Fig. 7. Vertical cross section of Key 2 showing the location of CVN specimens and tubular dosimeter sets. 


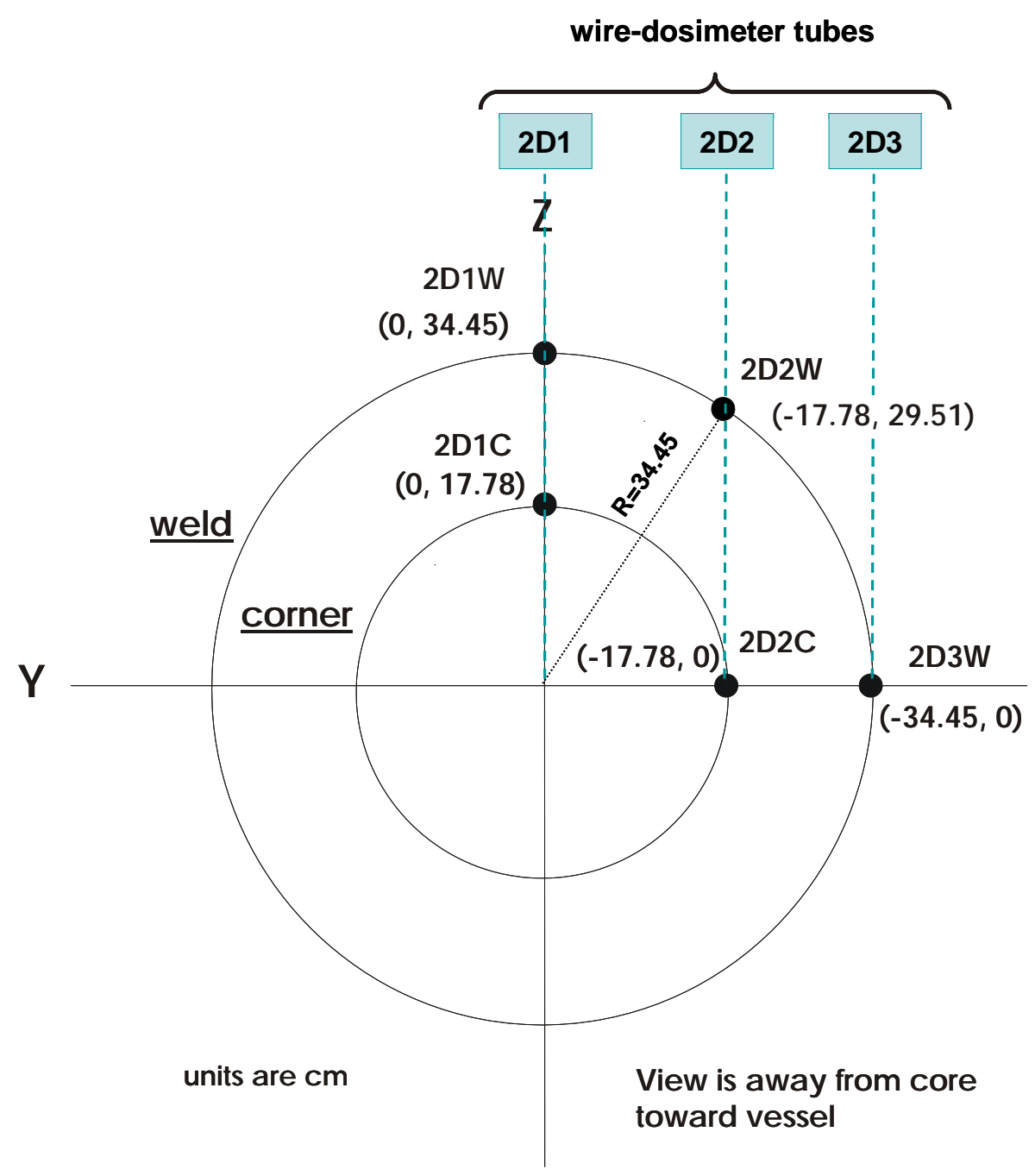

Fig. 8. Locations and labeling of the dosimeter vials in the tubular dosimeter capsules at the HB-2 Key 2D location. The letters "C" or "W" are appended to the key position IDs to indicate dosimeter vial location relative to the nozzle corner $(\mathrm{C})$ or weld $(\mathrm{W})$, respectively. 


\section{THE ANALYSIS}

To obtain the best estimates of the radiation damage parameters from the interpretation and analysis of the physics dosimetry, the spectrum adjustment method, consistent with the American Society of Testing and Materials (ASTM) standard E 944, "Application of Neutron Spectrum Adjustment Methods in Reactor Surveillance," was used. ${ }^{10}$ An extension of the methodology to accommodate both neutrons and gamma rays was developed in the earlier HFIR analysis. ${ }^{11,12}$

The unique aspect of the HFIR radiation field is that at locations away from the beam tubes, the gammaray field intensity is high enough to contribute significantly to the response of dosimeters such as neptunium fission monitors or Be HAFMs, as well as to the radiation damage to the PV material. Therefore, rather than using just neutron flux in determining the irradiation parameters, one needs to consider the gamma-ray flux as well. The methodology for using a coupled neutron-gamma spectrum adjustment procedure, which is suitable for analyzing dosimetry experiments in such mixed fields, was developed in an earlier analysis of the HFIR PV surveillance dosimetry, reported in detail in Ref. 11 and is further described in Ref. 12.

The multigroup neutron and gamma fluxes at the locations of dosimeter capsules were obtained from transport calculations. ${ }^{6,13,14}$ The calculated neutron and gamma fluxes are listed in Appendix B. The LSL-M ${ }^{15}$ computer code package was used to perform neutron and gamma spectrum adjustment calculations and other required data conversions and/or manipulations. The measured specific activities were converted to reaction rates with the ACT program from the LSL-M2 package. Separate runs were performed for cycles 400 and 401, and the reactor core power vs time history was taken into account for each of the two cycles. The neutron reaction cross sections were taken from the Cross-95 cross-section library ${ }^{16}$ and were converted into the group structure used in the transport calculations with the FLXPRO code from the LSL-M2 package. The CALACT code was used to fold the calculated fluxes with the reaction cross sections to obtain the calculated reaction rates. The FLXPRO code was also used to process cross-section covariances and flux covariance information into the group structure used for unfolding. The cross sections for photo-fission reactions were taken from Ref. 17 and for the photoneutron reactions on ${ }^{9} \mathrm{Be}$ from Refs. 18 and 19.

The complete uncertainty analysis of the neutron transport calculations is much more time-consuming and expensive than the transport calculations themselves, and it was not performed for this analysis. Therefore, the HFIR-specific spectrum covariance information was not available, and the approximate neutron and gamma spectrum covariance matrix was prepared as in the previous analysis (Refs. 11 and 12), based in part on data from Ref. 20. For a set of dosimeters considered in a single adjustment run, which included dosimeters irradiated within a single capsule, the standard deviations of the calculated fluxes were selected so that the $\chi 2$ per degree of freedom for the adjustment calculation was approximately 1.00, which indicates that the differences between the measured and calculated reaction rates are consistent with the assigned standard deviations. A $\chi 2$ per degree of freedom smaller than one means that the estimated uncertainties are likely conservative; therefore, in such cases when the flux uncertainties were $\sim 20 \%$, no attempt was made to further decrease the uncertainty of the calculated flux entering the adjustment calculation. This is based on the generally accepted estimate that the uncertainties of the calculated PV fluxes are typically in the range 10-20\% (e.g., see Ref. 21). The standard deviations listed in Tables 4 to 17 for the calculated irradiation parameters are therefore merely reflecting the uncertainties assumed for the calculated fluxes and do not necessarily represent the actual uncertainties of the calculations. 
Uncertainties used for all the "measured" reactions rates considered are given in Table 3 . The values listed represent estimates based on the guidance provided in the relevant ASTM standards (Ref. 22), previous experience, and critical examination of measurements available for this analysis; however, they were not obtained by rigorous propagation of the uncertainties through the measurement process.

Table 3. Uncertainties of the measured reaction rates (as used in the adjustment calculations)

\begin{tabular}{ccl}
\hline Reaction & $\begin{array}{c}\text { Uncertainty } \\
(\%)\end{array}$ & \multicolumn{1}{c}{ Comments } \\
\hline NI58 (N,P) CO58 & 5 & \\
FE54 (N,P) MN54 & 5 & \\
CU63 (N,A) CO60 & 5 & \\
NP237(N,F) & 8 & Np oxide encapsulated in vanadium. \\
BE (N,X) HE4 & 5 & Be monitors encapsulated in stainless steel. \\
CO59 (N,G) CO60 & 7 & $\begin{array}{l}\text { "Diluted" cobalt dosimeter in the form of Co/Al } \\
\text { alloy with 0.100 wt \% Co. }\end{array}$ \\
CO59 (N,G) CO60 (AL) & 5 & $\begin{array}{l}\text { Pure (undiluted) } \\
\text { 0.035-in. thick gadolinium cover. } \\
\text { Diluted cobalt dosimeter in the form of Co/Al alloy } \\
\text { with 0.100 wt \% cobalt under } 0.035 \text {-in. thick } \\
\text { gadolinium cover. }\end{array}$ \\
CO59 (N,G) CO60 (GD) & 8 & \\
CO59 (N,G) CO60 (GD,AL) & 5 & \\
FE58 (N,G) FE59 & 10 &
\end{tabular}

${ }^{a}$ The 1-sigma uncertainty is given. 


\section{RESULTS AND DISCUSSION}

The best-estimate irradiation parameters were obtained by performing least-squares adjustment of the calculated neutron and gamma fluxes and the measured responses of radiometric monitors and $\mathrm{Be}$ HAFMs. In this report, the terms "best-estimate" and "adjusted" are used synonymously with regard to irradiation parameters. All adjusted irradiation parameters represent the cycle-averaged quantities.

For each dosimeter set location, the calculated and the best-estimate irradiation parameters are provided. The irradiation parameters selected are the flux of neutrons with energies above $1 \mathrm{MeV}$, above $0.5 \mathrm{MeV}$, above $0.1 \mathrm{MeV}$, and below $0.41 \mathrm{eV}$, the neutron-induced displacement per atom (dpa) rate in iron calculated with ASTM dpa cross sections [dpa/s (Fe-ASTM)] and with iron dpa cross sections generated from ENDF-B-VI cross-section library [dpa/s (FE-ENDF-VI)], the gamma-ray flux (Gamma Flux), the gamma-ray induced displacement rate per atom in iron (Gamma dpa/s), and the combined neutron and gamma induced atom displacement rate (Neutron + Gamma dpa/s), which is the sum of dpa/s (Fe-ASTM) and gamma-ray induced dpa/s.

The gamma-ray flux, gamma dpa rate, and combined neutron and gamma dpa rates are provided only at locations where the Be HAFMs and the neptunium fission monitors were irradiated. These two monitors are the only ones sensitive to the gamma-ray flux as well as the neutron flux.

A summary of the most important adjusted irradiation parameters is given in Table 4 . The calculated and adjusted irradiation parameters for all locations where the dosimeter sets were irradiated are listed in Tables 5-17. Tables 5-17 also list the ratios of adjusted-to-calculated irradiation parameters. These ratios quantify the changes in the irradiation parameters due to adjustment. The ratio of the measured-tocalculated $(\mathrm{M} / \mathrm{C})$ reaction rate for the Ni-58(n,p) reaction is also given for all the locations and is typically expected to be in reasonable agreement with the adjusted-to-calculated ratio for the neutron flux $(\mathrm{E}>1 \mathrm{MeV})$. All adjusted irradiation parameters provided in this report are for a reactor power of $85 \mathrm{MW}$.

The activities of Ce-141 and Ru-103 often provided Np-237 fission rates inconsistent with Cs-137 and $\mathrm{Zr}-95$ measurements. While the reason for this is not completely understood, it may be related to the relatively long waiting period between the end of irradiation and the start of the measurements. The halflives of Ce-141 and Ru-103 are $32.50 \mathrm{~d}$ and $39.26 \mathrm{~d}$, respectively, and are much shorter than the half-life of Zr-95 (64.02 d) and the half-life of Cs-137 (30.1 years). Therefore, the decay of Ce-141 and Ru-103 during the waiting period was much more severe and may have affected the reliability of the measurements of $\mathrm{Ce}-141$ and $\mathrm{Ru}-103$. For this reason, the $\mathrm{Ce}-141$ and $\mathrm{Ru}-103$ measurements were not used in the adjustment calculations, with the exception of DOS-1, DOS-2, and DOS-6 capsules, where Ru-103 was in better agreement with Cs-137 and Zr-95 and was used in the adjustment.

In the analysis of three capsules, DOS-4, DOS-8, and DOS-9, all the measured activities of the Np-237 fission products were neglected. The reason was that for Cs-137 and Zr-95 the calculated-to-measured reaction rate ratios were significantly different from those of $\mathrm{Ni}$ and $\mathrm{Be}$, and, if included in the adjustment, would lower the adjusted fast neutron flux $(\mathrm{E}>1 \mathrm{MeV})$ and total (neutron + gamma) dpa rate by 4 to $8 \%$. Because of the observed inconsistencies in the measured activities of the Np-237 fission products discussed above and other concerns about the measurements of the neptunium dosimeters, the neptunium dosimeter results were considered less reliable than those of other dosimeters. It was therefore considered preferable not to use neptunium measurements in the adjustment calculations for the capsules listed above, which results in higher adjusted irradiation parameters. Full assessment of the impact of the dosimeters not used in the adjustment is provided in Appendix C. 
At some locations nondiluted and diluted cobalt dosimeters were irradiated together. The ratio of these two activities provides an estimate of the neutron self-shielding correction for the nondiluted dosimeter. Because the diluted cobalt dosimeters do not require such corrections, their activities were considered more reliable. Typically, if the diluted and nondiluted cobalt activities were available for the same location, only the activity of the diluted dosimeter was used in the adjustment calculations.

The reaction rates for the $\mathrm{Cu}-63(\mathrm{n}, \alpha)$ reaction were found to be significantly underestimated by the calculations at the DOS-4, DOS-8, DOS-9, HRB2-2G and 2A, and HRB2-1G and 1A locations. The exclusion of the $\mathrm{Cu}-63(\mathrm{n}, \alpha) \mathrm{Co}-60$ reaction from the adjustment calculations at these locations improved the consistency of adjustment (observed through reduced $\chi^{2}$ per degree of freedom) and resulted in higher values for adjusted irradiation parameters. In particular, the elimination of copper dosimeters from the adjustment resulted in up to $\sim 5 \%$ higher adjusted fast neutron flux $(\mathrm{E}>1 \mathrm{MeV})$ and up to $\sim 3 \%$ higher adjusted total (neutron + gamma) dpa rate. Details about the effect of eliminating the copper dosimeters from the adjustment are discussed in Appendix C.

While the reason for underestimation of the Co-60 activity in the copper dosimeters was not found, several potential culprits can be suggested. On the measurement side, the possibility exists that Co-59 impurities in the copper could have been converted to Co-60 through thermal neutron absorption because the copper wires were not covered with gadolinium. The best agreement with calculations occurred at locations where the thermal flux was lowest (DOS-6, at Key 7), and the worst agreement was at locations where thermal flux was highest (DOS-8 and DOS-9, at Key 2C). On the computational side, there is a possibility that the maximum energy of the neutrons considered $(14.92 \mathrm{MeV})$ was too low to accurately predict the $\mathrm{Cu}-63(\mathrm{n}, \alpha)$ reaction rate; the six highest energy groups produce more that $98 \%$ of $\mathrm{Cu}-63(\mathrm{n}$, $\alpha$ ) reaction rate. Another possibility would be that the fission spectrum used in the calculations has too low an intensity at the high-energy tail. Yet another possibility would be that streaming of high-energy neutrons through the beam tubes was not accurately predicted by the calculation, given that the problem was most prevalent close to the beam tube. Further investigation of these options was beyond the scope of this work.

The need to eliminate measurements of several copper dosimeters is unusual, in particular because four out of the five locations where copper dosimeters were not used in the adjustment are very close to the beam tubes, and two of them are close to the corner of the nozzle of the beam tube. While excluding these copper dosimeters produced higher irradiation parameters, it would be advisable to try to understand this pattern better in any future dosimetry experiments.

As discussed above, several of the measured reaction rates were not used in the adjustment calculations; a complete list of these reactions is given in Table 18. A detailed assessment of the impact of the excluded reactions is provided in Appendix C.

The locations 2D1C and 2D2C are of particular interest because they correspond to the corner of the nozzle of beam tube 2, which was enlarged. Here, only a short discussion is given; a more detailed analysis is provided in Appendix C. These two locations are in the most intense neutron and gamma-ray field of all the locations where the measurements were performed, except for the Key $2 \mathrm{C}$ locations, which were closer to the beam tube and the reactor core. The transport calculations were performed with a cylindrical R-Z model and consequently predicted identical fluxes at both locations (2D1C, 2D2C), as can be observed from the calculated values in Tables 16 and 17, respectively. However, the adjusted fast neutron flux and the total dpa rate are $\sim 34 \%$ and $\sim 29 \%$ higher, respectively, at the location 2D2C than at the location 2D1C. While this appears to be a big difference, the measured specific activities clearly indicate that the neutron and gamma-ray fields are more intense at $2 \mathrm{D} 2 \mathrm{C}$ than they are at $2 \mathrm{D} 1 \mathrm{C}$.

A similar pattern of increase from the " 12 o'clock" to " 3 o'clock" position is also observed for the nozzle 
weld locations, 2D1W, 2D2W, and 2D3W, which are all at the same radial distance from the center of the beam tube. Therefore, the measurements quite clearly reveal differences in the neutron field at seemingly symmetric locations around beam line 2 . It needs to be pointed out that even slight deviation between the actual locations of the dosimeters during the irradiation and the locations considered in the analysis could cause the differences due to very steep neutron flux gradient. Additional three-dimensional discrete ordinates transport calculations showed less than 5\% variation in neutron induced dpa rate from the 12 o'clock to the 3 o'clock location. ${ }^{23}$ For the thermal-neutron induced dpa rate the calculations showed $\sim 40 \%$ increase from the 12 o'clock to the 3 o'clock location; however, the thermal neutrons contributed only $\sim 10 \%$ to the total dpa rate. The three-dimensional calculations were also performed with the Monte Carlo based computer code, for the neutron energy range above $27 \mathrm{keV}$. The Monte Carlo results were in good agreement with discrete ordinates calculations and again showed very little difference in neutron dpa rate at 12 o'clock to 3 o'clock position. ${ }^{23}$ The three-dimensional calculations therefore did not predict the variations observed in the measurements, which may suggest that the measurements were not performed exactly at the intended locations. Finally, it is also necessary to point out considerable inconsistencies in the measured reaction rates especially at location $2 \mathrm{D} 1 \mathrm{C}$, where there is a sizable disagreement in the calculated-to-measured ratios for the beryllium and neptunium dosimeters, and even between the two nickel dosimeters used at this location. In light of all these observations, it would be desirable to repeat the dosimetry experiments at the locations near the beam line nozzle.

Appendix D documents adjusted irradiation parameters as given in the preliminary report, dated November 18, 2005.

The dosimeter experiments were performed during a full fuel cycle and, consequently, the measured reaction rates and the adjusted irradiation parameters are representative of the cycle-averaged quantities. The neutron fluxes from transport calculations were the averages of the beginning-of-cycle and the endof-cycle fluxes for Keys 2 and 7 and for the "end-of-cycle" conditions for Key 3. The differences in neutron spectrum between the beginning and the end-of-cycle have only small effect on the adjusted irradiation parameters; this is discussed in more detail in Appendix E.

Appendix F summarizes information about the computer codes used for the analysis of the dosimeters and the information on the computer runs performed. 
Table 4. Summary of the adjusted irradiation parameters

\begin{tabular}{|c|c|c|c|c|c|c|c|c|c|c|c|c|c|}
\hline \multirow{2}{*}{$\begin{array}{l}\text { Location } \\
\text { in HFIR }\end{array}$} & \multirow{2}{*}{$\begin{array}{l}\text { Capsule ID and location } \\
\text { within the capsule }\end{array}$} & \multicolumn{3}{|c|}{$\begin{array}{c}\text { Neutron flux } \\
(\mathrm{E}>1 \mathrm{MeV})\end{array}$} & \multicolumn{3}{|c|}{ Neutron dpa/s } & \multicolumn{3}{|c|}{ Gamma dpa/s } & \multicolumn{3}{|c|}{$\begin{array}{c}\text { (Neutron + Gamma) } \\
\text { dpa/s }\end{array}$} \\
\hline & & $\begin{array}{c}\text { Value } \\
\left(\mathrm{cm}^{-2} \mathrm{~s}^{-1}\right)\end{array}$ & \pm & $\begin{array}{l}\text { Std. }^{b} \\
(\%)\end{array}$ & $\begin{array}{c}\text { Value } \\
\left(\mathrm{s}^{-1}\right)\end{array}$ & \pm & $\begin{array}{l}\text { Std. }^{b} \\
(\%)\end{array}$ & $\begin{array}{c}\text { Value } \\
\left(\mathrm{s}^{-1}\right)\end{array}$ & \pm & $\begin{array}{l}\text { Std. }^{b} \\
(\%)\end{array}$ & $\begin{array}{c}\text { Value } \\
\left(\mathrm{s}^{-1}\right)\end{array}$ & \pm & $\begin{array}{l}\text { Std. }^{b} \\
(\%)\end{array}$ \\
\hline $\mathrm{K} 2 \mathrm{~W}, \mathrm{P} 8$ & DOS-1, Pos. 2 & $2.74 \mathrm{E}+09$ & \pm & 7 & 4.10E-12 & \pm & 6 & $2.31 \mathrm{E}-12$ & \pm & 8 & $6.40 \mathrm{E}-12$ & \pm & 5 \\
\hline K2W, P32 & DOS-2, Pos. 2 & $2.94 \mathrm{E}+09$ & \pm & 7 & $4.36 \mathrm{E}-12$ & \pm & 6 & $1.84 \mathrm{E}-12$ & \pm & 8 & $6.20 \mathrm{E}-12$ & \pm & 5 \\
\hline K3, P3 & DOS-3, Pos. 2 & $1.17 \mathrm{E}+09$ & \pm & 15 & $2.15 \mathrm{E}-12$ & \pm & 13 & $1.38 \mathrm{E}-12$ & \pm & 9 & $3.54 \mathrm{E}-12$ & \pm & 9 \\
\hline K3, P8 & DOS-4, Pos. 2 & $2.64 \mathrm{E}+09$ & \pm & 8 & $4.38 \mathrm{E}-12$ & \pm & 7 & $1.05 \mathrm{E}-12$ & \pm & 12 & $5.43 \mathrm{E}-12$ & \pm & 6 \\
\hline K3, P10 & DOS-5, Pos. 2 & $1.80 \mathrm{E}+09$ & \pm & 10 & $3.16 \mathrm{E}-12$ & \pm & 9 & $9.40 \mathrm{E}-13$ & \pm & 9 & $4.10 \mathrm{E}-12$ & \pm & 7 \\
\hline $\mathrm{K} 7, \mathrm{P} 1$ & DOS-6, Pos. 2 & $7.06 \mathrm{E}+07$ & \pm & 6 & $1.14 \mathrm{E}-13$ & \pm & 6 & $9.93 \mathrm{E}-13$ & \pm & 6 & $1.11 \mathrm{E}-12$ & \pm & 5 \\
\hline $\mathrm{K} 2 \mathrm{C}, \mathrm{P} 1$ & DOS-8 & $1.01 \mathrm{E}+11$ & \pm & 6 & $1.73 \mathrm{E}-10$ & \pm & 5 & $9.73 \mathrm{E}-12$ & \pm & 22 & $1.83 \mathrm{E}-10$ & \pm & 5 \\
\hline $\mathrm{K} 2 \mathrm{C}, \mathrm{P} 4$ & DOS-9 & $9.97 \mathrm{E}+10$ & \pm & 9 & $1.69 \mathrm{E}-10$ & \pm & 7 & $1.04 \mathrm{E}-11$ & \pm & 25 & $1.80 \mathrm{E}-10$ & \pm & 6 \\
\hline $2 \mathrm{D} 1 \mathrm{~W}$ & HRB2-1G and 1A, Pos. 4 & $8.97 \mathrm{E}+08$ & \pm & 6 & $1.71 \mathrm{E}-12$ & \pm & 6 & $9.71 \mathrm{E}-13$ & \pm & 7 & $2.68 \mathrm{E}-12$ & \pm & 4 \\
\hline $2 \mathrm{D} 2 \mathrm{~W}$ & HRB2-2G and 2A, Pos. 4 & $9.97 \mathrm{E}+08$ & \pm & 6 & $1.87 \mathrm{E}-12$ & \pm & 6 & $9.98 \mathrm{E}-13$ & \pm & 7 & $2.86 \mathrm{E}-12$ & \pm & 4 \\
\hline $2 \mathrm{D} 3 \mathrm{~W}$ & HRB2-3G and 3A, Pos. 2 & $1.10 \mathrm{E}+09$ & \pm & 6 & $1.99 \mathrm{E}-12$ & \pm & 6 & $1.18 \mathrm{E}-12$ & \pm & 7 & $3.17 \mathrm{E}-12$ & \pm & 4 \\
\hline $2 \mathrm{D} 2 \mathrm{C}$ & HRB2-2G and 2A, Pos. 2 & $2.99 \mathrm{E}+10$ & \pm & 6 & $6.24 \mathrm{E}-11$ & \pm & 4 & $3.23 \mathrm{E}-12$ & \pm & 21 & $6.56 \mathrm{E}-11$ & \pm & 4 \\
\hline $2 \mathrm{D} 1 \mathrm{C}$ & HRB2-1G and 1A, Pos. 2 & $2.23 \mathrm{E}+10$ & \pm & 12 & $4.48 \mathrm{E}-11$ & \pm & 7 & $6.13 \mathrm{E}-12$ & \pm & 31 & $5.09 \mathrm{E}-11$ & \pm & 7 \\
\hline
\end{tabular}

${ }^{a} \mathrm{~K}=$ key, $\mathrm{P}=$ position in key, $\mathrm{W}=$ nozzle weld, $\mathrm{C}=$ nozzle corner, $2 \mathrm{D}=$ tubular dosimeter at $\mathrm{HB}-2$.

${ }^{b}$ Percent standard deviation. 
Table 5. Calculated and adjusted irradiation parameters at K2W, P8 (capsule DOS-1)

\begin{tabular}{|c|c|c|c|c|c|c|c|}
\hline & Irradiation parameter & $\begin{array}{c}\text { Calculated } \\
\text { value } \\
()^{a}\end{array}$ & $\begin{array}{l}\text { Std. }^{b} \\
(\%)\end{array}$ & $\begin{array}{c}\text { Adjusted } \\
\text { value } \\
()^{a}\end{array}$ & $\begin{array}{l}\text { Std. }^{b} \\
(\%)\end{array}$ & $\begin{array}{l}\text { Adj./ } \\
\text { calc. }\end{array}$ & $\begin{array}{c}\operatorname{Ni58(n,p)} \\
M_{M} / C^{c}\end{array}$ \\
\hline \multirow{6}{*}{ Position 1} & Neutron flux $(\mathrm{E}>1 \mathrm{MeV})$ & $2.07 \mathrm{E}+09$ & 19 & $2.04 \mathrm{E}+09$ & 7 & 0.99 & \multirow[t]{6}{*}{1.01} \\
\hline & Neutron flux $(\mathrm{E}>0.5 \mathrm{MeV})$ & $3.07 \mathrm{E}+09$ & 19 & $3.03 \mathrm{E}+09$ & 7 & 0.99 & \\
\hline & Neutron flux $(\mathrm{E}>0.1 \mathrm{MeV})$ & $4.39 \mathrm{E}+09$ & 19 & $4.30 \mathrm{E}+09$ & 8 & 0.98 & \\
\hline & Neutron flux $(\mathrm{E}<0.414 \mathrm{eV})$ & $1.44 \mathrm{E}+10$ & 40 & $1.15 \mathrm{E}+10$ & 6 & 0.80 & \\
\hline & Neutron dpa/s (Fe, ASTM) & $3.13 \mathrm{E}-12$ & 18 & $3.05 \mathrm{E}-12$ & 6 & 0.98 & \\
\hline & Neutron dpa/s (Fe, ENDF-VI) & $3.09 \mathrm{E}-12$ & 18 & $3.02 \mathrm{E}-12$ & 6 & 0.98 & \\
\hline \multirow{9}{*}{ Position 2} & Neutron flux $(\mathrm{E}>1 \mathrm{MeV})$ & $2.36 \mathrm{E}+09$ & 19 & $2.74 \mathrm{E}+09$ & 7 & 1.16 & \multirow[t]{9}{*}{1.20} \\
\hline & Neutron flux $(E>0.5 \mathrm{MeV})$ & $3.52 \mathrm{E}+09$ & 19 & $4.08 \mathrm{E}+09$ & 8 & 1.16 & \\
\hline & Neutron flux $(\mathrm{E}>0.1 \mathrm{MeV})$ & $5.07 \mathrm{E}+09$ & 19 & $5.83 \mathrm{E}+09$ & 8 & 1.15 & \\
\hline & Neutron flux $(\mathrm{E}<0.414 \mathrm{eV})$ & $1.69 \mathrm{E}+10$ & 40 & $1.71 \mathrm{E}+10$ & 22 & 1.01 & \\
\hline & Neutron dpa/s (Fe, ASTM) & $3.57 \mathrm{E}-12$ & 18 & $4.10 \mathrm{E}-12$ & 6 & 1.15 & \\
\hline & Neutron dpa/s (Fe, ENDF-VI) & $3.53 \mathrm{E}-12$ & 18 & $4.05 \mathrm{E}-12$ & 6 & 1.15 & \\
\hline & Gamma dpa/s & $2.00 \mathrm{E}-12$ & 27 & $2.31 \mathrm{E}-12$ & 8 & 1.15 & \\
\hline & Gamma flux & $8.17 \mathrm{E}+12$ & 28 & $9.85 \mathrm{E}+12$ & 16 & 1.21 & \\
\hline & Neutron + gamma dpa/s & $5.57 \mathrm{E}-12$ & & $6.40 \mathrm{E}-12$ & 5 & 1.15 & \\
\hline \multirow{6}{*}{ Position 3} & Neutron flux (E > 1 MeV) & $2.93 \mathrm{E}+09$ & 19 & $3.83 \mathrm{E}+09$ & 7 & 1.31 & \multirow[t]{6}{*}{1.33} \\
\hline & Neutron flux $(\mathrm{E}>0.5 \mathrm{MeV})$ & $4.44 \mathrm{E}+09$ & 19 & $5.80 \mathrm{E}+09$ & 7 & 1.31 & \\
\hline & Neutron flux $(\mathrm{E}>0.1 \mathrm{MeV})$ & $6.46 \mathrm{E}+09$ & 19 & $8.40 \mathrm{E}+09$ & 8 & 1.30 & \\
\hline & Neutron flux $(\mathrm{E}<0.414 \mathrm{eV})$ & $2.26 \mathrm{E}+10$ & 40 & $3.36 \mathrm{E}+10$ & 6 & 1.49 & \\
\hline & Neutron dpa/s (Fe, ASTM) & $4.46 \mathrm{E}-12$ & 18 & $5.85 \mathrm{E}-12$ & 6 & 1.31 & \\
\hline & Neutron dpa/s (Fe, ENDF-VI) & $4.41 \mathrm{E}-12$ & 18 & $5.79 \mathrm{E}-12$ & 6 & 1.31 & \\
\hline
\end{tabular}

${ }^{a}$ Units are $\mathrm{cm}^{-2} \mathrm{~s}^{-1}$ for neutron and gamma-ray fluxes and $\mathrm{s}^{-1}$ for $\mathrm{dpa} / \mathrm{s}$.

${ }^{b}$ Percent standard deviation. The $\chi 2$ per degree of freedom for the adjustment run was 7.683E-01.

${ }^{c}$ Nickel measured/calculated reaction rate ratios for the three vials are shown for reference. 
Table 6. Calculated and adjusted irradiation parameters at K2W, P32 (capsule DOS-2)

\begin{tabular}{|c|c|c|c|c|c|c|c|}
\hline & Irradiation parameter & $\begin{array}{c}\text { Calculated } \\
\text { value } \\
()^{a}\end{array}$ & $\begin{array}{l}\text { Std. }^{b} \\
(\%)\end{array}$ & $\begin{array}{l}\text { Adjusted } \\
\text { value } \\
()^{a}\end{array}$ & $\begin{array}{l}\text { Std. }^{b} \\
(\%)\end{array}$ & $\begin{array}{l}\text { Adj./ } \\
\text { calc. }\end{array}$ & $\begin{array}{c}\text { Ni58(n,p) } \\
\text { M/C }^{\mathrm{c}}\end{array}$ \\
\hline \multirow{6}{*}{ Position 1} & Neutron flux $(\mathrm{E}>1 \mathrm{MeV})$ & $2.07 \mathrm{E}+09$ & 19 & $2.21 \mathrm{E}+09$ & 7 & 1.07 & \multirow[t]{6}{*}{1.08} \\
\hline & Neutron flux $(\mathrm{E}>0.5 \mathrm{MeV})$ & $3.07 \mathrm{E}+09$ & 19 & $3.26 \mathrm{E}+09$ & 7 & 1.06 & \\
\hline & Neutron flux $(\mathrm{E}>0.1 \mathrm{MeV})$ & $4.39 \mathrm{E}+09$ & 19 & $4.63 \mathrm{E}+09$ & 8 & 1.05 & \\
\hline & Neutron flux $(\mathrm{E}<0.414 \mathrm{eV})$ & $1.44 \mathrm{E}+10$ & 40 & $9.75 \mathrm{E}+09$ & 5 & 0.68 & \\
\hline & Neutron dpa/s (Fe, ASTM) & $3.13 \mathrm{E}-12$ & 18 & $3.27 \mathrm{E}-12$ & 6 & 1.05 & \\
\hline & Neutron dpa/s (Fe, ENDF-VI) & $3.09 \mathrm{E}-12$ & 18 & $3.23 \mathrm{E}-12$ & 6 & 1.05 & \\
\hline \multirow{9}{*}{ Position 2} & Neutron flux $(\mathrm{E}>1 \mathrm{MeV})$ & $2.36 \mathrm{E}+09$ & 19 & $2.94 \mathrm{E}+09$ & 7 & 1.25 & \multirow[t]{9}{*}{1.29} \\
\hline & Neutron flux $(\mathrm{E}>0.5 \mathrm{MeV})$ & $3.52 \mathrm{E}+09$ & 19 & $4.37 \mathrm{E}+09$ & 8 & 1.24 & \\
\hline & Neutron flux $(E>0.1 \mathrm{MeV})$ & $5.07 \mathrm{E}+09$ & 19 & $6.23 \mathrm{E}+09$ & 8 & 1.23 & \\
\hline & Neutron flux $(\mathrm{E}<0.414 \mathrm{eV})$ & $1.69 \mathrm{E}+10$ & 40 & $1.46 \mathrm{E}+10$ & 22 & 0.86 & \\
\hline & Neutron dpa/s (Fe, ASTM) & $3.57 \mathrm{E}-12$ & 18 & $4.36 \mathrm{E}-12$ & 6 & 1.22 & \\
\hline & Neutron dpa/s (Fe, ENDF-VI) & $3.53 \mathrm{E}-12$ & 18 & $4.31 \mathrm{E}-12$ & 6 & 1.22 & \\
\hline & Gamma dpa/s & $2.00 \mathrm{E}-12$ & 27 & $1.84 \mathrm{E}-12$ & 8 & 0.92 & \\
\hline & Gamma flux & $8.17 \mathrm{E}+12$ & 28 & $7.86 \mathrm{E}+12$ & 16 & 0.96 & \\
\hline & Neutron + gamma dpa/s & $5.57 \mathrm{E}-12$ & & $6.20 \mathrm{E}-12$ & 5 & 1.11 & \\
\hline \multirow{6}{*}{ Position 3} & Neutron flux $(\mathrm{E}>1 \mathrm{MeV})$ & $2.93 \mathrm{E}+09$ & 19 & $4.00 \mathrm{E}+09$ & 7 & 1.37 & \multirow[t]{6}{*}{1.41} \\
\hline & Neutron flux $(\mathrm{E}>0.5 \mathrm{MeV})$ & $4.44 \mathrm{E}+09$ & 19 & $6.04 \mathrm{E}+09$ & 7 & 1.36 & \\
\hline & Neutron flux $(\mathrm{E}>0.1 \mathrm{MeV})$ & $6.46 \mathrm{E}+09$ & 19 & $8.73 \mathrm{E}+09$ & 8 & 1.35 & \\
\hline & Neutron flux $(\mathrm{E}<0.414 \mathrm{eV})$ & $2.26 \mathrm{E}+10$ & 40 & $2.54 \mathrm{E}+10$ & 5 & 1.12 & \\
\hline & Neutron dpa/s (Fe, ASTM) & $4.46 \mathrm{E}-12$ & 18 & $6.01 \mathrm{E}-12$ & 6 & 1.35 & \\
\hline & Neutron dpa/s (Fe, ENDF-VI) & $4.41 \mathrm{E}-12$ & 18 & $5.95 \mathrm{E}-12$ & 6 & 1.35 & \\
\hline
\end{tabular}

${ }^{a}$ Units are $\mathrm{cm}^{-2} \mathrm{~s}^{-1}$ for neutron and gamma-ray fluxes and $\mathrm{s}^{-1}$ for $\mathrm{dpa} / \mathrm{s}$.

${ }^{b}$ Percent standard deviation. The $\chi 2$ per degree of freedom for the adjustment run was $6.035 \mathrm{E}-01$.

${ }^{c} \mathrm{Nickel}$ measured/calculated reaction rate ratios for the three vials are shown for reference. 
Table 7. Calculated and adjusted irradiation parameters at K3, P3 (capsule DOS-3)

\begin{tabular}{|c|c|c|c|c|c|c|c|}
\hline & Irradiation Parameter & $\begin{array}{c}\text { Calculated } \\
\text { value } \\
()^{a}\end{array}$ & $\begin{array}{l}\text { Std. }^{b} \\
(\%)\end{array}$ & $\begin{array}{c}\text { Adjusted } \\
\text { value } \\
()^{a}\end{array}$ & $\begin{array}{l}\text { Std. }^{b} \\
(\%)\end{array}$ & $\begin{array}{l}\text { Adj./ } \\
\text { calc. }\end{array}$ & $\begin{array}{c}\text { Ni58(n,p) } \\
M_{M} / C^{\mathrm{c}}\end{array}$ \\
\hline \multirow{6}{*}{ Position 1} & Neutron flux $(\mathrm{E}>1 \mathrm{MeV})$ & $9.74 \mathrm{E}+08$ & 58 & $7.29 \mathrm{E}+08$ & 15 & 0.75 & \multirow[t]{6}{*}{0.81} \\
\hline & Neutron flux $(\mathrm{E}>0.5 \mathrm{MeV})$ & $1.68 \mathrm{E}+09$ & 58 & $1.27 \mathrm{E}+09$ & 18 & 0.76 & \\
\hline & Neutron flux $(\mathrm{E}>0.1 \mathrm{MeV})$ & $2.77 \mathrm{E}+09$ & 57 & $2.10 \mathrm{E}+09$ & 21 & 0.76 & \\
\hline & Neutron flux $(\mathrm{E}<0.414 \mathrm{eV})$ & $1.66 \mathrm{E}+10$ & 60 & $1.34 \mathrm{E}+10$ & 7 & 0.81 & \\
\hline & Neutron dpa/s (Fe, ASTM) & $1.66 \mathrm{E}-12$ & 52 & $1.27 \mathrm{E}-12$ & 14 & 0.76 & \\
\hline & Neutron dpa/s (Fe, ENDF-VI) & $1.65 \mathrm{E}-12$ & 52 & $1.26 \mathrm{E}-12$ & 14 & 0.76 & \\
\hline \multirow{9}{*}{ Position 2} & Neutron flux $(\mathrm{E}>1 \mathrm{MeV})$ & $1.32 \mathrm{E}+09$ & 58 & $1.17 \mathrm{E}+09$ & 15 & 0.89 & \multirow[t]{9}{*}{0.95} \\
\hline & Neutron flux $(\mathrm{E}>0.5 \mathrm{MeV})$ & $2.27 \mathrm{E}+09$ & 58 & $2.05 \mathrm{E}+09$ & 17 & 0.90 & \\
\hline & Neutron flux $(\mathrm{E}>0.1 \mathrm{MeV})$ & $3.75 \mathrm{E}+09$ & 57 & $3.40 \mathrm{E}+09$ & 20 & 0.91 & \\
\hline & Neutron flux $(\mathrm{E}<0.414 \mathrm{eV})$ & $3.07 \mathrm{E}+10$ & 60 & $3.39 \mathrm{E}+10$ & 38 & 1.10 & \\
\hline & Neutron dpa/s (Fe, ASTM) & $2.31 \mathrm{E}-12$ & 50 & $2.15 \mathrm{E}-12$ & 13 & 0.93 & \\
\hline & Neutron dpa/s (Fe, ENDF-VI) & $2.29 \mathrm{E}-12$ & 51 & $2.13 \mathrm{E}-12$ & 13 & 0.93 & \\
\hline & Gamma dpa/s & $9.83 \mathrm{E}-13$ & 54 & $1.38 \mathrm{E}-12$ & 9 & 1.41 & \\
\hline & Gamma flux & $3.25 \mathrm{E}+12$ & 56 & $4.69 \mathrm{E}+12$ & 28 & 1.44 & \\
\hline & Neutron + gamma dpa/s & $3.29 \mathrm{E}-12$ & & $3.54 \mathrm{E}-12$ & 9 & 1.07 & \\
\hline \multirow{6}{*}{ Position 3} & Neutron flux $(\mathrm{E}>1 \mathrm{MeV})$ & $2.04 \mathrm{E}+09$ & 59 & $1.82 \mathrm{E}+09$ & 15 & 0.89 & \multirow[t]{6}{*}{0.97} \\
\hline & Neutron flux $(\mathrm{E}>0.5 \mathrm{MeV})$ & $3.54 \mathrm{E}+09$ & 58 & $3.22 \mathrm{E}+09$ & 18 & 0.91 & \\
\hline & Neutron flux $(\mathrm{E}>0.1 \mathrm{MeV})$ & $5.88 \mathrm{E}+09$ & 57 & $5.45 \mathrm{E}+09$ & 20 & 0.93 & \\
\hline & Neutron flux $(\mathrm{E}<0.414 \mathrm{eV})$ & $7.10 \mathrm{E}+10$ & 60 & $1.05 \mathrm{E}+11$ & 6 & 1.47 & \\
\hline & Neutron dpa/s (Fe, ASTM) & $3.78 \mathrm{E}-12$ & 49 & $3.86 \mathrm{E}-12$ & 13 & 1.02 & \\
\hline & Neutron dpa/s (Fe, ENDF-VI) & $3.74 \mathrm{E}-12$ & 49 & $3.79 \mathrm{E}-12$ & 13 & 1.02 & \\
\hline
\end{tabular}

${ }^{a}$ Units are $\mathrm{cm}^{-2} \mathrm{~s}^{-1}$ for neutron and gamma-ray fluxes and $\mathrm{s}^{-1}$ for $\mathrm{dpa} / \mathrm{s}$.

${ }^{b}$ Percent standard deviation. The $\chi 2$ per degree of freedom for the adjustment run was 8.677E-01.

${ }^{c}$ Nickel measured/calculated reaction rate ratios for the three vials are shown for reference. 
Table 8. Calculated and adjusted irradiation parameters at K3, P8 (capsule DOS-4)

\begin{tabular}{|c|c|c|c|c|c|c|c|}
\hline & Irradiation parameter & $\begin{array}{c}\text { Calculated } \\
\text { value } \\
()^{a}\end{array}$ & $\begin{array}{l}\text { Std. }^{b} \\
(\%)\end{array}$ & $\begin{array}{c}\text { Adjusted } \\
\text { value } \\
()^{a}\end{array}$ & $\begin{array}{l}\text { Std. }^{b} \\
(\%)\end{array}$ & $\begin{array}{l}\text { Adj./ } \\
\text { calc. }\end{array}$ & $\begin{array}{c}\text { Ni58(n,p) } \\
M_{M} / C^{c}\end{array}$ \\
\hline \multirow{6}{*}{ Position 1} & Neutron flux $(\mathrm{E}>1 \mathrm{MeV})$ & $1.84 \mathrm{E}+09$ & 19 & $1.76 \mathrm{E}+09$ & 7 & 0.96 & \multirow[t]{6}{*}{0.97} \\
\hline & Neutron flux $(\mathrm{E}>0.5 \mathrm{MeV})$ & $2.98 \mathrm{E}+09$ & 19 & $2.85 \mathrm{E}+09$ & 8 & 0.95 & \\
\hline & Neutron flux $(E>0.1 \mathrm{MeV})$ & $4.74 \mathrm{E}+09$ & 19 & $4.48 \mathrm{E}+09$ & 8 & 0.94 & \\
\hline & Neutron flux $(\mathrm{E}<0.414 \mathrm{eV})$ & $3.15 \mathrm{E}+10$ & 40 & $1.94 \mathrm{E}+10$ & 6 & 0.62 & \\
\hline & Neutron dpa/s (Fe, ASTM) & $3.13 \mathrm{E}-12$ & 18 & $2.86 \mathrm{E}-12$ & 6 & 0.92 & \\
\hline & Neutron dpa/s (Fe, ENDF-VI) & $3.10 \mathrm{E}-12$ & 18 & $2.84 \mathrm{E}-12$ & 6 & 0.92 & \\
\hline \multirow{9}{*}{ Position 2} & Neutron flux $(\mathrm{E}>1 \mathrm{MeV})$ & $2.32 \mathrm{E}+09$ & 19 & $2.64 \mathrm{E}+09$ & 8 & 1.14 & \multirow[t]{9}{*}{1.16} \\
\hline & Neutron flux $(\mathrm{E}>0.5 \mathrm{MeV})$ & $3.91 \mathrm{E}+09$ & 19 & $4.45 \mathrm{E}+09$ & 8 & 1.14 & \\
\hline & Neutron flux $(\mathrm{E}>0.1 \mathrm{MeV})$ & $6.39 \mathrm{E}+09$ & 19 & $7.19 \mathrm{E}+09$ & 9 & 1.13 & \\
\hline & Neutron flux $(\mathrm{E}<0.414 \mathrm{eV})$ & $4.26 \mathrm{E}+10$ & 40 & $3.62 \mathrm{E}+10$ & 22 & 0.85 & \\
\hline & Neutron dpa/s (Fe, ASTM) & $3.99 \mathrm{E}-12$ & 18 & $4.38 \mathrm{E}-12$ & 7 & 1.10 & \\
\hline & Neutron dpa/s (Fe, ENDF-VI) & $3.95 \mathrm{E}-12$ & 18 & $4.35 \mathrm{E}-12$ & 7 & 1.10 & \\
\hline & Gamma dpa/s & $9.97 \mathrm{E}-13$ & 27 & $1.05 \mathrm{E}-12$ & 12 & 1.06 & \\
\hline & Gamma flux & $3.16 \mathrm{E}+12$ & 28 & $3.33 \mathrm{E}+12$ & 18 & 1.05 & \\
\hline & Neutron + gamma dpa/s & $4.98 \mathrm{E}-12$ & & $5.43 \mathrm{E}-12$ & 6 & 1.09 & \\
\hline \multirow{6}{*}{ Position 3} & Neutron flux $(\mathrm{E}>1 \mathrm{MeV})$ & $3.20 \mathrm{E}+09$ & 19 & $4.00 \mathrm{E}+09$ & 7 & 1.25 & \multirow[t]{6}{*}{1.27} \\
\hline & Neutron flux $(\mathrm{E}>0.5 \mathrm{MeV})$ & $5.39 \mathrm{E}+09$ & 19 & $6.72 \mathrm{E}+09$ & 8 & 1.25 & \\
\hline & Neutron flux $(\mathrm{E}>0.1 \mathrm{MeV})$ & $8.81 \mathrm{E}+09$ & 19 & $1.09 \mathrm{E}+10$ & 8 & 1.24 & \\
\hline & Neutron flux $(\mathrm{E}<0.414 \mathrm{eV})$ & $9.43 \mathrm{E}+10$ & 40 & $1.13 \mathrm{E}+11$ & 6 & 1.20 & \\
\hline & Neutron dpa/s (Fe, ASTM) & $5.80 \mathrm{E}-12$ & 18 & $7.15 \mathrm{E}-12$ & 6 & 1.23 & \\
\hline & Neutron dpa/s (Fe, ENDF-VI) & $5.73 \mathrm{E}-12$ & 18 & $7.06 \mathrm{E}-12$ & 6 & 1.23 & \\
\hline
\end{tabular}

${ }^{a}$ Units are $\mathrm{cm}^{-2} \mathrm{~s}^{-1}$ for neutron and gamma-ray fluxes and $\mathrm{s}^{-1}$ for $\mathrm{dpa} / \mathrm{s}$.

${ }^{b}$ Percent standard deviation. The $\chi 2$ per degree of freedom for the adjustment run was 5.724E-01.

${ }^{c}$ Nickel measured/calculated reaction rate ratios for the three vials are shown for reference. 
Table 9. Calculated and adjusted irradiation parameters at K3, P10 (capsule DOS-5)

\begin{tabular}{|c|c|c|c|c|c|c|c|}
\hline & Irradiation parameter & $\begin{array}{c}\text { Calculated } \\
\text { value } \\
()^{a}\end{array}$ & $\begin{array}{l}\text { Std. }^{b} \\
(\%)\end{array}$ & $\begin{array}{l}\text { Adjusted } \\
\text { value } \\
()^{a}\end{array}$ & $\begin{array}{l}\text { Std. }^{b} \\
(\%)\end{array}$ & $\begin{array}{l}\text { Adj./ } \\
\text { calc. }\end{array}$ & $\begin{array}{c}\text { Ni58(n,p) } \\
M_{M} / C^{c}\end{array}$ \\
\hline \multirow{6}{*}{ Position 1} & Neutron flux $(\mathrm{E}>1 \mathrm{MeV})$ & $1.16 \mathrm{E}+09$ & 34 & $1.13 \mathrm{E}+09$ & 10 & 0.98 & \multirow[t]{6}{*}{1.06} \\
\hline & Neutron flux $(\mathrm{E}>0.5 \mathrm{MeV})$ & $1.98 \mathrm{E}+09$ & 34 & $1.93 \mathrm{E}+09$ & 11 & 0.97 & \\
\hline & Neutron flux $(E>0.1 \mathrm{MeV})$ & $3.25 \mathrm{E}+09$ & 33 & $3.16 \mathrm{E}+09$ & 13 & 0.97 & \\
\hline & Neutron flux $(\mathrm{E}<0.414 \mathrm{eV})$ & $1.67 \mathrm{E}+10$ & 40 & $2.09 \mathrm{E}+10$ & 5 & 1.25 & \\
\hline & Neutron dpa/s (Fe, ASTM) & $1.97 \mathrm{E}-12$ & 30 & $1.98 \mathrm{E}-12$ & 9 & 1.00 & \\
\hline & Neutron dpa/s (Fe, ENDF-VI) & $1.96 \mathrm{E}-12$ & 31 & $1.96 \mathrm{E}-12$ & 9 & 1.00 & \\
\hline \multirow{9}{*}{ Position 2} & Neutron flux $(\mathrm{E}>1 \mathrm{MeV})$ & $1.72 \mathrm{E}+09$ & 34 & $1.80 \mathrm{E}+09$ & 10 & 1.05 & \multirow[t]{9}{*}{1.14} \\
\hline & Neutron flux $(\mathrm{E}>0.5 \mathrm{MeV})$ & $2.98 \mathrm{E}+09$ & 34 & $3.11 \mathrm{E}+09$ & 11 & 1.04 & \\
\hline & Neutron flux $(E>0.1 \mathrm{MeV})$ & $4.95 \mathrm{E}+09$ & 33 & $5.11 \mathrm{E}+09$ & 12 & 1.03 & \\
\hline & Neutron flux $(\mathrm{E}<0.414 \mathrm{eV})$ & $3.23 \mathrm{E}+10$ & 40 & $3.72 \mathrm{E}+10$ & 24 & 1.15 & \\
\hline & Neutron dpa/s (Fe, ASTM) & $3.00 \mathrm{E}-12$ & 30 & $3.16 \mathrm{E}-12$ & 9 & 1.05 & \\
\hline & Neutron dpa/s (Fe, ENDF-VI) & $2.98 \mathrm{E}-12$ & 30 & $3.14 \mathrm{E}-12$ & 9 & 1.05 & \\
\hline & Gamma dpa/s & $9.87 \mathrm{E}-13$ & 31 & $9.40 \mathrm{E}-13$ & 9 & 0.95 & \\
\hline & Gamma flux & $3.47 \mathrm{E}+12$ & 33 & $3.56 \mathrm{E}+12$ & 19 & 1.02 & \\
\hline & Neutron + gamma dpa/s & $3.99 \mathrm{E}-12$ & & $4.10 \mathrm{E}-12$ & 7 & 1.03 & \\
\hline \multirow{6}{*}{ Position 3} & Neutron flux $(\mathrm{E}>1 \mathrm{MeV})$ & $2.36 \mathrm{E}+09$ & 34 & $2.97 \mathrm{E}+09$ & 10 & 1.26 & \multirow[t]{6}{*}{1.33} \\
\hline & Neutron flux $(\mathrm{E}>0.5 \mathrm{MeV})$ & $4.08 \mathrm{E}+09$ & 34 & $5.13 \mathrm{E}+09$ & 11 & 1.26 & \\
\hline & Neutron flux $(E>0.1 \mathrm{MeV})$ & $6.79 \mathrm{E}+09$ & 33 & $8.52 \mathrm{E}+09$ & 13 & 1.26 & \\
\hline & Neutron flux $(\mathrm{E}<0.414 \mathrm{eV})$ & $6.73 \mathrm{E}+10$ & 40 & $1.07 \mathrm{E}+11$ & 4 & 1.59 & \\
\hline & Neutron dpa/s (Fe, ASTM) & $4.29 \mathrm{E}-12$ & 29 & $5.63 \mathrm{E}-12$ & 8 & 1.31 & \\
\hline & Neutron dpa/s (Fe, ENDF-VI) & 4.24E-12 & 29 & $5.55 \mathrm{E}-12$ & 9 & 1.31 & \\
\hline
\end{tabular}

${ }^{a}$ Units are $\mathrm{cm}^{-2} \mathrm{~s}^{-1}$ for neutron and gamma-ray fluxes and $\mathrm{s}^{-1}$ for $\mathrm{dpa} / \mathrm{s}$.

${ }^{b}$ Percent standard deviation. The $\chi 2$ per degree of freedom for the adjustment run was 5.561E-01.

${ }^{c}$ Nickel measured/calculated reaction rate ratios for the three vials are shown for reference. 
Table 10. Calculated and adjusted irradiation parameters at K7, P1 (capsule DOS-6)

\begin{tabular}{|c|c|c|c|c|c|c|c|}
\hline & Irradiation parameter & $\begin{array}{c}\text { Calculated } \\
\text { value } \\
()^{\mathrm{a}}\end{array}$ & $\begin{array}{l}\text { Std. }^{b} \\
(\%)\end{array}$ & $\begin{array}{c}\text { Adjusted } \\
\text { value } \\
()^{a}\end{array}$ & $\begin{array}{l}\text { Std. }^{b} \\
(\%)\end{array}$ & $\begin{array}{l}\text { Adj./ } \\
\text { calc. }\end{array}$ & $\begin{array}{c}\operatorname{Ni58(n,p)} \\
M_{M} / C^{c}\end{array}$ \\
\hline \multirow{6}{*}{ Position 1} & Neutron flux $(\mathrm{E}>1 \mathrm{MeV})$ & $5.48 \mathrm{E}+07$ & 19 & $6.90 \mathrm{E}+07$ & 5 & 1.26 & 1.27 \\
\hline & FLUENCE $>0.5 \mathrm{MEV}$ & $6.49 \mathrm{E}+07$ & 19 & $8.14 \mathrm{E}+07$ & 6 & 1.25 & \\
\hline & FLUENCE $>0.1 \mathrm{MEV}$ & $7.82 \mathrm{E}+07$ & 19 & $9.77 \mathrm{E}+07$ & 6 & 1.25 & \\
\hline & FLUENCE $>0.414$ & $2.43 \mathrm{E}+08$ & 40 & $1.30 \mathrm{E}+08$ & 4 & 0.53 & \\
\hline & Neutron dpa/s (FE, ASTM) & 8.93E-14 & 18 & $1.11 \mathrm{E}-13$ & 4 & 1.25 & \\
\hline & Neutron dpa/s (FE, ENDF-VI) & $8.71 \mathrm{E}-14$ & 18 & $1.09 \mathrm{E}-13$ & 4 & 1.25 & \\
\hline \multirow{9}{*}{ Position 2} & Neutron flux $(\mathrm{E}>1 \mathrm{MeV})$ & $5.48 \mathrm{E}+07$ & 19 & $7.06 \mathrm{E}+07$ & 6 & 1.29 & 1.30 \\
\hline & Neutron flux $(\mathrm{E}>0.5 \mathrm{MeV})$ & $6.49 \mathrm{E}+07$ & 19 & $8.33 \mathrm{E}+07$ & 7 & 1.28 & \\
\hline & Neutron flux $(\mathrm{E}>0.1 \mathrm{MeV})$ & $7.82 \mathrm{E}+07$ & 19 & $1.00 \mathrm{E}+08$ & 7 & 1.28 & \\
\hline & Neutron flux $(\mathrm{E}<0.414 \mathrm{eV})$ & $2.43 \mathrm{E}+08$ & 40 & $1.43 \mathrm{E}+08$ & 21 & 0.59 & \\
\hline & Neutron dpa/s (Fe, ASTM) & 8.93E-14 & 18 & $1.14 \mathrm{E}-13$ & 6 & 1.28 & \\
\hline & Neutron dpa/s (Fe, ENDF-VI) & $8.71 \mathrm{E}-14$ & 18 & $1.11 \mathrm{E}-13$ & 6 & 1.28 & \\
\hline & Gamma dpa/s & $9.81 \mathrm{E}-13$ & 27 & $9.93 \mathrm{E}-13$ & 6 & 1.01 & \\
\hline & Gamma flux & $7.07 \mathrm{E}+12$ & 29 & $7.58 \mathrm{E}+12$ & 16 & 1.07 & \\
\hline & Neutron + gamma dpa/s & $1.07 \mathrm{E}-12$ & & $1.11 \mathrm{E}-12$ & 5 & 1.03 & \\
\hline \multirow{6}{*}{ Position 3} & Neutron flux $(\mathrm{E}>1 \mathrm{MeV})$ & $5.48 \mathrm{E}+07$ & 19 & $6.80 \mathrm{E}+07$ & 5 & 1.24 & 1.27 \\
\hline & Neutron flux $(\mathrm{E}>0.5 \mathrm{MeV})$ & $6.49 \mathrm{E}+07$ & 19 & $8.03 \mathrm{E}+07$ & 6 & 1.24 & \\
\hline & Neutron flux $(E>0.1 \mathrm{MeV})$ & $7.82 \mathrm{E}+07$ & 19 & $9.62 \mathrm{E}+07$ & 6 & 1.23 & \\
\hline & Neutron flux $(\mathrm{E}<0.414 \mathrm{eV})$ & $2.43 \mathrm{E}+08$ & 40 & $1.14 \mathrm{E}+08$ & 4 & 0.47 & \\
\hline & Neutron dpa/s (Fe, ASTM) & $8.93 \mathrm{E}-14$ & 18 & $1.10 \mathrm{E}-13$ & 4 & 1.23 & \\
\hline & Neutron dpa/s (Fe, ENDF-VI) & $8.71 \mathrm{E}-14$ & 18 & $1.07 \mathrm{E}-13$ & 4 & 1.23 & \\
\hline
\end{tabular}

${ }^{a}$ Units are $\mathrm{cm}^{-2} \mathrm{~s}^{-1}$ for neutron and gamma-ray fluxes and $\mathrm{s}^{-1}$ for $\mathrm{dpa} / \mathrm{s}$.

${ }^{b}$ Percent standard deviation. The $\chi 2$ per degree of freedom for the adjustment run was 9.608E-01

${ }^{c}$ Nickel measured/calculated reaction rate ratios for the three vials are shown for reference. 
Table 11. Calculated and adjusted irradiation parameters at K2C, P1 (capsule DOS-8)

\begin{tabular}{|c|c|c|c|c|c|c|}
\hline Irradiation parameter $^{a}$ & $\begin{array}{c}\text { Calculated } \\
\text { value } \\
()^{b}\end{array}$ & $\begin{array}{l}\text { Std. } \\
(\%)\end{array}$ & $\begin{array}{c}\text { Adjusted } \\
\text { value } \\
()^{b}\end{array}$ & $\begin{array}{l}\text { Std. }{ }^{c} \\
\text { (\%) }\end{array}$ & $\begin{array}{l}\text { Adj./ } \\
\text { calc. }\end{array}$ & $\begin{array}{c}\underset{M}{\operatorname{Ni58(n,p)}} \\
C^{d}\end{array}$ \\
\hline Neutron flux $(\mathrm{E}>1 \mathrm{MeV})$ & $6.72 \mathrm{E}+10$ & 20 & $1.01 \mathrm{E}+11$ & 6 & 1.51 & 1.51 \\
\hline Neutron flux $(\mathrm{E}>0.5 \mathrm{MeV})$ & $1.11 \mathrm{E}+11$ & 19 & $1.66 \mathrm{E}+11$ & 7 & 1.50 & 1.55 \\
\hline Neutron flux $(\mathrm{E}>0.1 \mathrm{MeV})$ & $1.83 \mathrm{E}+11$ & 19 & $2.71 \mathrm{E}+11$ & 8 & 1.48 & 1.51 \\
\hline Neutron flux $(\mathrm{E}<0.414 \mathrm{eV})$ & $2.75 \mathrm{E}+12$ & 20 & $2.60 \mathrm{E}+12$ & 5 & 0.95 & \\
\hline Neutron dpa/s (Fe, ASTM) & $1.25 \mathrm{E}-10$ & 16 & $1.73 \mathrm{E}-10$ & 5 & 1.38 & \\
\hline Neutron dpa/s (Fe, ENDF-VI) & $1.23 \mathrm{E}-10$ & 16 & $1.71 \mathrm{E}-10$ & 5 & 1.39 & \\
\hline Gamma dpa/s & $7.81 \mathrm{E}-12$ & 27 & $9.73 \mathrm{E}-12$ & 22 & 1.25 & \\
\hline Gamma flux & $1.53 \mathrm{E}+13$ & 28 & $1.90 \mathrm{E}+13$ & 24 & 1.24 & \\
\hline Neutron + gamma dpa/s & $1.33 \mathrm{E}-10$ & & $1.83 \mathrm{E}-10$ & 5 & 1.37 & \\
\hline
\end{tabular}

${ }^{a}$ The measurements at the three vial positions were treated together in the adjustment because there was essentially no difference in the measured values, indicating a uniform neutron field throughout the capsule.

${ }^{b}$ Units are $\mathrm{cm}^{-2} \mathrm{~s}^{-1}$ for neutron and gamma-ray fluxes and $\mathrm{s}^{-1}$ for $\mathrm{dpa} / \mathrm{s}$.

${ }^{c}$ Percent standard deviation. The $\chi^{2}$ per degree of freedom for the adjustment run was $4.928 \mathrm{E}-01$.

${ }^{d}$ Nickel measured/calculated reaction rate ratios for the three vials are shown for reference.

Table 12. Calculated and adjusted irradiation parameters at K2C, P4 (capsule DOS-9)

\begin{tabular}{|c|c|c|c|c|c|c|}
\hline Irradiation parameter $^{a}$ & $\begin{array}{c}\text { Calculated } \\
\text { value } \\
()^{b} \\
\end{array}$ & $\begin{array}{l}\text { Std. }^{c} \\
(\%)\end{array}$ & $\begin{array}{c}\text { Adjusted } \\
\text { value } \\
()^{b} \\
\end{array}$ & $\begin{array}{l}\text { Std. } \\
(\%)\end{array}$ & $\begin{array}{l}\text { Adj./ } \\
\text { calc. }\end{array}$ & $\begin{array}{c}\operatorname{Ni58(n,p)} \\
M / C^{d}\end{array}$ \\
\hline Neutron flux $(E>1 \mathrm{MeV})$ & $6.72 \mathrm{E}+10$ & 29 & $9.97 \mathrm{E}+10$ & 9 & 1.48 & 1.44 \\
\hline Neutron flux $(\mathrm{E}>0.5 \mathrm{MeV})$ & $1.11 \mathrm{E}+11$ & 29 & $1.63 \mathrm{E}+11$ & 10 & 1.47 & 1.56 \\
\hline Neutron flux $(\mathrm{E}>0.1 \mathrm{MeV})$ & $1.83 \mathrm{E}+11$ & 29 & $2.62 \mathrm{E}+11$ & 11 & 1.43 & 1.53 \\
\hline Neutron flux $(\mathrm{E}<0.414 \mathrm{eV})$ & $2.75 \mathrm{E}+12$ & 30 & $2.89 \mathrm{E}+12$ & 3 & 1.05 & \\
\hline Neutron dpa/s (Fe, ASTM) & $1.25 \mathrm{E}-10$ & 24 & $1.69 \mathrm{E}-10$ & 7 & 1.35 & \\
\hline Neutron dpa/s (Fe, ENDF-VI) & $1.23 \mathrm{E}-10$ & 24 & $1.67 \mathrm{E}-10$ & 7 & 1.35 & \\
\hline Gamma dpa/s & $7.81 \mathrm{E}-12$ & 36 & $1.04 \mathrm{E}-11$ & 25 & 1.34 & \\
\hline Gamma flux & $1.53 \mathrm{E}+13$ & 37 & $1.99 \mathrm{E}+13$ & 30 & 1.30 & \\
\hline Neutron + gamma dpa/s & $1.33 \mathrm{E}-10$ & & $1.80 \mathrm{E}-10$ & 6 & 1.35 & \\
\hline
\end{tabular}

${ }^{a}$ The measurements at the three vial positions were treated together in the adjustment because there was essentially no difference in the measured values, indicating a uniform neutron field throughout the capsule.

${ }^{b}$ Units are $\mathrm{cm}^{-2} \mathrm{~s}^{-1}$ for neutron and gamma-ray fluxes and $\mathrm{s}^{-1}$ for $\mathrm{dpa} / \mathrm{s}$.

${ }^{c}$ Percent standard deviation. The $\chi^{2}$ per degree of freedom for the adjustment run was 9.625E-01.

${ }^{d}$ Nickel measured/calculated reaction rate ratios for the three vials are shown for reference. 
Table 13. Calculated and adjusted irradiation parameters at 2D1W (capsules HRB2-1G and HRB2-1A, position 4; dosimeter sets GD-15 and AL-28; HRB2-1W)

\begin{tabular}{|c|c|c|c|c|c|c|}
\hline Irradiation parameter $^{a}$ & $\begin{array}{c}\text { Calculated } \\
\text { value } \\
()^{b}\end{array}$ & $\begin{array}{l}\text { Std. }^{c} \\
(\%)\end{array}$ & $\begin{array}{c}\text { Adjusted } \\
\text { value } \\
()^{b}\end{array}$ & $\begin{array}{l}\text { Std. }^{c} \\
(\%)\end{array}$ & $\begin{array}{l}\text { Adj./ } \\
\text { calc. }\end{array}$ & $\begin{array}{c}\text { Ni58(n,p) } \\
\mathbf{M}^{-C^{d}}\end{array}$ \\
\hline Neutron flux $(\mathrm{E}>1 \mathrm{MeV})$ & $9.42 \mathrm{E}+08$ & 19 & $8.97 \mathrm{E}+08$ & 6 & 0.95 & 0.95 \\
\hline Neutron flux $(\mathrm{E}>0.5 \mathrm{MeV})$ & $1.72 \mathrm{E}+09$ & 19 & $1.64 \mathrm{E}+09$ & 7 & 0.96 & 0.93 \\
\hline Neutron flux $(\mathrm{E}>0.1 \mathrm{MeV})$ & $3.01 \mathrm{E}+09$ & 19 & $2.91 \mathrm{E}+09$ & 8 & 0.97 & \\
\hline Neutron flux $(\mathrm{E}<0.414 \mathrm{eV})$ & $1.09 \mathrm{E}+10$ & 40 & $1.61 \mathrm{E}+10$ & 6 & 1.48 & \\
\hline Neutron dpa/s (Fe, ASTM) & $1.71 \mathrm{E}-12$ & 18 & $1.71 \mathrm{E}-12$ & 6 & 1.00 & \\
\hline Neutron dpa/s (Fe, ENDF-VI) & $1.71 \mathrm{E}-12$ & 18 & $1.70 \mathrm{E}-12$ & 6 & 0.99 & \\
\hline Gamma dpa/s & $1.01 \mathrm{E}-12$ & 27 & $9.71 \mathrm{E}-13$ & 7 & 0.96 & \\
\hline Gamma flux & $4.64 \mathrm{E}+12$ & 28 & $4.11 \mathrm{E}+12$ & 16 & 0.89 & \\
\hline Neutron + gamma dpa/s & $2.72 \mathrm{E}-12$ & & $2.68 \mathrm{E}-12$ & 4 & 0.98 & \\
\hline
\end{tabular}

${ }^{a}$ The measurements from cycles 400 and 401 were treated together in the adjustment.

${ }^{b}$ Units are $\mathrm{cm}^{-2} \mathrm{~s}^{-1}$ for neutron and gamma-ray fluxes and $\mathrm{s}^{-1}$ for $\mathrm{dpa} / \mathrm{s}$.

${ }^{c}$ Percent standard deviation. The $\chi 2$ per degree of freedom for the adjustment run was $7.225 \mathrm{E}-01$.

${ }^{d}$ Nickel measured/calculated reaction rate ratios for cycles 400 and 401 are shown for reference.

Table 14. Calculated and adjusted irradiation parameters at 2D2W (capsules HRB2-2G and HRB2-2A, position 4; dosimeter sets GD-17 and AL-30; HRB2-2W)

\begin{tabular}{|c|c|c|c|c|c|c|}
\hline Irradiation parameter $^{a}$ & $\begin{array}{c}\text { Calculated } \\
\text { value } \\
()^{b}\end{array}$ & $\begin{array}{l}\text { Std. }^{c} \\
(\%)\end{array}$ & $\begin{array}{c}\text { Adjusted } \\
\text { value } \\
()^{b}\end{array}$ & $\begin{array}{l}\text { Std. } \\
(\%)\end{array}$ & $\begin{array}{l}\text { Adj./ } \\
\text { calc. }\end{array}$ & $\begin{array}{c}\underset{M}{\operatorname{Ni58(n,p)}} \mathbf{C}^{\mathrm{d}} \\
.\end{array}$ \\
\hline Neutron flux $(\mathrm{E}>1 \mathrm{MeV})$ & $9.42 \mathrm{E}+08$ & 19 & $9.97 \mathrm{E}+08$ & 6 & 1.06 & 1.06 \\
\hline Neutron flux $(\mathrm{E}>0.5 \mathrm{MeV})$ & $1.72 \mathrm{E}+09$ & 19 & $1.82 \mathrm{E}+09$ & 7 & 1.06 & 1.07 \\
\hline Neutron flux $(\mathrm{E}>0.1 \mathrm{MeV})$ & $3.01 \mathrm{E}+09$ & 19 & $3.19 \mathrm{E}+09$ & 8 & 1.06 & \\
\hline Neutron flux $(\mathrm{E}<0.414 \mathrm{eV})$ & $1.09 \mathrm{E}+10$ & 40 & $1.68 \mathrm{E}+10$ & 6 & 1.54 & \\
\hline $\mathrm{dpa} / \mathrm{s}(\mathrm{Fe}, \mathrm{ASTM})$ & $1.71 \mathrm{E}-12$ & 18 & $1.87 \mathrm{E}-12$ & 6 & 1.09 & \\
\hline $\mathrm{dpa} / \mathrm{s} \quad(\mathrm{Fe}, \mathrm{ENDF}-\mathrm{VI})$ & $1.71 \mathrm{E}-12$ & 18 & $1.86 \mathrm{E}-12$ & 6 & 1.09 & \\
\hline Gamma dpa/s & $1.01 \mathrm{E}-12$ & 27 & $9.98 \mathrm{E}-13$ & 7 & 0.99 & \\
\hline Gamma flux & $4.64 \mathrm{E}+12$ & 28 & $4.61 \mathrm{E}+12$ & 16 & 0.99 & \\
\hline Neutron + gamma dpa/s & $2.72 \mathrm{E}-12$ & & $2.86 \mathrm{E}-12$ & 4 & 1.05 & \\
\hline
\end{tabular}


Table 15. Calculated and adjusted irradiation parameters at 2D3W (capsules HRB2-3G and HRB2-3A, position 2; dosimeter sets GD-18 and AL-31; HRB2-3W)

\begin{tabular}{|c|c|c|c|c|c|c|}
\hline Irradiation parameter $^{a}$ & $\begin{array}{c}\text { Calculated } \\
\text { value } \\
()^{b}\end{array}$ & $\begin{array}{l}\text { Std. }^{c} \\
(\%)\end{array}$ & $\begin{array}{l}\text { Adjusted } \\
\text { value } \\
()^{b}\end{array}$ & $\begin{array}{l}\text { Std. }^{c} \\
(\%)\end{array}$ & $\begin{array}{l}\text { Adj./ } \\
\text { calc. }\end{array}$ & $\begin{array}{c}\text { Ni58(n,p) } \\
M_{M} / C^{d}\end{array}$ \\
\hline Neutron flux $(\mathrm{E}>1 \mathrm{MeV})$ & $9.42 \mathrm{E}+08$ & 19 & $1.10 \mathrm{E}+09$ & 6 & 1.16 & 1.22 \\
\hline Neutron flux $(\mathrm{E}>0.5 \mathrm{MeV})$ & $1.72 \mathrm{E}+09$ & 19 & $1.99 \mathrm{E}+09$ & 7 & 1.16 & 1.18 \\
\hline Neutron flux $(E>0.1 \mathrm{MeV})$ & $3.01 \mathrm{E}+09$ & 19 & $3.47 \mathrm{E}+09$ & 8 & 1.15 & \\
\hline Neutron flux $(\mathrm{E}<0.414 \mathrm{eV})$ & $1.09 \mathrm{E}+10$ & 40 & $1.39 \mathrm{E}+10$ & 6 & 1.27 & \\
\hline Neutron dpa/s (Fe, ASTM) & $1.71 \mathrm{E}-12$ & 18 & $1.99 \mathrm{E}-12$ & 6 & 1.16 & \\
\hline Neutron dpa/s (Fe, ENDF-VI) & $1.71 \mathrm{E}-12$ & 18 & $1.99 \mathrm{E}-12$ & 6 & 1.16 & \\
\hline Gamma dpa/s & $1.01 \mathrm{E}-12$ & 27 & $1.18 \mathrm{E}-12$ & 7 & 1.17 & \\
\hline Gamma flux & $4.64 \mathrm{E}+12$ & 28 & $5.33 \mathrm{E}+12$ & 16 & 1.15 & \\
\hline Neutron + gamma dpa/s & $2.72 \mathrm{E}-12$ & & $3.17 \mathrm{E}-12$ & 4 & 1.16 & \\
\hline
\end{tabular}

${ }^{a}$ The measurements from cycles 400 and 401 were treated together in the adjustment.

${ }^{b}$ Units are $\mathrm{cm}^{-2} \mathrm{~s}^{-1}$ for neutron and gamma-ray fluxes and $\mathrm{s}^{-1}$ for $\mathrm{dpa} / \mathrm{s}$.

${ }^{c}$ Percent standard deviation. The $\chi 2$ per degree of freedom for the adjustment run was $4.559 \mathrm{E}-01$.

${ }^{d}$ Nickel measured/calculated reaction rate ratios for cycles 400 and 401 are shown for reference.

Table 16. Calculated and adjusted irradiation parameters at 2D2C (capsules HRB2-2G and HRB2-2A, position 2; dosimeter sets GD-16 and AL-29; HRB2-2C)

\begin{tabular}{|c|c|c|c|c|c|c|}
\hline Irradiation parameter $^{a}$ & $\begin{array}{c}\text { Calculated } \\
\text { value } \\
()^{b}\end{array}$ & $\begin{array}{l}\text { Std. }^{c} \\
(\%)\end{array}$ & $\begin{array}{c}\text { Adjusted } \\
\text { value } \\
()^{b}\end{array}$ & $\begin{array}{l}\text { Std. }{ }^{c} \\
(\%)\end{array}$ & $\begin{array}{l}\text { Adj./ } \\
\text { calc. }\end{array}$ & $\begin{array}{c}\text { Ni58(n,p) } \\
\text { M/C }^{d}\end{array}$ \\
\hline Neutron flux $(\mathrm{E}>1 \mathrm{MeV})$ & $2.79 \mathrm{E}+10$ & 19 & $2.99 \mathrm{E}+10$ & 6 & 1.07 & 1.11 \\
\hline Neutron flux $(E>0.5 \mathrm{MeV})$ & $4.40 \mathrm{E}+10$ & 19 & $4.70 \mathrm{E}+10$ & 6 & 1.07 & 1.08 \\
\hline Neutron flux $(\mathrm{E}>0.1 \mathrm{MeV})$ & $6.95 \mathrm{E}+10$ & 19 & $7.40 \mathrm{E}+10$ & 7 & 1.07 & \\
\hline Neutron flux $(\mathrm{E}<0.414 \mathrm{eV})$ & $2.25 \mathrm{E}+12$ & 20 & $2.08 \mathrm{E}+12$ & 6 & 0.92 & \\
\hline Neutron dpa/s (Fe, ASTM) & $6.10 \mathrm{E}-11$ & 15 & $6.24 \mathrm{E}-11$ & 4 & 1.02 & \\
\hline Neutron dpa/s (Fe, ENDF-VI) & $5.92 \mathrm{E}-11$ & 15 & $6.07 \mathrm{E}-11$ & 4 & 1.02 & \\
\hline Gamma dpa/s & $2.76 \mathrm{E}-12$ & 27 & $3.23 \mathrm{E}-12$ & 21 & 1.17 & \\
\hline Gamma flux & $9.39 \mathrm{E}+12$ & 28 & $1.09 \mathrm{E}+13$ & 24 & 1.16 & \\
\hline Neutron + gamma dpa/s & $6.38 \mathrm{E}-11$ & & $6.56 \mathrm{E}-11$ & 4 & 1.03 & \\
\hline
\end{tabular}

${ }^{a}$ The measurements from cycles 400 and 401 were treated together in the adjustment.

${ }^{b}$ Units are $\mathrm{cm}^{-2} \mathrm{~s}^{-1}$ for neutron and gamma-ray fluxes and $\mathrm{s}^{-1}$ for $\mathrm{dpa} / \mathrm{s}$.

${ }^{c}$ Percent standard deviation. The $\chi 2$ per degree of freedom for the adjustment run was 4.071E-01.

${ }^{d}$ Nickel measured/calculated reaction rate ratios for cycles 400 and 401 are shown for reference. 
Table 17. Calculated and adjusted irradiation parameters at 2D1C (capsules HRB2-1G and HRB2-1A, position 2; dosimeter sets GD-14 and AL-27; HRB2-1C)

\begin{tabular}{|c|c|c|c|c|c|c|}
\hline Irradiation parameter $^{a}$ & $\begin{array}{c}\text { Calculated } \\
\text { value } \\
()^{b}\end{array}$ & $\begin{array}{l}\text { Std. } \\
(\%)\end{array}$ & $\begin{array}{l}\text { Adjusted } \\
\text { value } \\
()^{b}\end{array}$ & $\begin{array}{l}\text { Std. }^{c} \\
(\%)\end{array}$ & $\begin{array}{l}\text { Adj./ } \\
\text { calc. }\end{array}$ & $\begin{array}{c}\underset{M}{\operatorname{Ni58(n,p)}} \\
M_{\mathbf{C}} \mathbf{C}^{\mathrm{d}}\end{array}$ \\
\hline Neutron flux $(\mathrm{E}>1 \mathrm{MeV})$ & $2.79 \mathrm{E}+10$ & 63 & $2.23 \mathrm{E}+10$ & 12 & 0.80 & 0.96 \\
\hline Neutron flux $(\mathrm{E}>0.5 \mathrm{MeV})$ & $4.40 \mathrm{E}+10$ & 63 & $3.41 \mathrm{E}+10$ & 13 & 0.78 & 0.89 \\
\hline Neutron flux $(\mathrm{E}>0.1 \mathrm{MeV})$ & $6.95 \mathrm{E}+10$ & 62 & $5.18 \mathrm{E}+10$ & 15 & 0.75 & \\
\hline Neutron flux $(\mathrm{E}<0.414 \mathrm{eV})$ & $2.25 \mathrm{E}+12$ & 65 & $1.43 \mathrm{E}+12$ & 6 & 0.64 & \\
\hline Neutron dpa/s (Fe, ASTM) & $6.10 \mathrm{E}-11$ & 48 & $4.48 \mathrm{E}-11$ & 7 & 0.73 & \\
\hline Neutron dpa/s (Fe, ENDF-VI) & $5.92 \mathrm{E}-11$ & 49 & $4.35 \mathrm{E}-11$ & 7 & 0.73 & \\
\hline Gamma dpa/s & $2.76 \mathrm{E}-12$ & 58 & $6.13 \mathrm{E}-12$ & 31 & 2.22 & \\
\hline Gamma flux & $9.39 \mathrm{E}+12$ & 61 & $2.10 \mathrm{E}+13$ & 44 & 2.23 & \\
\hline Neutron + gamma dpa/s & $6.38 \mathrm{E}-11$ & & $5.09 \mathrm{E}-11$ & 7 & 0.80 & \\
\hline
\end{tabular}

${ }^{a}$ The measurements from cycles 400 and 401 were treated together in the adjustment.

${ }^{b}$ Units are $\mathrm{cm}^{-2} \mathrm{~s}^{-1}$ for neutron and gamma-ray fluxes and $\mathrm{s}^{-1}$ for $\mathrm{dpa} / \mathrm{s}$.

${ }^{c}$ Percent standard deviation. The $\chi 2$ per degree of freedom for the adjustment run was $1.033 \mathrm{E}+00$.

${ }^{d}$ Nickel measured/calculated reaction rate ratios for cycles 400 and 401 are shown for reference. 
Table 18. Measured activities not used in adjustment

\begin{tabular}{cll}
\hline Location in HFIR & Capsule ID & Measured activities not used in final adjustment runs \\
\hline K2W, P8 & DOS-1, Pos. 2 & NP237(N,F) CE141 GD \\
K2W, P32 & DOS-2, Pos. 1, 2 and 3 & NP237(N,F) CE141 GD and 3 nondiluted Co \\
K3, P3 ${ }^{a}$ & DOS-3, Pos. 2 & NP237(N,F) RU103 GD \\
K3, P8 & 2 Cu dosimeter, 3 (all) Np \\
K3, P10 & DOS-4, Pos. 1, 2 and 3 & NP237(N,F) RU103 GD \\
K7, P1 ${ }^{a}$ & DOS-5, Pos. 2 & none \\
K2C, P1 & DOS-6 & 2 Cu, 4 (all) Np, 1 Co undiluted \\
K2C, P4 & DOS-8 & 2 Cu, 4 (all) Np \\
2D1W ${ }^{a}$ & DOS-9 & NP237(N,F) RU103 GD \\
2D2W ${ }^{a}$ & HRB2-1G and 1A, Pos. 4 & NP237(N,F) RU103 GD \\
2D3W ${ }^{a}$ & HRB2-2G and 2A, Pos. 4 & NP237(N,F) RU103 GD \\
2D2C & HRB2-3G and 3A, Pos. 2 & 1 (all) Cu, 2 Np (CE141, RU103), 1 nondiluted Co \\
2D1C & HRB2-2G and 2A, Pos. 2 & 1 (all) Cu, 2 Np (CE141, RU103), 2 nondiluted Co \\
\hline${ }^{a}$ Indicates locations where the Ce-141 activity was not measured. Note that the Ce-141 activity was not used in the adjustment at all, and \\
the Ru-103 was used only at DOS-1, DOS-2, and DOS-6.
\end{tabular}





\section{CONCLUSION}

This report provides best-estimate irradiation parameters at several locations close to the inner wall of the HFIR pressure vessel and around the beam tubes. The best-estimate irradiation parameters were obtained by performing least-squares adjustment of the calculated neutron and gamma fluxes and the measured responses of radiometric monitors and Be HAFMs. The adjustment method used is consistent with ASTM standard E 944. The analysis revealed considerable inconsistencies in the measured reactions rates at some locations, and because of this several measurements were not used in the adjustment. The effects of the omitted measurements on the adjusted irradiation parameters were carefully investigated. However, it

is nevertheless felt that it would be beneficial to repeat the dosimetry experiments at the locations near the beam line nozzle. Another improvement desirable in the future analyses is determination of the flux covariance matrices via uncertainty analysis of the transport calculations. 



\section{REFERENCES}

1. J. R. McWherter, R. E. Schappel, and J. R. McGuffey, HFIR Pressure Vessel and Structural Components Material Surveillance Program, ORNL/TM-1372, Oak Ridge National Laboratory, Oak Ridge, Tenn., January 1966.

2. R. D. Cheverton, J. G. Merkle, and R. K. Nanstad, Evaluation of HFIR Pressure-Vessel Integrity Considering Radiation Embrittlement, ORNL/TM-10444, Oak Ridge National Laboratory, Oak Ridge, Tenn., April 1988.

3. I. Remec, J. A. Wang, F. B. K. Kam, and K. Farrell, "Effects of Gamma-Induced Displacements on HFIR Pressure Vessel Materials," J. Nucl. Mat. 217, 258-268 (1994).

4. R. D. Cheverton and T. L. Dickson, HFIR Vessel Life Extension with Enlarged HB-2 and HB-4 Beam Tubes, ORNL/TM-13698, Oak Ridge National Laboratory, Oak Ridge, Tenn., December 1998.

5. R. D. Cheverton, An Evaluation of Life Extension of the HFIR Pressure Vessel, ORNL/TM-12877, Oak Ridge National Laboratory, Oak Ridge, Tenn., December 1994.

6. R. D. Cheverton, R. K. Nanstad, and E. D. Blakeman, HFIR Pressure Vessel and Structural Components Materials Surveillance Program, Supplement 2, ORNL/TM1372/S2, Oak Ridge National Laboratory, August 1999.

7. C. A. Baldwin, Assembly/Manufacturing and Installation Plan for HFIR Beam Tube Upgrade Pressure Vessel Surveillance Dosimetry (Experiment Special Procedure, Document No. MET-FE-ESP-25), September 2000.

8. D. C. Glasgow, Dosimeter Counting Narrative and Results in Support of the HFIR Pressure Vessel Life Extension Program, Letter to J. R. Inger, November 11, 2005.

9. B. M. Oliver, Helium Analyses of HFIR Beryllium Dosimeters, work performed under ORNL Purchase Order 4000033417; private communication from Brian M. Oliver, Pacific Northwest National Laboratory, to J. Inger, Oak Ridge National Laboratory, January 3, 2005.

10. ASTM Standard Guide for Application of Neutron Spectrum Adjustment Methods in Reactor Surveillance, E 944, Annual Book of ASTM Standards, Sect. 12, Vol. 12.02, "Nuclear, Solar, and Geothermal Energy."

11. I. Remec and F. B. K. Kam, Neutron Spectra at Different High Flux Isotope Reactor (HFIR) Pressure Vessel Surveillance Locations, NUREG/CR-6117, ORNL/TM-12484, Oak Ridge National Laboratory, Oak Ridge, Tenn., December 1993.

12. I. Remec, Simultaneous Neutron and Gamma Spectrum Adjustment, Proc. Ninth International Symposium on Reactor Dosimetry, Prague, Czech Republic, September 1996, pp. 253-260 (1998).

13. E. D. Blakeman, Neutron and Gamma Fluxes and dpa Rates for the HFIR Vessel Beltline Region (Present and Upgrade Designs), ORNL/TM-13693, Oak Ridge National Laboratory, Oak Ridge, Tenn., November 2000.

14. E. D. Blakeman, Neutron and Gamma Fluxes and dpa Rates for HFIR Vessel Beltline Region (Present and Upgrade Designs) Supplement 1, ORNL/TM-13693/S1, Oak Ridge National Laboratory, Oak Ridge, Tenn., December 2000.

15. F. W. Stallmann, LSL-M2: A Computer Program for Least Squares Logarithmic Adjustment of Neutron Spectra, NUREG/CR-4349, ORNL/TM-9933, Oak Ridge National Laboratory, Oak Ridge, Tenn., March 1986.

16. I. Remec, An Update of the Dosimetry Cross-Section Data Base for the LSL-M2 Adjustment Code, ORNL/TM-13409, Oak Ridge National Laboratory, Oak Ridge, Tenn., October 2004.

17. V. V. Verbinski et al., Nucl. Sci. and Eng. 75, 159-166 (1980). 
18. S. I. Warshaw, " $4 \pi$ Photoneutron Cross-Section for $9 \mathrm{Be}$ from Threshold to $16 \mathrm{MeV}$ : Initial Evaluation," Physical Data Note PD-175, Lawrence Livermore National Laboratory, 1989.

19. E. P. Blizard et al., Reactor Handbook, Vol. III, Part B, "Shielding," p. 23.

20. R. E. Maerker et al., Revision and Extension of the Data Base in the LEPRICON Dosimetry Methodology, EPRI NP-3841, Electric Power Research Institute, Palo Alto, Calif., January 1985.

21. ASTM Standard Guide for Application of Neutron Transport Methods for Reactor Vessel Surveillance, E 482, Annual Book of ASTM Standards, Sect. 12, Vol. 12.02, "Nuclear, Solar, and Geothermal Energy."

22. Annual Book of ASTM Standards, Sect. 12, Vol. 12.02, "Nuclear, Solar, and Geothermal Energy."

23. E. D. Blakeman, Calculation of dpa Rates at the HFIR HB-2 Beam Tube Nozzle Corner Using Three-Dimensional Discrete Ordinates and Monte Carlo Models, ORNL/TM2003/213, Oak Ridge National Laboratory, Oak Ridge, Tenn., June 2004. 
APPENDIX A

MEASURED SPECIFIC ACTIVITIES AND HELIUM CONCENTRATIONS 

Table A.1. Measured specific activities of the dosimeters irradiated during HFIR fuel cycle 400

\begin{tabular}{|c|c|c|c|c|c|}
\hline $\begin{array}{l}\text { Dosimeter } \\
\text { capsule }^{a}\end{array}$ & Reaction & Cover & $\begin{array}{l}\text { Specific } \\
\text { activity } \\
\text { (Bq/mg) }\end{array}$ & Vial ID & $\begin{array}{c}\text { Dosimeter } \\
\text { material }\end{array}$ \\
\hline \multicolumn{6}{|l|}{ DOS1-1 } \\
\hline & NI58 (N,P) CO58 & & $4.66 \mathrm{E}+02$ & AL-01 & Nickel \\
\hline & FE54 (N,P) MN54 & & $7.62 \mathrm{E}+00$ & AL-01 & Iron \\
\hline & FE58 (N,G) FE59 & & $9.67 \mathrm{E}+01$ & & \\
\hline & CU63 (N,A) CO60 & & $1.63 \mathrm{E}-01$ & AL-01 & Copper \\
\hline & $\operatorname{CO} 59(\mathrm{~N}, \mathrm{G}) \mathrm{CO} 60$ & & $2.37 \mathrm{E}+04$ & AL-01 & Cobalt \\
\hline
\end{tabular}

DOS1-2

$\begin{array}{lllll}\text { NI58 (N,P) CO58 } & \text { GD } & 6.05 \mathrm{E}+02 & \text { GD-01 } & \text { Nickel } \\ \text { NP237(N,F) ZR95 } & \text { GD } & 5.27 \mathrm{E}+02 & \text { GD-01 } & \text { Np-237 } \\ \text { NP237(N,F) RU103 } & \text { GD } & 6.60 \mathrm{E}+02 & & \\ \text { NP237(N,F) CS137 } & \text { GD } & 3.05 \mathrm{E}+00 & & \\ \text { NP237(N,F) CE141 } & \text { GD } & 9.67 \mathrm{E}+02 & & \\ \text { CO59 (N,G) CO60 } & \text { GD } & 1.30 \mathrm{E}+03 & \text { GD-01 } & \text { Cobalt }\end{array}$

DOS1-3

NI58 (N,P) CO58

FE54 (N,P) MN54

FE58 (N,G) FE59

CU63 (N,A) CO60

$\operatorname{CO} 59(\mathrm{~N}, \mathrm{G}) \mathrm{CO} 60$

$\begin{array}{lll}8.20 \mathrm{E}+02 & \text { AL-02 } & \text { Nickel } \\ 1.39 \mathrm{E}+01 & \text { AL-02 } & \text { Iron } \\ 2.92 \mathrm{E}+02 & & \\ 2.57 \mathrm{E}-01 & \text { AL-02 } & \text { Copper } \\ 7.02 \mathrm{E}+04 & \text { AL-02 } & \text { Cobalt }\end{array}$

DOS2-1

DOS2-2

NI58 $(\mathrm{N}, \mathrm{P}) \operatorname{CO} 58$
FE54 (N,P) MN54
FE5 $(\mathrm{N}, \mathrm{G})$ FE5
CU63 $(\mathrm{N}, \mathrm{A})$ CO60
CO59 $(\mathrm{N}, \mathrm{G}) \operatorname{CO} 60$
CO59 $(\mathrm{N}, \mathrm{G}) \operatorname{CO} 60$

$\begin{array}{lll}4.94 \mathrm{E}+02 & \text { AL-03 } & \text { Nickel } \\ 8.34 \mathrm{E}+00 & \text { AL-03 } & \text { Iron } \\ 8.62 \mathrm{E}+01 & & \\ 1.81 \mathrm{E}-01 & \text { AL-03 } & \text { Copper } \\ 2.73 \mathrm{E}+01 & \text { AL-03 } & \text { Co/Al } \\ 2.08 \mathrm{E}+04 & \text { AL-03 } & \text { Cobalt }\end{array}$

$\begin{array}{lllll}\text { NI58 (N,P) CO58 } & \text { GD } & 6.52 \mathrm{E}+02 & \text { GD-02 } & \text { Nickel } \\ \text { NP237(N,F) ZR95 } & \text { GD } & 4.56 \mathrm{E}+02 & \text { GD-02 } & \text { Np-237 } \\ \text { NP237(N,F) RU103 } & \text { GD } & 5.75 \mathrm{E}+02 & & \\ \text { NP237(N,F) CS137 } & \text { GD } & 3.11 \mathrm{E}+00 & & \\ \text { NP237(N,F) CE141 } & \text { GD } & 9.27 \mathrm{E}+02 & & \\ \text { CO59 (N,G) CO60 } & \text { GD } & 3.01 \mathrm{E}+00 & \text { GD-02 } & \text { Co/Al } \\ \text { CO59 (N,G) CO60 } & \text { GD } & 1.39 \mathrm{E}+03 & \text { GD-02 } & \text { Cobalt }\end{array}$


Table A.1. (continued)

\begin{tabular}{|c|c|c|c|c|c|}
\hline $\begin{array}{c}\text { Dosimeter } \\
\text { capsule }\end{array}$ & Reaction & Cover & $\begin{array}{l}\text { Specific } \\
\text { activity } \\
\text { (Bq/mg) }\end{array}$ & Vial ID & $\begin{array}{c}\text { Dosimeter } \\
\text { material }\end{array}$ \\
\hline \multicolumn{6}{|l|}{ DOS2-3 } \\
\hline & NI58 (N,P) CO58 & & $8.69 \mathrm{E}+02$ & AL-04 & Nickel \\
\hline & FE54 (N,P) MN54 & & $1.46 \mathrm{E}+01$ & AL-04 & Iron \\
\hline & FE58 (N,G) FE59 & & $2.33 \mathrm{E}+02$ & & \\
\hline & CU63 (N,A) CO60 & & $2.67 \mathrm{E}-01$ & AL-04 & Copper \\
\hline & $\operatorname{CO} 59(\mathrm{~N}, \mathrm{G}) \mathrm{CO} 60$ & & $7.09 \mathrm{E}+01$ & AL-04 & $\mathrm{Co} / \mathrm{Al}$ \\
\hline & $\operatorname{CO} 59(\mathrm{~N}, \mathrm{G}) \operatorname{CO} 60$ & & $5.41 \mathrm{E}+04$ & AL-04 & Cobalt \\
\hline
\end{tabular}

DOS3-1

NI58 (N,P) CO58

FE54 (N,P) MN54

FE58 $(\mathrm{N}, \mathrm{G})$ FE59

CU63 (N,A) CO60

$\mathrm{CO} 59(\mathrm{~N}, \mathrm{G}) \mathrm{CO} 60$

Dos3-2

NI58 (N,P) CO58

NP237(N,F) ZR95

NP237(N,F) RU103

NP237(N,F) CS137

$\mathrm{CO} 59(\mathrm{~N}, \mathrm{G}) \mathrm{CO} 60$

DOS3-3

NI58 (N,P) CO58

FE54 (N,P) MN54

FE58 $(\mathrm{N}, \mathrm{G})$ FE59

CU63 (N,A) CO60

$\mathrm{CO} 59(\mathrm{~N}, \mathrm{G}) \mathrm{CO} 60$

DOS4-1

NI58 (N,P) CO58

FE54 (N,P) MN54

FE58 (N,G) FE59

CU63 (N,A) CO60

$\mathrm{CO} 59(\mathrm{~N}, \mathrm{G}) \mathrm{CO} 60$

DOS4-2

NI58 (N,P) CO58

NP237(N,F) ZR95

NP237(N,F) RU103

NP237(N,F) CS137

$\mathrm{CO} 59(\mathrm{~N}, \mathrm{G}) \mathrm{CO} 60$

\begin{tabular}{|c|c|c|}
\hline $1.19 \mathrm{E}+02$ & AL-05 & Nickel \\
\hline $1.82 \mathrm{E}+00$ & AL-05 & Iron \\
\hline $1.12 \mathrm{E}+02$ & & \\
\hline $3.20 \mathrm{E}-02$ & AL-05 & Copper \\
\hline $2.80 \mathrm{~F}+04$ & AL-05 & Cobalt \\
\hline
\end{tabular}

$\begin{array}{llll}\text { GD } & 1.77 \mathrm{E}+02 & \text { GD-03 } & \text { Nickel } \\ \text { GD } & 3.01 \mathrm{E}+02 & \text { GD-03 } & \text { Np-237 } \\ \text { GD } & 3.74 \mathrm{E}+02 & & \\ \text { GD } & 2.15 \mathrm{E}+00 & & \\ \text { GD } & 2.23 \mathrm{E}+03 & \text { GD-03 } & \text { Cobalt }\end{array}$

$\begin{array}{lll}2.74 \mathrm{E}+02 & \text { AL-06 } & \text { Nickel } \\ 4.21 \mathrm{E}+00 & \text { AL-06 } & \text { Iron } \\ 8.72 \mathrm{E}+02 & & \\ 8.24 \mathrm{E}-02 & \text { AL-06 } & \text { Copper } \\ 2.13 \mathrm{E}+05 & \text { AL-06 } & \text { Cobalt }\end{array}$

$\begin{array}{lll}3.50 \mathrm{E}+02 & \text { AL-07 } & \text { Nickel } \\ 6.03 \mathrm{E}+00 & \text { AL-07 } & \text { Iron } \\ 1.64 \mathrm{E}+02 & & \\ 1.51 \mathrm{E}-01 & \text { AL-07 } & \text { Copper } \\ 3.98 \mathrm{E}+04 & \text { AL-07 } & \text { Cobalt }\end{array}$

$\begin{array}{llll}\text { GD } & 4.51 \mathrm{E}+02 & \text { GD-04 } & \text { Nickel } \\ \text { GD } & 3.33 \mathrm{E}+02 & \text { GD-04 } & \text { Np-237 } \\ \text { GD } & 4.37 \mathrm{E}+02 & & \\ \text { GD } & 2.14 \mathrm{E}+00 & & \\ \text { GD } & 3.21 \mathrm{E}+03 & \text { GD-04 } & \text { Cobalt }\end{array}$


Table A.1. (continued)

\begin{tabular}{|c|c|c|c|c|c|}
\hline $\begin{array}{l}\text { Dosimeter } \\
\text { capsule }\end{array}$ & Reaction & Cover & $\begin{array}{l}\text { Specific } \\
\text { activity } \\
\text { (Bq/mg) }\end{array}$ & Vial ID & $\begin{array}{c}\text { Dosimeter } \\
\text { material }\end{array}$ \\
\hline \multicolumn{6}{|l|}{ DOS4-3 } \\
\hline & NI58 (N,P) CO58 & & $6.71 \mathrm{E}+02$ & AL-08 & Nickel \\
\hline & FE54 (N,P) MN54 & & $1.08 \mathrm{E}+01$ & AL-08 & Iron \\
\hline & FE58 (N,G) FE59 & & $9.65 \mathrm{E}+02$ & & \\
\hline & CU63 (N,A) CO60 & & $2.50 \mathrm{E}-01$ & AL-08 & Copper \\
\hline & $\operatorname{CO} 59(\mathrm{~N}, \mathrm{G}) \mathrm{CO} 60$ & & $2.35 \mathrm{E}+05$ & AL-08 & Cobalt \\
\hline \multicolumn{6}{|l|}{ DOS5-1 } \\
\hline & NI58 (N,P) CO58 & & $2.08 \mathrm{E}+02$ & AL-09 & Nickel \\
\hline & FE54 (N,P) MN54 & & $3.38 \mathrm{E}+00$ & AL-09 & Iron \\
\hline & FE58 (N,G) FE59 & & $1.76 \mathrm{E}+02$ & & \\
\hline & CU63 (N,A) CO60 & & $6.87 \mathrm{E}-02$ & AL-09 & Copper \\
\hline & $\operatorname{CO} 59(\mathrm{~N}, \mathrm{G}) \mathrm{CO} 60$ & & $5.88 \mathrm{E}+01$ & AL-09 & $\mathrm{Co} / \mathrm{Al}$ \\
\hline & $\operatorname{CO} 59(\mathrm{~N}, \mathrm{G}) \mathrm{CO} 60$ & & $4.20 \mathrm{E}+04$ & AL-09 & Cobalt \\
\hline \multicolumn{6}{|l|}{ DOS5-2 } \\
\hline & NI58 (N,P) CO58 & GD & $3.05 \mathrm{E}+02$ & GD-05 & Nickel \\
\hline & NP237(N,F) ZR95 & GD & $2.85 \mathrm{E}+02$ & GD-05 & Np-237 \\
\hline & NP237(N,F) RU103 & GD & $3.67 \mathrm{E}+02$ & & \\
\hline & NP237(N,F) CS137 & GD & $2.03 \mathrm{E}+00$ & & \\
\hline & $\operatorname{CO} 59(\mathrm{~N}, \mathrm{G}) \mathrm{CO} 60$ & GD & $5.69 \mathrm{E}+00$ & GD-05 & $\mathrm{Co} / \mathrm{Al}$ \\
\hline & $\operatorname{CO} 59(\mathrm{~N}, \mathrm{G}) \mathrm{CO} 60$ & GD & $2.68 \mathrm{E}+03$ & GD-05 & Cobalt \\
\hline \multicolumn{6}{|l|}{ DOS5-3 } \\
\hline & NI58 (N,P) CO58 & & $4.73 \mathrm{E}+02$ & AL-10 & Nickel \\
\hline & FE54 (N,P) MN54 & & $7.63 \mathrm{E}+00$ & AL-10 & Iron \\
\hline & FE58 (N,G) FE59 & & $9.30 \mathrm{E}+02$ & & \\
\hline & CU63 (N,A) CO60 & & $1.51 \mathrm{E}-01$ & AL-10 & Copper \\
\hline & $\operatorname{CO} 59(\mathrm{~N}, \mathrm{G}) \mathrm{CO} 60$ & & $2.84 \mathrm{E}+02$ & AL-10 & $\mathrm{Co} / \mathrm{Al}$ \\
\hline & $\operatorname{CO} 59(\mathrm{~N}, \mathrm{G}) \mathrm{CO} 60$ & & $2.23 \mathrm{E}+05$ & AL-10 & Cobalt \\
\hline \multicolumn{6}{|l|}{ DOS6-1 } \\
\hline & NI58 (N,P) CO58 & & $3.13 \mathrm{E}+01$ & AL-11 & Nickel \\
\hline & FE54 (N,P) MN54 & & $5.56 \mathrm{E}-01$ & AL-11 & Iron \\
\hline & FE58 (N,G) FE59 & & $1.02 \mathrm{E}+00$ & & \\
\hline & CU63 (N,A) CO60 & & $2.20 \mathrm{E}-02$ & AL-11 & Copper \\
\hline & $\operatorname{CO} 59(\mathrm{~N}, \mathrm{G}) \operatorname{CO} 60$ & & $3.61 \mathrm{E}-01$ & AL-11 & $\mathrm{Co} / \mathrm{Al}$ \\
\hline & $\operatorname{CO} 59(\mathrm{~N}, \mathrm{G}) \mathrm{CO} 60$ & & $2.74 \mathrm{E}+02$ & AL-11 & Cobalt \\
\hline
\end{tabular}


Table A.1. (continued)

\begin{tabular}{|c|c|c|c|c|c|}
\hline $\begin{array}{l}\text { Dosimeter } \\
\text { capsule }\end{array}$ & Reaction & Cover & $\begin{array}{l}\text { Specific } \\
\text { activity } \\
\text { (Bq/mg) }\end{array}$ & Vial ID & $\begin{array}{c}\text { Dosimeter } \\
\text { material }\end{array}$ \\
\hline \multicolumn{6}{|l|}{ DOS6-2 } \\
\hline & NI58 (N,P) CO58 & GD & $3.16 \mathrm{E}+01$ & GD-06 & Nickel \\
\hline & NP237(N,F) ZR95 & GD & $1.30 \mathrm{E}+02$ & GD-06 & $\mathrm{Np}-237$ \\
\hline & NP237(N,F) RU103 & GD & $1.48 \mathrm{E}+02$ & & \\
\hline & NP237(N,F) CS137 & GD & $7.88 \mathrm{E}-01$ & & \\
\hline & $\operatorname{CO} 59(\mathrm{~N}, \mathrm{G}) \mathrm{CO} 60$ & GD & 2.97E-02 & GD-06 & $\mathrm{Co} / \mathrm{Al}$ \\
\hline & $\mathrm{CO} 59(\mathrm{~N}, \mathrm{G}) \mathrm{CO} 60$ & GD & $1.30 \mathrm{E}+01$ & GD-06 & Cobalt \\
\hline \multicolumn{6}{|l|}{ DOS6-3 } \\
\hline & NI58 (N,P) CO58 & & $3.11 \mathrm{E}+01$ & AL-12 & Nickel \\
\hline & FE54 (N,P) MN54 & & $5.42 \mathrm{E}-01$ & AL-12 & Iron \\
\hline & FE58 (N,G) FE59 & & $8.88 \mathrm{E}-01$ & & \\
\hline & CU63 (N,A) CO60 & & $2.22 \mathrm{E}-02$ & AL-12 & Copper \\
\hline & $\operatorname{CO} 59(\mathrm{~N}, \mathrm{G}) \mathrm{CO} 60$ & & $3.14 \mathrm{E}-01$ & Al-12 & $\mathrm{Co} / \mathrm{Al}$ \\
\hline & $\operatorname{CO} 59(\mathrm{~N}, \mathrm{G}) \mathrm{CO} 60$ & & $2.42 \mathrm{E}+02$ & AL-12 & Cobalt \\
\hline \multicolumn{6}{|l|}{ DOS8 } \\
\hline & NI58 (N,P) CO58 & & $1.44 \mathrm{E}+04$ & AL-15 & Nickel \\
\hline & FE54 (N,P) MN54 & & $2.39 \mathrm{E}+02$ & AL-15 & Iron \\
\hline & FE58 (N,G) FE59 & & $2.39 \mathrm{E}+04$ & & \\
\hline & CU63 (N,A) CO60 & & $4.03 \mathrm{E}+00$ & AL-15 & Copper \\
\hline & $\mathrm{CO} 59(\mathrm{~N}, \mathrm{G}) \mathrm{CO} 60$ & & $5.10 \mathrm{E}+06$ & AL-15 & Cobalt \\
\hline & NI58 (N,P) CO58 & GD & $1.45 \mathrm{E}+04$ & GD-08 & Nickel \\
\hline & NP237(N,F) ZR95 & GD & $7.51 \mathrm{E}+03$ & GD-08 & Np-237 \\
\hline & NP237(N,F) RU103 & GD & $1.06 \mathrm{E}+04$ & & \\
\hline & NP237(N,F) CS137 & GD & $5.80 \mathrm{E}+01$ & & \\
\hline & NP237(N,F) CE141 & GD & $9.07 \mathrm{E}+03$ & & \\
\hline & $\operatorname{CO} 59(\mathrm{~N}, \mathrm{G}) \mathrm{CO} 60$ & GD & $1.04 \mathrm{E}+05$ & GD-08 & Cobalt \\
\hline & NI58 (N,P) CO58 & & $1.44 \mathrm{E}+04$ & AL-16 & Nickel \\
\hline & FE54 (N,P) MN54 & & $2.44 \mathrm{E}+02$ & AL-16 & Iron \\
\hline & FE58 (N,G) FE59 & & $2.45 \mathrm{E}+04$ & & \\
\hline & CU63 (N,A) CO60 & & $4.24 \mathrm{E}+00$ & AL-16 & Copper \\
\hline & $\mathrm{CO} 59(\mathrm{~N}, \mathrm{G}) \mathrm{CO} 60$ & & $5.14 \mathrm{E}+06$ & AL-16 & Cobalt \\
\hline
\end{tabular}


Table A.1. (continued)

\begin{tabular}{|c|c|c|c|c|c|}
\hline $\begin{array}{l}\text { Dosimeter } \\
\text { capsule }\end{array}$ & Reaction & Cover & $\begin{array}{l}\text { Specific } \\
\text { activity } \\
\text { (Bq/mg) }\end{array}$ & Vial ID & $\begin{array}{c}\text { Dosimeter } \\
\text { material }\end{array}$ \\
\hline \multicolumn{6}{|l|}{ DOS9 } \\
\hline & NI58 (N,P) CO58 & & $1.38 \mathrm{E}+04$ & AL-17 & Nickel \\
\hline & FE54 (N,P) MN54 & & $2.30 \mathrm{E}+02$ & AL-17 & Iron \\
\hline & FE58 $(\mathrm{N}, \mathrm{G})$ FE59 & & $2.50 \mathrm{E}+04$ & & \\
\hline & CU63 (N,A) CO60 & & $4.03 \mathrm{E}+00$ & AL-17 & Copper \\
\hline & $\operatorname{CO} 59(\mathrm{~N}, \mathrm{G}) \mathrm{CO} 60$ & & $7.49 \mathrm{E}+03$ & AL-17 & $\mathrm{Co} / \mathrm{Al}$ \\
\hline & $\operatorname{CO} 59(\mathrm{~N}, \mathrm{G}) \mathrm{CO} 60$ & & $5.34 \mathrm{E}+06$ & AL-17 & Cobalt \\
\hline & NI58 (N,P) CO58 & GD & $1.46 \mathrm{E}+04$ & GD-09 & Nickel \\
\hline & NP237(N,F) ZR95 & GD & $7.66 \mathrm{E}+03$ & GD-09 & $\mathrm{Np}-237$ \\
\hline & NP237(N,F) RU103 & GD & $1.08 \mathrm{E}+04$ & & \\
\hline & NP237(N,F) CS137 & GD & $5.90 \mathrm{E}+01$ & & \\
\hline & NP237(N,F) CE141 & GD & $8.89 \mathrm{E}+03$ & & \\
\hline & $\operatorname{CO} 59(\mathrm{~N}, \mathrm{G}) \mathrm{CO} 60$ & GD & $2.24 \mathrm{E}+02$ & GD-09 & $\mathrm{Co} / \mathrm{Al}$ \\
\hline & $\operatorname{CO} 59(\mathrm{~N}, \mathrm{G}) \mathrm{CO} 60$ & GD & $1.06 \mathrm{E}+05$ & GD-09 & Cobalt \\
\hline & NI58 (N,P) CO58 & & $1.46 \mathrm{E}+04$ & AL-18 & Nickel \\
\hline & FE54 (N,P) MN54 & & $2.41 \mathrm{E}+02$ & AL-18 & Iron \\
\hline & FE58 (N,G) FE59 & & $2.65 \mathrm{E}+04$ & & \\
\hline & CU63 (N,A) CO60 & & $4.02 \mathrm{E}+00$ & AL-18 & Copper \\
\hline & $\operatorname{CO} 59(\mathrm{~N}, \mathrm{G}) \mathrm{CO} 60$ & & $8.03 \mathrm{E}+03$ & AL-18 & $\mathrm{Co} / \mathrm{Al}$ \\
\hline & $\operatorname{CO} 59(\mathrm{~N}, \mathrm{G}) \mathrm{CO} 60$ & & $5.61 \mathrm{E}+06$ & AL-18 & Cobalt \\
\hline \multicolumn{6}{|c|}{ HRB2-1GW } \\
\hline & NI58 (N,P) CO58 & GD & $1.63 \mathrm{E}+02$ & GD-15 & Nickel \\
\hline & NP237(N,F) ZR95 & GD & $2.12 \mathrm{E}+02$ & GD-15 & Np-237 \\
\hline & NP237(N,F) RU103 & GD & $2.58 \mathrm{E}+02$ & & \\
\hline & NP237(N,F) CS137 & GD & $1.77 \mathrm{E}+00$ & & \\
\hline & $\operatorname{CO} 59(\mathrm{~N}, \mathrm{G}) \mathrm{CO} 60$ & GD & $1.31 \mathrm{E}+03$ & GD-15 & Cobalt \\
\hline \multicolumn{6}{|c|}{ HRB2-1GC } \\
\hline & NI58 (N,P) CO58 & GD & $4.57 \mathrm{E}+03$ & GD-14 & Nickel \\
\hline & NP237(N,F) ZR95 & GD & $2.27 \mathrm{E}+03$ & GD-14 & $\mathrm{Np}-237$ \\
\hline & NP237(N,F) RU103 & GD & $4.56 \mathrm{E}+03$ & & \\
\hline & NP237(N,F) CS137 & GD & $1.58 \mathrm{E}+01$ & & \\
\hline & $\operatorname{CO} 59(\mathrm{~N}, \mathrm{G}) \mathrm{CO} 60$ & GD & $6.14 \mathrm{E}+01$ & GD-14 & $\mathrm{Co} / \mathrm{Al}$ \\
\hline & $\operatorname{CO} 59(\mathrm{~N}, \mathrm{G}) \mathrm{CO} 60$ & GD & $2.58 \mathrm{E}+04$ & GD-14 & Cobalt \\
\hline \multicolumn{6}{|c|}{ HRB2-2GW } \\
\hline & NI58 (N,P) CO58 & GD & $1.82 \mathrm{E}+02$ & GD-17 & Nickel \\
\hline & NP237(N,F) ZR95 & GD & $2.27 \mathrm{E}+02$ & GD-17 & Np-237 \\
\hline & NP237(N,F) RU103 & GD & $2.93 \mathrm{E}+02$ & & \\
\hline & NP237(N,F) CS137 & GD & $1.57 \mathrm{E}+00$ & & \\
\hline & $\operatorname{CO} 59(\mathrm{~N}, \mathrm{G}) \mathrm{CO} 60$ & GD & $1.27 \mathrm{E}+03$ & GD-17 & Cobalt \\
\hline
\end{tabular}


Table A.1. (continued)

\begin{tabular}{|c|c|c|c|c|c|}
\hline $\begin{array}{l}\text { Dosimeter } \\
\text { capsule }\end{array}$ & Reaction & Cover & $\begin{array}{l}\text { Specific } \\
\text { activity } \\
\text { (Bq/mg) }\end{array}$ & Vial ID & $\begin{array}{c}\text { Dosimeter } \\
\text { material }\end{array}$ \\
\hline \multicolumn{6}{|l|}{ HRB2-2GC } \\
\hline & NI58 (N,P) CO58 & GD & $5.29 \mathrm{E}+03$ & GD-16 & Nickel \\
\hline & NP237(N,F) ZR95 & GD & $2.64 \mathrm{E}+03$ & GD-16 & $\mathrm{Np}-237$ \\
\hline & NP237(N,F) RU103 & GD & $2.63 \mathrm{E}+03$ & & (Previous meas.) \\
\hline & NP237(N,F) CS137 & GD & $2.10 \mathrm{E}+01$ & & \\
\hline & NP237(N,F) CE141 & GD & $2.55 \mathrm{E}+03$ & & \\
\hline & $\mathrm{CO} 59(\mathrm{~N}, \mathrm{G}) \mathrm{CO} 60$ & GD & $3.56 \mathrm{E}+04$ & GD-16 & Cobalt \\
\hline \multicolumn{6}{|c|}{ HRB2-3GW } \\
\hline & NI58 (N,P) CO58 & GD & $2.09 \mathrm{E}+02$ & GD-18 & Nickel \\
\hline & NP237(N,F) ZR95 & GD & $2.58 \mathrm{E}+02$ & GD-18 & Np-237 \\
\hline & NP237(N,F) RU103 & GD & $3.22 \mathrm{E}+02$ & & \\
\hline & NP237(N,F) CS137 & GD & $1.82 \mathrm{E}+00$ & & \\
\hline & $\mathrm{CO} 59(\mathrm{~N}, \mathrm{G}) \mathrm{CO} 60$ & GD & $1.08 \mathrm{E}+03$ & GD-18 & Cobalt \\
\hline
\end{tabular}

${ }^{a}$ The dosimeter capsule IDs are followed by the number of the vial position in the capsule, where necessary. For example: DOS1-1 indicates position 1 in DOS capsule 1. Positions are indicated only for the capsules where it is necessary to analyze the positions inside the single capsule separately due to the neutron and gamma-ray flux gradients across the capsule. For the tubular capsules (e.g., HRB2-1GW), the letter appended to the end of the capsule ID indicates the axial location corresponding to the corner $(\mathrm{C})$ or weld $(\mathrm{W})$ of the beamline nozzle.

${ }^{b} \mathrm{Co} / \mathrm{Al}$ indicates aluminum-cobalt alloy with $0.100 \mathrm{wt} \% \mathrm{Co}$. 
Table A.2. Measured specific activities of the dosimeters irradiated during HFIR fuel cycle 401

\begin{tabular}{|c|c|c|c|c|}
\hline $\begin{array}{l}\text { Dosimeter } \\
\text { capsule }\end{array}$ & Reaction & $\begin{array}{l}\text { Specific } \\
\text { activity } \\
\text { (Bq/mg) }\end{array}$ & Vial ID & $\begin{array}{c}\text { Dosimeter } \\
\text { material }\end{array}$ \\
\hline \multicolumn{5}{|l|}{ HRB2-1AC } \\
\hline & NI58 (N,P ) CO58 & $4.32 \mathrm{E}+03$ & AL-27 & Nickel \\
\hline & FE54 (N,P ) MN54 & $7.19 \mathrm{E}+01$ & AL-27 & Iron \\
\hline & FE58 (N,G ) FE59 & $1.27 \mathrm{E}+04$ & & \\
\hline & CU63 (N,A ) CO60 & $1.36 \mathrm{E}+00$ & AL-27 & Copper \\
\hline & $\operatorname{CO} 59(\mathrm{~N}, \mathrm{G}) \mathrm{CO} 60$ & $3.73 \mathrm{E}+03$ & AL-27 & $\mathrm{Co} / \mathrm{Al}$ \\
\hline & $\operatorname{CO} 59(\mathrm{~N}, \mathrm{G}) \mathrm{CO} 60$ & $3.46 \mathrm{E}+06$ & AL-27 & Cobalt \\
\hline \multicolumn{5}{|l|}{ HRB2-1AW } \\
\hline & NI58 (N,P ) CO58 & $1.62 \mathrm{E}+02$ & AL-28 & Nickel \\
\hline & FE54 (N,P ) MN54 & $2.82 \mathrm{E}+00$ & AL-28 & Iron \\
\hline & FE58 (N,G ) FE59 & $1.36 \mathrm{E}+02$ & & \\
\hline & CU63 (N,A ) CO60 & $5.64 \mathrm{E}-02$ & AL-28 & Copper \\
\hline & $\operatorname{CO} 59(\mathrm{~N}, \mathrm{G}) \mathrm{CO} 60$ & $3.41 \mathrm{E}+04$ & AL-28 & Cobalt \\
\hline \multicolumn{5}{|l|}{ HRB2-2AC } \\
\hline & NI58 (N,P ) CO58 & $5.27 \mathrm{E}+03$ & AL-29 & Nickel \\
\hline & FE54 (N,P ) MN54 & $8.53 \mathrm{E}+01$ & AL-29 & Iron \\
\hline & FE58 (N,G ) FE59 & $1.81 \mathrm{E}+04$ & & \\
\hline & CU63 (N,A ) CO60 & $1.70 \mathrm{E}+00$ & AL-29 & Copper \\
\hline & $\operatorname{CO} 59(\mathrm{~N}, \mathrm{G}) \mathrm{CO} 60$ & $4.03 \mathrm{E}+06$ & AL-29 & Cobalt \\
\hline \multicolumn{5}{|l|}{ HRB2-2AW } \\
\hline & NI58 (N,P ) CO58 & $1.87 \mathrm{E}+02$ & AL-30 & Nickel \\
\hline & FE54 (N,P ) MN54 & $3.14 \mathrm{E}+00$ & AL-30 & Iron \\
\hline & FE58 (N,G ) FE59 & $1.39 \mathrm{E}+02$ & & \\
\hline & CU63 (N,A ) CO60 & $6.12 \mathrm{E}-02$ & AL-30 & Copper \\
\hline & $\operatorname{CO} 59(\mathrm{~N}, \mathrm{G}) \mathrm{CO} 60$ & $3.57 \mathrm{E}+04$ & AL-30 & Cobalt \\
\hline \multicolumn{5}{|l|}{ HRB2-3AW } \\
\hline & NI58 (N,P ) CO58 & $2.07 \mathrm{E}+02$ & AL-31 & Nickel \\
\hline & FE54 (N,P ) MN54 & $3.36 \mathrm{E}+00$ & AL-31 & Iron \\
\hline & FE58 (N,G ) FE59 & $1.22 \mathrm{E}+02$ & & \\
\hline & CU63 (N,A ) CO60 & $6.75 \mathrm{E}-02$ & AL-31 & Copper \\
\hline & $\operatorname{CO} 59(\mathrm{~N}, \mathrm{G}) \mathrm{CO} 60$ & $2.89 \mathrm{E}+04$ & AL-31 & Cobalt \\
\hline
\end{tabular}


Table A.3. Measured helium concentrations in Be HAFMs

\begin{tabular}{|c|c|c|c|c|c|c|}
\hline \multirow[t]{2}{*}{ Sample } & \multirow[t]{2}{*}{$\begin{array}{c}\text { Mass }^{a} \\
\text { (mg) }\end{array}$} & \multirow{2}{*}{$\begin{array}{c}\text { Measured } \\
{ }^{4} \mathrm{He} \\
\left(10^{11} \text { atoms }\right)\end{array}$} & \multicolumn{4}{|c|}{$\begin{array}{c}\text { Measured } \\
\text { helium concentration } \\
\text { (appb) }^{b}\end{array}$} \\
\hline & & & Measured & & vera & $g^{c}$ \\
\hline DOS-1-GD-01 ${ }^{\mathrm{d}}$ & $\begin{array}{l}2.16 \\
1.19\end{array}$ & $\begin{array}{r}11.13 \\
6.081\end{array}$ & $\begin{array}{l}7.71 \\
7.65\end{array}$ & 7.68 & \pm & 0.04 \\
\hline DOS-2-GD-02 & $\begin{array}{l}2.57 \\
1.68\end{array}$ & $\begin{array}{r}11.01 \\
7.360\end{array}$ & $\begin{array}{l}6.41 \\
6.56\end{array}$ & 6.49 & \pm & 0.11 \\
\hline DOS-3-GD-03 & $\begin{array}{l}1.67 \\
1.40\end{array}$ & $\begin{array}{l}4.036 \\
3.211\end{array}$ & $\begin{array}{l}3.62 \\
3.43\end{array}$ & 3.53 & \pm & 0.13 \\
\hline DOS-4-GD-04 & $\begin{array}{l}1.75 \\
2.06\end{array}$ & $\begin{array}{l}4.351 \\
5.063\end{array}$ & $\begin{array}{l}3.72 \\
3.68\end{array}$ & 3.70 & \pm & 0.03 \\
\hline DOS-5-GD-05 & $\begin{array}{l}2.63 \\
1.88\end{array}$ & $\begin{array}{l}5.489 \\
3.856\end{array}$ & $\begin{array}{l}3.12 \\
3.07\end{array}$ & 3.10 & \pm & 0.04 \\
\hline DOS-6-GD-06 & $\begin{array}{l}2.16 \\
1.55\end{array}$ & $\begin{array}{l}3.559 \\
2.639\end{array}$ & $\begin{array}{l}2.47 \\
2.55\end{array}$ & 2.51 & \pm & 0.06 \\
\hline DOS-8-GD-08 & $\begin{array}{l}1.68 \\
1.89\end{array}$ & $\begin{array}{l}76.97 \\
85.90\end{array}$ & $\begin{array}{l}68.57 \\
68.02\end{array}$ & 68.3 & \pm & 0.4 \\
\hline DOS-9-GD-09 & $\begin{array}{l}1.83 \\
2.01\end{array}$ & $\begin{array}{l}86.23 \\
95.60\end{array}$ & $\begin{array}{l}70.52 \\
71.18\end{array}$ & 70.9 & \pm & 0.5 \\
\hline HRB2-1G-GD-14 & $\begin{array}{l}1.31 \\
1.37\end{array}$ & $\begin{array}{l}25.20 \\
26.36\end{array}$ & $\begin{array}{l}28.79 \\
28.80\end{array}$ & 28.8 & \pm & 0.1 \\
\hline HRB2-1G-GD-15 & $\begin{array}{l}1.71 \\
1.79\end{array}$ & $\begin{array}{l}2.872 \\
3.039\end{array}$ & $\begin{array}{l}2.51 \\
2.54\end{array}$ & 2.53 & \pm & 0.02 \\
\hline HRB2-2G-GD-16 & $\begin{array}{l}1.42 \\
1.59\end{array}$ & $\begin{array}{l}25.81 \\
28.74\end{array}$ & $\begin{array}{l}27.20 \\
27.05\end{array}$ & 27.1 & \pm & 0.1 \\
\hline HRB2-2G-GD-17 & $\begin{array}{l}1.70 \\
1.53\end{array}$ & $\begin{array}{l}3.138 \\
2.902\end{array}$ & $\begin{array}{l}2.76 \\
2.84\end{array}$ & 2.80 & \pm & 0.06 \\
\hline HRB2-3G-GD-18 & $\begin{array}{l}1.69 \\
1.13\end{array}$ & $\begin{array}{l}3.715 \\
2.486\end{array}$ & $\begin{array}{l}3.29 \\
3.29\end{array}$ & 3.29 & \pm & $<0.01$ \\
\hline
\end{tabular}

${ }^{a}$ Mass of specimen for analysis. Mass uncertainty is $\pm 0.02 \mathrm{mg}$.

${ }^{b}$ Helium concentration in atomic parts per billion $\left(10^{-9}\right.$ atom fraction) with respect to the total number of atoms in the specimen. Uncertainty is estimated to be $\pm 1 \%$.

${ }^{c}$ Mean and standard deviation $(1 \sigma)$ of replicate analyses.

${ }^{d}$ The ID in the form capsule-vial is used here; for example, DOS-1-GD-01 denotes vial GD-01 in the capsule DOS-1. 
Table A.4. Reactor power vs time for cycle 400 (100\% indicates $85 \mathrm{MW}$ )

\begin{tabular}{ccc}
\hline & Power history \\
\hline Date & Time & Action \\
\hline $4 / 27 / 04$ & $03: 57$ & $100 \%$ \\
$4 / 27 / 04$ & $04: 42$ & Shutdown \\
$4 / 27 / 04$ & $09: 26$ & $100 \%$ \\
$4 / 27 / 04$ & $10: 20$ & Shutdown \\
$4 / 27 / 04$ & $12: 31$ & $100 \%$ \\
$5 / 21 / 04$ & $17: 35$ & Shutdown \\
\hline \multicolumn{3}{c}{ Irradiation time at $100 \%$ reactor } \\
\multicolumn{3}{c}{ power } \\
\hline
\end{tabular}

Table A.5. Reactor power vs time for cycle 401 (100\% indicates $85 \mathrm{MW}$ )

\begin{tabular}{ccc}
\hline \multicolumn{3}{c}{ Power History } \\
\hline Date & Time & Action \\
\hline $7 / 15 / 04$ & $21: 52$ & $100 \%$ \\
$7 / 15 / 04$ & $23: 07$ & Shutdown \\
$7 / 15 / 04$ & $23: 38$ & $30 \%$ \\
$7 / 16 / 04$ & $00: 00$ & Shutdown \\
$7 / 16 / 04$ & $00: 26$ & $100 \%$ \\
$8 / 9 / 04$ & $07: 37$ & Shutdown \\
\hline Irradiation time at $100 \%$ & \\
reactor power & \\
\hline
\end{tabular}





\section{APPENDIX B}

MULTIGROUP NEUTRON AND GAMMA-RAY FLUXES OBTAINED FROM TRANSPORT CALCULATIONS 

The transport calculations were performed with 61 neutron and 23 gamma-ray energy groups. Tables B.1 and B.2 provide the energy boundaries for the neutron and gamma-ray energy groups, respectively. Details about the calculations are provided in Refs. 6, 13, and 14.

Tables B.3 to B.10 provide neutron and gamma-ray fluxes, normalized to a reactor power of $85 \mathrm{MW}$, at the locations of the dosimeters. For each location, first the 61 neutron group fluxes are given, starting with the highest energy group, and then the 23 gamma-ray group fluxes are listed, again starting with the highest energy group. Fluxes are given for each dosimetry capsule. If the capsule contained multiple dosimetry vials and the calculations indicated considerable variation in the field from vial to vial, the fluxes are given for each vial location in the capsule (e.g., see Table B.3, for capsule DOS-1). The numbering of the vial locations inside the capsules is consistent with Fig. 2.

Where the dosimetry capsules were located in symmetric (equivalent) positions, and consequently the calculations provided equal fluxes, only one set of calculated fluxes is given for all the capsules (e.g., see Table B.3, which provides calculated fluxes for the DOS-1 and DOS-2 capsules).

The calculated fluxes reported here are cycle-averaged for all Key 2 and Key 7 locations and are for the end-of-cycle for Key 3. All fluxes are normalized to the core power of $85 \mathrm{MW}$.

Table B.1. Energy boundaries for 61 neutron groups used in transport calculations $(\mathrm{eV})$

\begin{tabular}{llllll}
\hline $1.4920 \mathrm{E}+07$ & $1.2210 \mathrm{E}+07$ & $1.0000 \mathrm{E}+07$ & $8.1870 \mathrm{E}+06$ & $6.7030 \mathrm{E}+06$ & $5.4880 \mathrm{E}+06$ \\
$4.4930 \mathrm{E}+06$ & $3.6790 \mathrm{E}+06$ & $3.0120 \mathrm{E}+06$ & $2.4660 \mathrm{E}+06$ & $2.3460 \mathrm{E}+06$ & $2.2310 \mathrm{E}+06$ \\
$2.0190 \mathrm{E}+06$ & $1.6530 \mathrm{E}+06$ & $1.3530 \mathrm{E}+06$ & $1.1080 \mathrm{E}+06$ & $9.0720 \mathrm{E}+05$ & $7.4270 \mathrm{E}+05$ \\
$6.0810 \mathrm{E}+05$ & $5.2340 \mathrm{E}+05$ & $4.9790 \mathrm{E}+05$ & $3.8770 \mathrm{E}+05$ & $3.0200 \mathrm{E}+05$ & $2.9850 \mathrm{E}+05$ \\
$2.9720 \mathrm{E}+05$ & $2.9450 \mathrm{E}+05$ & $2.7320 \mathrm{E}+05$ & $2.2370 \mathrm{E}+05$ & $1.8320 \mathrm{E}+05$ & $1.5000 \mathrm{E}+05$ \\
$1.2280 \mathrm{E}+05$ & $8.6520 \mathrm{E}+04$ & $5.6560 \mathrm{E}+04$ & $5.2480 \mathrm{E}+04$ & $3.4310 \mathrm{E}+04$ & $2.8500 \mathrm{E}+04$ \\
$2.7000 \mathrm{E}+04$ & $2.6060 \mathrm{E}+04$ & $2.4790 \mathrm{E}+04$ & $2.3580 \mathrm{E}+04$ & $1.5030 \mathrm{E}+04$ & $9.1190 \mathrm{E}+03$ \\
$5.5310 \mathrm{E}+03$ & $3.7070 \mathrm{E}+03$ & $3.0350 \mathrm{E}+03$ & $2.6130 \mathrm{E}+03$ & $2.2490 \mathrm{E}+03$ & $2.0350 \mathrm{E}+03$ \\
$1.2340 \mathrm{E}+03$ & $7.4850 \mathrm{E}+02$ & $4.5400 \mathrm{E}+02$ & $2.7540 \mathrm{E}+02$ & $1.6700 \mathrm{E}+02$ & $1.0130 \mathrm{E}+02$ \\
$4.7850 \mathrm{E}+01$ & $2.2600 \mathrm{E}+01$ & $1.0680 \mathrm{E}+01$ & $5.0430 \mathrm{E}+00$ & $2.3820 \mathrm{E}+00$ & $1.1250 \mathrm{E}+00$ \\
$4.1400 \mathrm{E}-01$ & $1.00 \mathrm{E}-05$ & & & & \\
\hline
\end{tabular}

Table B.2. Energy boundaries for 23 gamma-ray groups used in transport calculations $(\mathrm{eV})$

\begin{tabular}{llllll}
\hline $1.40 \mathrm{E}+07$ & $1.00 \mathrm{E}+07$ & $8.00 \mathrm{E}+06$ & $7.50 \mathrm{E}+06$ & $7.00 \mathrm{E}+06$ & $6.00 \mathrm{E}+06$ \\
$5.00 \mathrm{E}+06$ & $4.00 \mathrm{E}+06$ & $3.00 \mathrm{E}+06$ & $2.50 \mathrm{E}+06$ & $2.00 \mathrm{E}+06$ & $1.50 \mathrm{E}+06$ \\
$1.00 \mathrm{E}+06$ & $7.00 \mathrm{E}+05$ & $6.00 \mathrm{E}+05$ & $5.10 \mathrm{E}+05$ & $4.00 \mathrm{E}+05$ & $3.00 \mathrm{E}+05$ \\
$1.50 \mathrm{E}+05$ & $1.00 \mathrm{E}+05$ & $7.00 \mathrm{E}+04$ & $4.50 \mathrm{E}+04$ & $2.00 \mathrm{E}+04$ & $1.00 \mathrm{E}+04$ \\
\hline
\end{tabular}


Table B.3. Calculated neutron and gamma fluxes normalized to the reactor power of $85 \mathrm{MW}$ for locations K2W, P8 (DOS-1) and K2W, P32 (DOS-2).

Units are $\left(\mathrm{cm}^{-2} \mathrm{~s}^{-1}\right)$.

\section{DOS1-1 and DOS2-1}

3.89E+06 1.16E+07 2.51E+07 5.10E+07 8.87E+07 1.25E+08 1.29E+08 1.55E+08 2.09E+08 $6.75 \mathrm{E}+07$ $6.61 \mathrm{E}+07 \quad 1.21 \mathrm{E}+08$ 2.79E+08 2.92E+08 3.06E+08 2.59E+08 3.00E+08 3.14E+08 2.09E+08 $6.11 \mathrm{E}+07$ $2.28 \mathrm{E}+08$ 2.72E+08 1.46E+07 5.19E+06 1.01E+07 6.23E+07 1.81E+08 1.45E+08 1.49E+08 1.35E+08 $2.01 \mathrm{E}+082.27 \mathrm{E}+08 \quad 3.41 \mathrm{E}+071.91 \mathrm{E}+08$ 4.57E+07 1.23E+07 2.80E+07 4.95E+07 2.90E+07 1.73E+08 $2.04 \mathrm{E}+08 \quad 1.80 \mathrm{E}+08 \quad 1.53 \mathrm{E}+08 \quad 8.03 \mathrm{E}+07 \quad 5.38 \mathrm{E}+07 \quad 4.67 \mathrm{E}+07 \quad 4.23 \mathrm{E}+07 \quad 2.00 \mathrm{E}+08 \quad 1.85 \mathrm{E}+08 \quad 1.91 \mathrm{E}+08$ $1.79 \mathrm{E}+08 \quad 1.96 \mathrm{E}+08 \quad 1.98 \mathrm{E}+08 \quad 3.02 \mathrm{E}+08 \quad 3.08 \mathrm{E}+08 \quad 3.13 \mathrm{E}+08 \quad 3.16 \mathrm{E}+08 \quad 3.24 \mathrm{E}+08 \quad 3.45 \mathrm{E}+08 \quad 5.00 \mathrm{E}+08$ $1.44 \mathrm{E}+10$

$1.65 \mathrm{E}+074.92 \mathrm{E}+094.72 \mathrm{E}+10 \quad 6.23 \mathrm{E}+09 \quad 5.90 \mathrm{E}+10 \quad 4.44 \mathrm{E}+10 \quad 8.62 \mathrm{E}+10 \quad 1.37 \mathrm{E}+119.44 \mathrm{E}+102.70 \mathrm{E}+11$ $2.11 \mathrm{E}+112.98 \mathrm{E}+112.76 \mathrm{E}+11 \quad 1.23 \mathrm{E}+11 \quad 2.21 \mathrm{E}+11 \quad 2.43 \mathrm{E}+11 \quad 3.19 \mathrm{E}+11 \quad 1.07 \mathrm{E}+124.28 \mathrm{E}+11 \quad 1.42 \mathrm{E}+11$ $3.31 \mathrm{E}+106.37 \mathrm{E}+08$ 4.78E+05

\section{DOS1-2 and DOS2-2}

4.09E+06 1.24E+07 2.70E+07 5.55E+07 9.78E+07 1.39E+08 1.45E+08 1.75E+08 2.36E+08 7.69E+07 $7.57 \mathrm{E}+07 \quad 1.39 \mathrm{E}+08 \quad 3.21 \mathrm{E}+08 \quad 3.37 \mathrm{E}+08 \quad 3.54 \mathrm{E}+08 \quad 3.01 \mathrm{E}+08 \quad 3.48 \mathrm{E}+08 \quad 3.65 \mathrm{E}+082.44 \mathrm{E}+087.11 \mathrm{E}+07$ $2.66 \mathrm{E}+08 \quad 3.18 \mathrm{E}+08 \quad 1.71 \mathrm{E}+07 \quad 6.07 \mathrm{E}+06 \quad 1.17 \mathrm{E}+07 \quad 7.26 \mathrm{E}+07 \quad 2.12 \mathrm{E}+08 \quad 1.69 \mathrm{E}+08 \quad 1.73 \mathrm{E}+08 \quad 1.58 \mathrm{E}+08$ $2.35 \mathrm{E}+08$ 2.67E+08 3.98E+07 2.24E+08 5.38E+07 1.45E+07 3.29E+07 5.83E+07 3.41E+07 2.03E+08 $2.39 \mathrm{E}+08 \quad 2.10 \mathrm{E}+08 \quad 1.79 \mathrm{E}+08 \quad 9.44 \mathrm{E}+07 \quad 6.32 \mathrm{E}+07 \quad 5.47 \mathrm{E}+07 \quad 4.97 \mathrm{E}+07 \quad 2.35 \mathrm{E}+082.18 \mathrm{E}+08 \quad 2.25 \mathrm{E}+08$ $2.10 \mathrm{E}+08$ 2.31E+08 2.34E+08 3.57E+08 3.63E+08 3.69E+08 3.73E+08 3.82E+08 4.06E+08 5.89E+08 $1.69 \mathrm{E}+10$ $2.69 \mathrm{E}+078.93 \mathrm{E}+099.18 \mathrm{E}+101.20 \mathrm{E}+10 \quad 1.16 \mathrm{E}+11 \quad 8.76 \mathrm{E}+10 \quad 1.70 \mathrm{E}+11 \quad 2.70 \mathrm{E}+11 \quad 1.88 \mathrm{E}+11 \quad 5.36 \mathrm{E}+11$

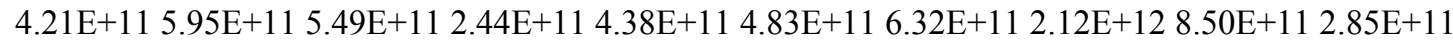
$6.74 \mathrm{E}+101.28 \mathrm{E}+098.27 \mathrm{E}+05$

\section{DOS1-3 and DOS2-3}

4.66E+06 1.44E+07 3.22E+07 6.67E+07 1.17E+08 1.66E+08 1.74E+08 2.12E+08 2.87E+08 9.36E+07 $9.25 \mathrm{E}+07 \quad 1.73 \mathrm{E}+08 \quad 4.06 \mathrm{E}+08 \quad 4.29 \mathrm{E}+08 \quad 4.53 \mathrm{E}+08 \quad 3.88 \mathrm{E}+08 \quad 4.51 \mathrm{E}+08 \quad 4.75 \mathrm{E}+08 \quad 3.16 \mathrm{E}+089.25 \mathrm{E}+07$ $3.46 \mathrm{E}+08$ 4.14E+08 2.22E+07 7.92E+06 1.53E+07 9.47E+07 2.76E+08 2.21E+08 2.27E+08 2.07E+08 $3.08 \mathrm{E}+08$ 3.50E+08 5.24E+07 2.94E+08 7.02E+07 1.88E+07 4.31E+07 7.69E+07 4.51E+07 2.67E+08 $3.16 \mathrm{E}+08 \quad 2.77 \mathrm{E}+08 \quad 2.37 \mathrm{E}+08 \quad 1.24 \mathrm{E}+08 \quad 8.34 \mathrm{E}+07 \quad 7.22 \mathrm{E}+07 \quad 6.56 \mathrm{E}+07 \quad 3.11 \mathrm{E}+082.88 \mathrm{E}+082.98 \mathrm{E}+08$ $2.78 \mathrm{E}+08$ 3.07E+08 3.10E+08 4.74E+08 4.84E+08 4.92E+08 4.99E+08 5.12E+08 5.46E+08 7.94E+08 $2.26 \mathrm{E}+10$ $2.70 \mathrm{E}+071.08 \mathrm{E}+109.74 \mathrm{E}+101.29 \mathrm{E}+101.20 \mathrm{E}+119.02 \mathrm{E}+10 \quad 1.75 \mathrm{E}+112.76 \mathrm{E}+11 \quad 1.90 \mathrm{E}+115.44 \mathrm{E}+11$

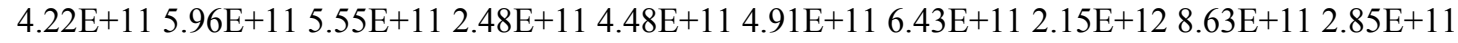
$6.50 \mathrm{E}+101.26 \mathrm{E}+091.07 \mathrm{E}+06$ 
Table B.4. Calculated neutron and gamma fluxes normalized to the reactor power of $85 \mathrm{MW}$ for location K3, P3 (DOS-3).

Units are $\left(\mathrm{cm}^{-2} \mathrm{~s}^{-1}\right)$.

\section{DOS3-1}

8.59E+05 2.36E+06 5.71E+06 9.40E+06 2.35E+07 3.94E+07 3.84E+07 5.58E+07 8.27E+07 2.95E+07 $3.04 \mathrm{E}+07 \quad 5.68 \mathrm{E}+07 \quad 1.48 \mathrm{E}+08 \quad 1.70 \mathrm{E}+08 \quad 1.88 \mathrm{E}+08 \quad 1.74 \mathrm{E}+08 \quad 2.07 \mathrm{E}+082.28 \mathrm{E}+08 \quad 1.51 \mathrm{E}+08 \quad 4.60 \mathrm{E}+07$ $1.78 \mathrm{E}+08 \quad 2.17 \mathrm{E}+08 \quad 1.13 \mathrm{E}+074.00 \mathrm{E}+06 \quad 7.76 \mathrm{E}+06 \quad 5.09 \mathrm{E}+071.47 \mathrm{E}+08 \quad 1.24 \mathrm{E}+081.25 \mathrm{E}+08 \quad 1.18 \mathrm{E}+08$ $1.78 \mathrm{E}+08 \quad 2.05 \mathrm{E}+08 \quad 3.17 \mathrm{E}+071.78 \mathrm{E}+08 \quad 4.46 \mathrm{E}+071.18 \mathrm{E}+072.80 \mathrm{E}+074.56 \mathrm{E}+072.49 \mathrm{E}+071.68 \mathrm{E}+08$ $1.94 \mathrm{E}+08 \quad 1.77 \mathrm{E}+08 \quad 1.51 \mathrm{E}+08 \quad 7.88 \mathrm{E}+07 \quad 5.38 \mathrm{E}+07 \quad 4.79 \mathrm{E}+07 \quad 4.32 \mathrm{E}+07 \quad 2.03 \mathrm{E}+08 \quad 1.97 \mathrm{E}+08 \quad 2.07 \mathrm{E}+08$ $1.95 \mathrm{E}+08 \quad 2.19 \mathrm{E}+08 \quad 2.24 \mathrm{E}+08 \quad 3.55 \mathrm{E}+08 \quad 3.76 \mathrm{E}+08 \quad 3.97 \mathrm{E}+08$ 4.19E+08 4.44E+08 4.94E+08 7.45E+08 $1.66 \mathrm{E}+10$

$1.92 \mathrm{E}+079.31 \mathrm{E}+095.19 \mathrm{E}+10 \quad 8.32 \mathrm{E}+09 \quad 5.73 \mathrm{E}+10 \quad 5.35 \mathrm{E}+10 \quad 4.88 \mathrm{E}+101.38 \mathrm{E}+117.64 \mathrm{E}+101.62 \mathrm{E}+11$ $1.88 \mathrm{E}+112.28 \mathrm{E}+112.30 \mathrm{E}+11 \quad 1.02 \mathrm{E}+11 \quad 1.93 \mathrm{E}+11 \quad 2.08 \mathrm{E}+11 \quad 2.70 \mathrm{E}+119.37 \mathrm{E}+113.72 \mathrm{E}+11 \quad 1.12 \mathrm{E}+11$ $2.36 \mathrm{E}+104.44 \mathrm{E}+086.35 \mathrm{E}+05$

\section{DOS3-2}

9.34E+05 2.53E+06 6.81E+06 1.11E+07 2.95E+07 5.08E+07 5.05E+07 7.43E+07 1.09E+08 3.94E+07 4.07E+07 7.63E+07 2.04E+08 2.36E+08 2.57E+08 $2.37 \mathrm{E}+08 \quad 2.84 \mathrm{E}+08 \quad 3.03 \mathrm{E}+08 \quad 2.01 \mathrm{E}+08 \quad 6.23 \mathrm{E}+07$ $2.43 \mathrm{E}+082.89 \mathrm{E}+08 \quad 1.45 \mathrm{E}+07 \quad 5.17 \mathrm{E}+061.02 \mathrm{E}+07 \quad 7.01 \mathrm{E}+071.98 \mathrm{E}+08 \quad 1.72 \mathrm{E}+081.69 \mathrm{E}+08 \quad 1.63 \mathrm{E}+08$ $2.44 \mathrm{E}+082.81 \mathrm{E}+08 \quad 4.45 \mathrm{E}+072.46 \mathrm{E}+08$ 7.59E+07 2.15E+07 3.77E+07 5.47E+07 3.19E+07 2.37E+08 $2.71 \mathrm{E}+08 \quad 2.62 \mathrm{E}+08 \quad 2.17 \mathrm{E}+08 \quad 1.11 \mathrm{E}+08$ 7.97E+07 7.54E+07 6.13E+07 2.90E+08 2.91E+08 3.03E+08 $3.08 \mathrm{E}+08 \quad 3.24 \mathrm{E}+08 \quad 3.40 \mathrm{E}+08$ 5.39E +08 5.78E+08 6.17E+08 6.57E+08 7.11E+08 7.97E+08 1.22E+09 $3.07 \mathrm{E}+10$ $1.77 \mathrm{E}+071.15 \mathrm{E}+105.31 \mathrm{E}+109.23 \mathrm{E}+095.40 \mathrm{E}+10 \quad 5.22 \mathrm{E}+104.77 \mathrm{E}+101.32 \mathrm{E}+117.30 \mathrm{E}+101.53 \mathrm{E}+11$ $1.78 \mathrm{E}+112.16 \mathrm{E}+112.18 \mathrm{E}+119.55 \mathrm{E}+101.85 \mathrm{E}+11 \quad 1.97 \mathrm{E}+112.55 \mathrm{E}+11 \quad 8.66 \mathrm{E}+113.37 \mathrm{E}+119.97 \mathrm{E}+10$ $1.90 \mathrm{E}+103.09 \mathrm{E}+081.05 \mathrm{E}+06$

\section{DOS3-3}

$1.16 \mathrm{E}+06$ 3.03E+06 8.94E+06 1.51E+07 4.22E+07 7.56E+07 7.65E+07 1.15E+08 1.65E+08 5.95E+07 $6.18 \mathrm{E}+071.17 \mathrm{E}+08 \quad 3.18 \mathrm{E}+08 \quad 3.72 \mathrm{E}+08 \quad 4.03 \mathrm{E}+08 \quad 3.88 \mathrm{E}+08 \quad 4.52 \mathrm{E}+08 \quad 4.66 \mathrm{E}+083.09 \mathrm{E}+08 \quad 9.62 \mathrm{E}+07$ $3.88 \mathrm{E}+08 \quad 4.59 \mathrm{E}+08 \quad 2.31 \mathrm{E}+07 \quad 8.26 \mathrm{E}+06 \quad 1.63 \mathrm{E}+07 \quad 1.10 \mathrm{E}+08 \quad 3.17 \mathrm{E}+08 \quad 2.71 \mathrm{E}+08 \quad 2.64 \mathrm{E}+08 \quad 2.61 \mathrm{E}+08$ $3.74 \mathrm{E}+08$ 4.58E+08 6.97E+07 3.82E+08 9.99E+07 2.75E+07 5.95E+07 1.07E+08 6.10E+07 3.77E+08 $4.53 \mathrm{E}+08 \quad 4.02 \mathrm{E}+08 \quad 3.52 \mathrm{E}+08 \quad 1.90 \mathrm{E}+08 \quad 1.30 \mathrm{E}+08 \quad 1.14 \mathrm{E}+08 \quad 1.02 \mathrm{E}+08 \quad 5.08 \mathrm{E}+084.92 \mathrm{E}+08 \quad 5.31 \mathrm{E}+08$ 4.95E+08 5.82E+08 6.12E+08 9.80E+08 1.05E+09 1.13E+09 1.20E+09 1.29E+09 1.44E+09 2.22E+09 $7.10 \mathrm{E}+10$ $1.81 \mathrm{E}+071.72 \mathrm{E}+10 \quad 6.39 \mathrm{E}+101.21 \mathrm{E}+105.91 \mathrm{E}+10 \quad 5.80 \mathrm{E}+10 \quad 5.43 \mathrm{E}+101.44 \mathrm{E}+117.86 \mathrm{E}+101.72 \mathrm{E}+11$ $1.91 \mathrm{E}+112.31 \mathrm{E}+112.38 \mathrm{E}+11 \quad 1.05 \mathrm{E}+11 \quad 2.03 \mathrm{E}+112.17 \mathrm{E}+112.84 \mathrm{E}+119.90 \mathrm{E}+114.18 \mathrm{E}+11 \quad 1.41 \mathrm{E}+11$ $3.21 \mathrm{E}+106.34 \mathrm{E}+082.41 \mathrm{E}+06$ 
Table B.5. Calculated neutron and gamma fluxes normalized to the reactor power of $85 \mathrm{MW}$ for location K3, P8 (DOS-4).

Units are $\left(\mathrm{cm}^{-2} \mathrm{~s}^{-1}\right)$.

\begin{abstract}
DOS4-1
2.68E+06 6.67E+06 2.50E+07 3.03E+07 7.57E+07 1.08E+08 8.87E+07 1.18E+08 1.59E+08 5.64E+07 $5.75 \mathrm{E}+07 \quad 1.03 \mathrm{E}+08 \quad 2.57 \mathrm{E}+08 \quad 2.90 \mathrm{E}+083.13 \mathrm{E}+08 \quad 2.80 \mathrm{E}+08 \quad 3.37 \mathrm{E}+08 \quad 3.63 \mathrm{E}+082.45 \mathrm{E}+08$ 7.42E+07 $2.91 \mathrm{E}+083.43 \mathrm{E}+08$ 1.72E+07 6.21E+06 1.21E+07 $8.35 \mathrm{E}+07$ 2.32E+08 2.04E+08 2.02E+08 1.90E+08 $2.91 \mathrm{E}+083.31 \mathrm{E}+08$ 5.22E $+072.92 \mathrm{E}+08 \quad 8.64 \mathrm{E}+07 \quad 2.43 \mathrm{E}+074.38 \mathrm{E}+076.23 \mathrm{E}+073.72 \mathrm{E}+072.78 \mathrm{E}+08$ $3.21 \mathrm{E}+08 \quad 3.04 \mathrm{E}+08 \quad 2.52 \mathrm{E}+08 \quad 1.30 \mathrm{E}+089.10 \mathrm{E}+07 \quad 8.43 \mathrm{E}+077.17 \mathrm{E}+073.33 \mathrm{E}+083.26 \mathrm{E}+08 \quad 3.41 \mathrm{E}+08$ $3.37 \mathrm{E}+08 \quad 3.60 \mathrm{E}+08 \quad 3.71 \mathrm{E}+08 \quad 5.82 \mathrm{E}+08 \quad 6.15 \mathrm{E}+08 \quad 6.51 \mathrm{E}+08 \quad 6.81 \mathrm{E}+08 \quad 7.24 \mathrm{E}+08 \quad 8.13 \mathrm{E}+08 \quad 1.22 \mathrm{E}+09$ $3.15 \mathrm{E}+10$

$1.77 \mathrm{E}+071.42 \mathrm{E}+10 \quad 6.45 \mathrm{E}+101.08 \mathrm{E}+10 \quad 6.15 \mathrm{E}+10 \quad 5.81 \mathrm{E}+10 \quad 5.74 \mathrm{E}+101.47 \mathrm{E}+11 \quad 8.10 \mathrm{E}+101.93 \mathrm{E}+11$ $2.00 \mathrm{E}+112.62 \mathrm{E}+112.64 \mathrm{E}+11 \quad 1.18 \mathrm{E}+112.30 \mathrm{E}+11 \quad 2.47 \mathrm{E}+11 \quad 3.25 \mathrm{E}+11 \quad 1.08 \mathrm{E}+12 \quad 4.36 \mathrm{E}+11 \quad 1.36 \mathrm{E}+11$

$2.55 \mathrm{E}+104.62 \mathrm{E}+081.14 \mathrm{E}+06$
\end{abstract}

\title{
DOS4-2
}

$2.51 \mathrm{E}+06$ 6.40E+06 2.26E+07 2.96E+07 7.55E+07 1.11E+08 9.98E+07 1.37E+08 1.91E+08 6.99E+07 7.02E+07 1.30E+08 3.39E+08 3.88E+08 4.33E+08 3.96E+08 4.68E+08 5.10E+08 3.37E+08 1.04E+08 4.07E+08 4.91E+08 2.53E+07 9.05E+06 1.74E+07 1.14E+08 3.31E+08 2.84E+08 2.83E+08 2.71E+08 $4.00 \mathrm{E}+084.68 \mathrm{E}+08$ 7.24E+07 4.00E+08 1.06E+08 $2.90 \mathrm{E}+076.28 \mathrm{E}+071.01 \mathrm{E}+085.79 \mathrm{E}+07 \quad 3.83 \mathrm{E}+08$ $4.51 \mathrm{E}+08$ 4.14E+08 3.52E+08 1.85E+08 1.28E+08 $1.15 \mathrm{E}+08$ 1.01E+08 4.83E+08 4.72E+08 4.99E+08 4.76E +08 5.33E+08 5.56E+08 8.82E+08 9.49E+08 1.01E+09 1.07E+09 1.13E+09 1.28E+09 1.92E+09 $4.26 \mathrm{E}+10$

$1.27 \mathrm{E}+071.68 \mathrm{E}+10 \quad 5.67 \mathrm{E}+101.13 \mathrm{E}+104.92 \mathrm{E}+104.88 \mathrm{E}+104.80 \mathrm{E}+101.16 \mathrm{E}+116.61 \mathrm{E}+101.53 \mathrm{E}+11$ $1.63 \mathrm{E}+112.14 \mathrm{E}+112.13 \mathrm{E}+119.82 \mathrm{E}+102.02 \mathrm{E}+112.09 \mathrm{E}+112.71 \mathrm{E}+11 \quad 8.55 \mathrm{E}+112.92 \mathrm{E}+11 \quad 6.80 \mathrm{E}+10$ $8.89 \mathrm{E}+091.50 \mathrm{E}+081.39 \mathrm{E}+06$

\section{DOS4-3}

$2.91 \mathrm{E}+06$ 7.43E+06 2.76E+07 3.57E+07 9.85E+07 1.50E+08 1.34E+08 1.92E+08 2.60E+08 9.57E+07 $9.83 \mathrm{E}+07 \quad 1.80 \mathrm{E}+08 \quad 4.86 \mathrm{E}+08 \quad 5.50 \mathrm{E}+08 \quad 5.90 \mathrm{E}+08 \quad 5.55 \mathrm{E}+08 \quad 6.50 \mathrm{E}+08 \quad 6.90 \mathrm{E}+084.54 \mathrm{E}+08 \quad 1.41 \mathrm{E}+08$ $5.68 \mathrm{E}+08 \quad 6.63 \mathrm{E}+08 \quad 3.27 \mathrm{E}+071.17 \mathrm{E}+072.29 \mathrm{E}+07 \quad 1.61 \mathrm{E}+08 \quad 4.54 \mathrm{E}+083.99 \mathrm{E}+083.89 \mathrm{E}+08 \quad 3.86 \mathrm{E}+08$ $5.60 \mathrm{E}+08 \quad 6.70 \mathrm{E}+08 \quad 1.03 \mathrm{E}+08 \quad 5.72 \mathrm{E}+081.69 \mathrm{E}+08 \quad 4.70 \mathrm{E}+07 \quad 8.91 \mathrm{E}+071.32 \mathrm{E}+08$ 7.76E $+075.58 \mathrm{E}+08$ $6.58 \mathrm{E}+08 \quad 6.23 \mathrm{E}+08 \quad 5.37 \mathrm{E}+08 \quad 2.84 \mathrm{E}+08 \quad 1.98 \mathrm{E}+08 \quad 1.79 \mathrm{E}+08 \quad 1.55 \mathrm{E}+08 \quad 7.47 \mathrm{E}+087.36 \mathrm{E}+08 \quad 7.81 \mathrm{E}+08$ $7.69 \mathrm{E}+08 \quad 8.65 \mathrm{E}+08 \quad 9.03 \mathrm{E}+08 \quad 1.45 \mathrm{E}+09 \quad 1.55 \mathrm{E}+09 \quad 1.66 \mathrm{E}+09 \quad 1.77 \mathrm{E}+09 \quad 1.92 \mathrm{E}+092.14 \mathrm{E}+09 \quad 3.26 \mathrm{E}+09$ $9.43 \mathrm{E}+10$

$1.31 \mathrm{E}+072.44 \mathrm{E}+107.00 \mathrm{E}+10 \quad 1.50 \mathrm{E}+10 \quad 5.49 \mathrm{E}+10 \quad 5.44 \mathrm{E}+10 \quad 5.38 \mathrm{E}+101.28 \mathrm{E}+116.96 \mathrm{E}+101.57 \mathrm{E}+11$ $1.67 \mathrm{E}+11 \quad 2.21 \mathrm{E}+112.27 \mathrm{E}+11 \quad 1.03 \mathrm{E}+112.20 \mathrm{E}+11 \quad 2.20 \mathrm{E}+112.89 \mathrm{E}+11 \quad 9.23 \mathrm{E}+113.38 \mathrm{E}+11 \quad 8.83 \mathrm{E}+10$ $1.34 \mathrm{E}+102.65 \mathrm{E}+083.04 \mathrm{E}+06$ 
Table B.6. Calculated neutron and gamma fluxes normalized to the reactor power of $85 \mathrm{MW}$ for location K3, P10 (DOS-5).

Units are $\left(\mathrm{cm}^{-2} \mathrm{~s}^{-1}\right)$.

\begin{abstract}
DOS5-1
$1.46 \mathrm{E}+06$ 3.93E+06 1.05E+07 1.64E+07 3.63E+07 5.39E+07 4.96E+07 6.88E+07 9.83E+07 3.42E+07 $3.51 \mathrm{E}+076.58 \mathrm{E}+07 \quad 1.70 \mathrm{E}+08 \quad 1.94 \mathrm{E}+08 \quad 2.16 \mathrm{E}+08 \quad 1.97 \mathrm{E}+08 \quad 2.38 \mathrm{E}+08 \quad 2.65 \mathrm{E}+081.77 \mathrm{E}+08 \quad 5.37 \mathrm{E}+07$ $2.07 \mathrm{E}+08 \quad 2.53 \mathrm{E}+08$ 1.32E+07 4.67E+06 9.09E+06 5.95E+07 1.70E+08 $1.45 \mathrm{E}+081.45 \mathrm{E}+081.38 \mathrm{E}+08$ $2.07 \mathrm{E}+08 \quad 2.38 \mathrm{E}+08 \quad 3.68 \mathrm{E}+072.06 \mathrm{E}+08 \quad 5.44 \mathrm{E}+07 \quad 1.50 \mathrm{E}+07 \quad 3.27 \mathrm{E}+07 \quad 5.29 \mathrm{E}+072.87 \mathrm{E}+071.95 \mathrm{E}+08$ $2.24 \mathrm{E}+08 \quad 2.07 \mathrm{E}+08 \quad 1.75 \mathrm{E}+08 \quad 8.98 \mathrm{E}+07 \quad 6.20 \mathrm{E}+07 \quad 5.59 \mathrm{E}+07 \quad 4.92 \mathrm{E}+07 \quad 2.30 \mathrm{E}+08 \quad 2.24 \mathrm{E}+082.34 \mathrm{E}+08$ $2.26 \mathrm{E}+08 \quad 2.45 \mathrm{E}+08 \quad 2.52 \mathrm{E}+08 \quad 3.95 \mathrm{E}+08$ 4.15E+08 4.37E+08 4.57E+08 4.86E+08 5.33E+08 7.98E+08 $1.67 \mathrm{E}+10$

$1.74 \mathrm{E}+07$ 9.11E+09 4.71E+10 8.16E+09 $6.48 \mathrm{E}+10 \quad 5.17 \mathrm{E}+10 \quad 5.56 \mathrm{E}+101.28 \mathrm{E}+117.28 \mathrm{E}+10 \quad 2.03 \mathrm{E}+11$ $1.97 \mathrm{E}+112.44 \mathrm{E}+112.42 \mathrm{E}+11 \quad 1.07 \mathrm{E}+112.10 \mathrm{E}+112.26 \mathrm{E}+112.92 \mathrm{E}+119.79 \mathrm{E}+113.71 \mathrm{E}+11 \quad 1.07 \mathrm{E}+11$ $2.31 \mathrm{E}+104.50 \mathrm{E}+086.35 \mathrm{E}+05$
\end{abstract}

\title{
DOS5-2
}

$1.68 \mathrm{E}+064.55 \mathrm{E}+061.35 \mathrm{E}+072.00 \mathrm{E}+074.86 \mathrm{E}+07$ 7.29E+07 6.96E+07 9.83E+07 1.40E+08 5.01E+07 $5.17 \mathrm{E}+07 \quad 9.71 \mathrm{E}+07 \quad 2.58 \mathrm{E}+08 \quad 2.99 \mathrm{E}+08 \quad 3.33 \mathrm{E}+08 \quad 3.12 \mathrm{E}+08 \quad 3.69 \mathrm{E}+08 \quad 4.00 \mathrm{E}+08 \quad 2.67 \mathrm{E}+08 \quad 8.17 \mathrm{E}+07$ $3.23 \mathrm{E}+08 \quad 3.89 \mathrm{E}+08 \quad 2.00 \mathrm{E}+077.15 \mathrm{E}+06 \quad 1.39 \mathrm{E}+079.18 \mathrm{E}+072.65 \mathrm{E}+082.25 \mathrm{E}+082.25 \mathrm{E}+082.16 \mathrm{E}+08$ $3.21 \mathrm{E}+083.75 \mathrm{E}+08$ 5.78E+07 3.23E+08 8.57E+07 2.35E+07 5.05E+07 8.34E+07 4.66E+07 3.09E+08 $3.58 \mathrm{E}+083.30 \mathrm{E}+08 \quad 2.80 \mathrm{E}+08 \quad 1.46 \mathrm{E}+08 \quad 1.02 \mathrm{E}+08 \quad 9.11 \mathrm{E}+07 \quad 8.03 \mathrm{E}+073.83 \mathrm{E}+083.73 \mathrm{E}+083.94 \mathrm{E}+08$ $3.77 \mathrm{E}+084.20 \mathrm{E}+08 \quad 4.37 \mathrm{E}+086.89 \mathrm{E}+08$ 7.36E+08 7.85E+08 8.32E+08 $8.86 \mathrm{E}+089.79 \mathrm{E}+08 \quad 1.49 \mathrm{E}+09$ $3.23 \mathrm{E}+10$

$1.54 \mathrm{E}+071.31 \mathrm{E}+104.96 \mathrm{E}+109.65 \mathrm{E}+095.89 \mathrm{E}+104.99 \mathrm{E}+104.94 \mathrm{E}+101.21 \mathrm{E}+116.58 \mathrm{E}+101.80 \mathrm{E}+11$ $1.80 \mathrm{E}+112.23 \mathrm{E}+11 \quad 2.29 \mathrm{E}+11 \quad 1.02 \mathrm{E}+11 \quad 2.02 \mathrm{E}+11 \quad 2.17 \mathrm{E}+112.82 \mathrm{E}+119.46 \mathrm{E}+11 \quad 3.68 \mathrm{E}+11 \quad 1.05 \mathrm{E}+11$ $2.00 \mathrm{E}+103.43 \mathrm{E}+081.11 \mathrm{E}+06$

\section{DOS5-3}

$1.88 \mathrm{E}+06 \quad 5.21 \mathrm{E}+06 \quad 1.55 \mathrm{E}+072.40 \mathrm{E}+075.99 \mathrm{E}+079.45 \mathrm{E}+079.18 \mathrm{E}+071.34 \mathrm{E}+08 \quad 1.90 \mathrm{E}+086.75 \mathrm{E}+07$ $6.98 \mathrm{E}+07 \quad 1.33 \mathrm{E}+08 \quad 3.61 \mathrm{E}+08 \quad 4.21 \mathrm{E}+08 \quad 4.60 \mathrm{E}+08 \quad 4.35 \mathrm{E}+08 \quad 5.14 \mathrm{E}+08 \quad 5.41 \mathrm{E}+08 \quad 3.58 \mathrm{E}+08 \quad 1.12 \mathrm{E}+08$ $4.47 \mathrm{E}+08 \quad 5.28 \mathrm{E}+08 \quad 2.65 \mathrm{E}+07 \quad 9.46 \mathrm{E}+061.86 \mathrm{E}+07 \quad 1.28 \mathrm{E}+083.63 \mathrm{E}+08 \quad 3.16 \mathrm{E}+083.05 \mathrm{E}+083.03 \mathrm{E}+08$ $4.36 \mathrm{E}+08$ 5.25E+08 8.13E+07 4.44E+08 1.29E+08 3.60E+07 6.79E+07 1.07E+08 6.31E+07 4.37E+08 $5.17 \mathrm{E}+08 \quad 4.81 \mathrm{E}+08 \quad 4.12 \mathrm{E}+08 \quad 2.18 \mathrm{E}+08 \quad 1.52 \mathrm{E}+08 \quad 1.39 \mathrm{E}+08 \quad 1.18 \mathrm{E}+08 \quad 5.77 \mathrm{E}+08 \quad 5.68 \mathrm{E}+08 \quad 6.02 \mathrm{E}+08$ $5.89 \mathrm{E}+08 \quad 6.59 \mathrm{E}+08 \quad 6.88 \mathrm{E}+08 \quad 1.10 \mathrm{E}+09 \quad 1.18 \mathrm{E}+09 \quad 1.26 \mathrm{E}+091.34 \mathrm{E}+09 \quad 1.43 \mathrm{E}+09 \quad 1.61 \mathrm{E}+09 \quad 2.45 \mathrm{E}+09$ $6.73 \mathrm{E}+10$ $1.54 \mathrm{E}+071.86 \mathrm{E}+10 \quad 5.79 \mathrm{E}+101.23 \mathrm{E}+10 \quad 6.05 \mathrm{E}+10 \quad 5.37 \mathrm{E}+10 \quad 5.07 \mathrm{E}+101.26 \mathrm{E}+116.81 \mathrm{E}+101.82 \mathrm{E}+11$ $1.84 \mathrm{E}+112.24 \mathrm{E}+11 \quad 2.33 \mathrm{E}+11 \quad 1.05 \mathrm{E}+112.10 \mathrm{E}+112.23 \mathrm{E}+112.92 \mathrm{E}+119.78 \mathrm{E}+11 \quad 3.95 \mathrm{E}+11 \quad 1.21 \mathrm{E}+11$ $2.41 \mathrm{E}+104.74 \mathrm{E}+082.26 \mathrm{E}+06$ 
Table B.7. Calculated neutron and gamma fluxes normalized to the reactor power of $85 \mathrm{MW}$ for location $\mathrm{K} 7, \mathrm{P1}$ (DOS-6).

Units are $\left(\mathrm{cm}^{-2} \mathrm{~s}^{-1}\right)$.

\section{DOS6}

7.94E+05 1.82E+06 2.96E+06 4.68E+06 6.06E+06 6.42E+06 4.79E+06 4.62E+06 5.18E+06 1.42E+06 $1.29 \mathrm{E}+062.13 \mathrm{E}+064.00 \mathrm{E}+063.71 \mathrm{E}+063.49 \mathrm{E}+062.75 \mathrm{E}+063.29 \mathrm{E}+062.92 \mathrm{E}+061.97 \mathrm{E}+066.09 \mathrm{E}+05$

$2.38 \mathrm{E}+062.62 \mathrm{E}+061.17 \mathrm{E}+054.30 \mathrm{E}+049.01 \mathrm{E}+047.06 \mathrm{E}+051.76 \mathrm{E}+061.58 \mathrm{E}+061.45 \mathrm{E}+061.34 \mathrm{E}+06$ $2.13 \mathrm{E}+062.29 \mathrm{E}+06 \quad 3.83 \mathrm{E}+05 \quad 2.04 \mathrm{E}+06 \quad 8.45 \mathrm{E}+052.42 \mathrm{E}+05 \quad 1.59 \mathrm{E}+052.24 \mathrm{E}+052.21 \mathrm{E}+051.93 \mathrm{E}+06$ $2.07 \mathrm{E}+062.02 \mathrm{E}+06 \quad 1.59 \mathrm{E}+06 \quad 7.91 \mathrm{E}+05 \quad 5.92 \mathrm{E}+05 \quad 5.91 \mathrm{E}+05 \quad 3.94 \mathrm{E}+051.96 \mathrm{E}+061.96 \mathrm{E}+061.96 \mathrm{E}+06$ $1.96 \mathrm{E}+061.96 \mathrm{E}+061.96 \mathrm{E}+062.94 \mathrm{E}+062.94 \mathrm{E}+062.95 \mathrm{E}+062.96 \mathrm{E}+063.01 \mathrm{E}+063.20 \mathrm{E}+064.74 \mathrm{E}+06$ $2.43 \mathrm{E}+08$

$2.10 \mathrm{E}+077.64 \mathrm{E}+082.88 \mathrm{E}+10 \quad 5.14 \mathrm{E}+097.71 \mathrm{E}+104.92 \mathrm{E}+10 \quad 8.43 \mathrm{E}+101.49 \mathrm{E}+11 \quad 1.03 \mathrm{E}+112.30 \mathrm{E}+11$ $2.30 \mathrm{E}+113.38 \mathrm{E}+113.15 \mathrm{E}+11 \quad 1.39 \mathrm{E}+112.05 \mathrm{E}+112.72 \mathrm{E}+11 \quad 3.65 \mathrm{E}+11 \quad 1.38 \mathrm{E}+129.52 \mathrm{E}+119.35 \mathrm{E}+11$ $1.03 \mathrm{E}+121.84 \mathrm{E}+116.95 \mathrm{E}+08$

Table B.8. Calculated neutron and gamma fluxes normalized to the reactor power of $85 \mathrm{MW}$ for locations $\mathrm{K} 2 \mathrm{C}, \mathrm{P1}$

(DOS-8), and K2C, P4 ( DOS-9).

Units are $\left(\mathrm{cm}^{-2} \mathrm{~s}^{-1}\right)$.

\section{DOS8 and DOS9}

$2.28 \mathrm{E}+07$ 9.18E+07 2.57E+08 6.48E+08 1.35E+09 2.30E+09 3.19E+09 3.93E+09 4.99E+09 1.75E+09 $1.81 \mathrm{E}+094.07 \mathrm{E}+091.11 \mathrm{E}+10 \quad 1.25 \mathrm{E}+101.28 \mathrm{E}+101.19 \mathrm{E}+101.33 \mathrm{E}+101.34 \mathrm{E}+10 \quad 8.59 \mathrm{E}+092.77 \mathrm{E}+09$ $1.18 \mathrm{E}+10 \quad 1.38 \mathrm{E}+10 \quad 6.73 \mathrm{E}+08 \quad 2.40 \mathrm{E}+08 \quad 4.66 \mathrm{E}+08 \quad 3.25 \mathrm{E}+09 \quad 9.52 \mathrm{E}+09 \quad 8.67 \mathrm{E}+09 \quad 8.19 \mathrm{E}+09 \quad 8.84 \mathrm{E}+09$ $1.18 \mathrm{E}+10 \quad 1.52 \mathrm{E}+10 \quad 2.28 \mathrm{E}+09 \quad 1.26 \mathrm{E}+103.53 \mathrm{E}+09 \quad 9.69 \mathrm{E}+08 \quad 2.22 \mathrm{E}+09 \quad 3.72 \mathrm{E}+09 \quad 2.01 \mathrm{E}+09 \quad 1.24 \mathrm{E}+10$ $1.56 \mathrm{E}+10 \quad 1.53 \mathrm{E}+10 \quad 1.37 \mathrm{E}+10 \quad 7.35 \mathrm{E}+094.99 \mathrm{E}+094.46 \mathrm{E}+094.15 \mathrm{E}+091.97 \mathrm{E}+101.90 \mathrm{E}+102.06 \mathrm{E}+10$ $2.12 \mathrm{E}+10 \quad 2.35 \mathrm{E}+10 \quad 2.44 \mathrm{E}+10 \quad 3.88 \mathrm{E}+10 \quad 4.12 \mathrm{E}+10 \quad 4.37 \mathrm{E}+10 \quad 4.58 \mathrm{E}+10 \quad 4.85 \mathrm{E}+10 \quad 5.31 \mathrm{E}+10 \quad 7.87 \mathrm{E}+10$ $2.75 \mathrm{E}+12$

$1.94 \mathrm{E}+07 \quad 2.24 \mathrm{E}+11 \quad 5.63 \mathrm{E}+11 \quad 1.18 \mathrm{E}+112.58 \mathrm{E}+112.68 \mathrm{E}+114.18 \mathrm{E}+11 \quad 5.36 \mathrm{E}+113.37 \mathrm{E}+11 \quad 1.03 \mathrm{E}+12$ $6.38 \mathrm{E}+118.30 \mathrm{E}+11 \quad 8.59 \mathrm{E}+113.96 \mathrm{E}+119.95 \mathrm{E}+11 \quad 8.59 \mathrm{E}+11 \quad 1.20 \mathrm{E}+12 \quad 3.92 \mathrm{E}+12 \quad 1.45 \mathrm{E}+12 \quad 3.82 \mathrm{E}+11$ $4.35 \mathrm{E}+102.21 \mathrm{E}+098.67 \mathrm{E}+07$

Table B.9. Calculated neutron and gamma fluxes normalized to the reactor power of $85 \mathrm{MW}$ for locations 2D1W, 2D2W, and 2D3W.

Units are $\left(\mathrm{cm}^{-2} \mathrm{~s}^{-1}\right)$.

\section{D1W, 2D2W, and 2D3W}

$1.33 \mathrm{E}+06$ 3.87E+06 8.31E+06 1.67E+07 2.97E+07 4.56E+07 4.97E+07 6.20E+07 8.93E+07 2.99E+07 $2.97 \mathrm{E}+07 \quad 5.43 \mathrm{E}+07 \quad 1.27 \mathrm{E}+08 \quad 1.38 \mathrm{E}+08 \quad 1.67 \mathrm{E}+08 \quad 1.68 \mathrm{E}+08 \quad 1.96 \mathrm{E}+08 \quad 2.62 \mathrm{E}+08 \quad 1.87 \mathrm{E}+08 \quad 5.38 \mathrm{E}+07$ $1.97 \mathrm{E}+08 \quad 2.80 \mathrm{E}+08 \quad 1.73 \mathrm{E}+076.15 \mathrm{E}+06 \quad 1.16 \mathrm{E}+07 \quad 6.44 \mathrm{E}+071.78 \mathrm{E}+08 \quad 1.39 \mathrm{E}+081.43 \mathrm{E}+08 \quad 1.39 \mathrm{E}+08$ $2.04 \mathrm{E}+08 \quad 2.35 \mathrm{E}+08 \quad 3.72 \mathrm{E}+07 \quad 1.95 \mathrm{E}+08$ 7.20E+07 $2.06 \mathrm{E}+07 \quad 1.59 \mathrm{E}+07 \quad 3.27 \mathrm{E}+073.05 \mathrm{E}+07 \quad 1.84 \mathrm{E}+08$ $2.04 \mathrm{E}+08 \quad 1.90 \mathrm{E}+08 \quad 1.50 \mathrm{E}+08 \quad 7.65 \mathrm{E}+07 \quad 5.61 \mathrm{E}+07 \quad 5.43 \mathrm{E}+07 \quad 3.76 \mathrm{E}+07 \quad 1.91 \mathrm{E}+08 \quad 1.85 \mathrm{E}+08 \quad 1.85 \mathrm{E}+08$ $1.80 \mathrm{E}+08 \quad 1.82 \mathrm{E}+08 \quad 1.83 \mathrm{E}+08 \quad 2.75 \mathrm{E}+08 \quad 2.77 \mathrm{E}+08 \quad 2.77 \mathrm{E}+08 \quad 2.76 \mathrm{E}+08 \quad 2.77 \mathrm{E}+082.88 \mathrm{E}+08 \quad 4.08 \mathrm{E}+08$ $1.09 \mathrm{E}+10$

$1.34 \mathrm{E}+07$ 7.73E+09 5.03E+10 7.73E+09 5.66E+10 4.55E+10 8.35E+10 $1.27 \mathrm{E}+118.50 \mathrm{E}+102.12 \mathrm{E}+11$

$1.81 \mathrm{E}+112.52 \mathrm{E}+11 \quad 2.35 \mathrm{E}+11 \quad 1.06 \mathrm{E}+11 \quad 1.85 \mathrm{E}+11 \quad 2.22 \mathrm{E}+11 \quad 2.96 \mathrm{E}+11 \quad 1.04 \mathrm{E}+12 \quad 5.96 \mathrm{E}+11 \quad 4.45 \mathrm{E}+11$ $3.53 \mathrm{E}+115.33 \mathrm{E}+101.96 \mathrm{E}+08$ 
Table B10. Calculated neutron and gamma fluxes normalized to the reactor power of $85 \mathrm{MW}$ for locations 2D1C and 2D2C.

Units are $\left(\mathrm{cm}^{-2} \mathrm{~s}^{-1}\right)$.

\section{D1C and 2D2C}

1.36E+07 5.46E+07 1.56E+08 3.80E+08 7.73E+08 1.28E+09 1.61E+09 1.95E+09 2.43E+09 8.39E+08

8.39E+08 1.71E+09 4.29E+09 4.69E+09 4.71E+09 4.10E+09 4.98E+09 4.90E+09 3.30E+09 1.05E+09

4.15E+09 4.86E+09 2.34E+08 8.50E+07 1.73E+08 1.28E+09 3.37E+09 3.06E+09 2.88E+09 2.83E+09

4.33E+09 4.96E+09 8.20E+08 4.44E+09 1.80E+09 5.24E+08 3.96E+08 6.15E+08 5.53E+08 4.39E+09

4.97E+09 4.97E+09 4.08E+09 2.10E+09 1.57E+09 1.57E+09 1.09E+09 5.53E+09 5.70E+09 5.96E+09

$6.21 \mathrm{E}+096.52 \mathrm{E}+096.83 \mathrm{E}+09 \quad 1.09 \mathrm{E}+10 \quad 1.16 \mathrm{E}+10 \quad 1.24 \mathrm{E}+10 \quad 1.32 \mathrm{E}+10 \quad 1.43 \mathrm{E}+101.62 \mathrm{E}+102.52 \mathrm{E}+10$

$2.25 \mathrm{E}+12$

$1.09 \mathrm{E}+074.64 \mathrm{E}+10 \quad 1.91 \mathrm{E}+112.55 \mathrm{E}+10 \quad 9.86 \mathrm{E}+10 \quad 9.69 \mathrm{E}+10 \quad 1.94 \mathrm{E}+112.45 \mathrm{E}+11 \quad 1.62 \mathrm{E}+117.27 \mathrm{E}+11$

$2.99 \mathrm{E}+114.22 \mathrm{E}+11 \quad 4.14 \mathrm{E}+11 \quad 1.90 \mathrm{E}+11 \quad 3.76 \mathrm{E}+11 \quad 4.14 \mathrm{E}+11 \quad 5.74 \mathrm{E}+112.11 \mathrm{E}+121.26 \mathrm{E}+129.18 \mathrm{E}+11$

$5.47 \mathrm{E}+117.51 \mathrm{E}+102.74 \mathrm{E}+08$ 



\section{APPENDIX C}

COMPARISON OF ADJUSTED IRRADIATION PARAMETERS FROM DIFFERENT ADJUSTMENT RUNS AND ADDITIONAL CONSIDERATIONS 

In the determination of the adjusted irradiation parameters, several measured reaction rates were not used because their reaction rates were inconsistent with other measurements. A complete list of omitted measurements is provided in Table 18. Here, we will investigate the impact of the omitted measurements on the adjusted irradiation parameters. The spectrum adjustment calculations and adjusted irradiation parameter values reported in Tables 4-17 are referred to in the following as the "reference runs" and "reference irradiation parameters," respectively, because they were chosen to be the best estimate of the irradiation parameters.

We will concentrate on the dosimeter locations where two or more dosimeter measurements were omitted. Because Ce-141 was not used in any of the adjustment calculations, the locations where only Ce-141 was omitted will not be discussed. Likewise, locations where both Ce-141 and nondiluted cobalt measurements were omitted will not be discussed because the information from nondiluted cobalt dosimeters is redundant (and less accurate) if the activity of a diluted cobalt dosimeter is available. The remaining five locations that need to be addressed are $\mathrm{K} 3, \mathrm{P} 8 ; \mathrm{K} 2 \mathrm{C}$, $\mathrm{P} 1$; K2C, P4; 2D2C; and 2D1C.

At the K3, P8 location (DOS-4) all (3) neptunium measurements and all (2) copper measurements were not used in the reference adjustment run reported in Table 8. When the $\mathrm{Zr}-95$, Ru-103, and Cs-137 reaction rates are used in the adjustments, the fast neutron flux $(\mathrm{E}>1 \mathrm{MeV})$ at position 2 is reduced by $4 \%$ and the total (neutron + gamma) dpa rate is decreased by $6 \%$. When the two copper measurements are included (in addition to the neptunium fission products), the fast flux is $8 \%$ smaller and the total $\mathrm{dpa} / \mathrm{s}$ is $7 \%$ smaller than the values obtained from the reference adjustment run (performed without those dosimeters). The $\chi^{2}$ per degree of freedom increased considerably when the neptunium and copper dosimeters were used, and the increase was particularly large when the two copper measurements were added. Detailed comparison of the three adjustment runs are shown in Table C.1.

The equivalent comparison for the $\mathrm{K} 2 \mathrm{C}$, $\mathrm{P} 1$ location (DOS-8) is given in Table C.2. When neptunium fission products were included in the adjustment, the adjusted fast neutron flux and total dpa rate were $6 \%$ smaller than the values obtained in the reference run. When the two copper dosimeters were added, the reductions in fast neutron flux and total dpa rate were $9 \%$ and $7 \%$, respectively. Again a marked increase in the $\chi^{2}$ per degree of freedom was observed.

Table C.3 gives the comparison of adjustment runs for the K2C, P4 location (DOS-9). Here the adjustment with Zr-95, Ru-103, and Cs-137 activities lowered the fast flux and total dpa rate by $8 \%$ and $7 \%$, respectively; adding the two copper measurements gave a $13 \%$ reduction in fast flux and a $10 \%$ reduction in the total dpa rate with respect to the values from the reference adjustment run. In this case, the increase in the $\chi^{2}$ per degree of freedom is somewhat smaller than in the previous two cases; however, it is still quite large when the two copper measurements are added.

At the location 2D2C, the copper measurement was eliminated, as well as Ru-103. Adding the copper, or adding copper and Ru-103 dramatically increased the value of $\chi^{2}$ per degree of freedom, while it decreased the fast flux and the total dpa rate by less than $4 \%$. Finally, an adjustment with all neptunium measurements eliminated was performed. The irradiation parameters remained almost unchanged; however, the $\chi^{2}$ per degree of freedom was substantially higher than the value in the reference adjustment run. A comparison is provided in Table C.4.

In Table C.5, a comparison of the adjusted irradiation parameters for position $2 \mathrm{D} 1 \mathrm{C}$ is provided. In the reference adjustment five activation measurements were not used: copper, two neptunium fission products (Ce-141 and $\mathrm{Ru}-103$ ), and two nondiluted cobalt measurements, one under gadolinium cover and the other bare. As was already discussed the omission of cobalt 
measurements is of no importance because the diluted cobalt dosimeters were also measured under gadolinium cover and bare and were used in the adjustment. The effect of including the copper dosimeter in the adjustment is $\sim 5 \%$ reduction in neutron flux above $1 \mathrm{MeV}$ and $\sim 1 \%$ reduction of total dpa. The value of $\chi^{2}$ per degree of freedom increases to 1.235 . When both copper and Ru-103 are included in the adjustment, the $\chi 2$ per degree of freedom increases to 2.067 , while the neutron flux above $1 \mathrm{MeV}$ increases by $1 \%$, and the total dpa increases by $4 \%$. Therefore, while omitting these two dosimeters improves the consistency of the data, the effect on the adjusted irradiation parameters is quite small and well within the uncertainty of one standard deviation of the adjusted irradiation parameters. For the Ce-141 activity, the calculated/measured value was $\sim 2$ and was well out of the line of the calculated/measured values for the other neptunium fission products and was omitted from the adjustment for this reason. The last column on the right demonstrates the effect of eliminating all measurements of the neptunium fission products. This results in a slight increase in the irradiation parameters; however, the increases are smaller than the standard deviations.

In summary, the effect of the dosimeters omitted from the adjustment calculations is typically limited to changes in the fast neutron flux and total dpa rate of $\sim 7$ to $9 \%$ and reaches a maximum of $10 \%$ to $13 \%$ at one location. The largest of these changes is in the range of from $1 \times \sigma$ to $1.5 \times \sigma$, where $1-\sigma$ denotes the uncertainty in the adjusted irradiation parameters. Further, at all but one location the omission of the selected dosimeters from the adjustment calculation resulted in higher values for the irradiation parameters.

The locations $2 \mathrm{D} 1 \mathrm{C}$ and $2 \mathrm{D} 2 \mathrm{C}$ are of particular interest because they correspond to the corner of the nozzle of beam tube 2, where the beam tube was enlarged. Of all the locations where the dosimeters were placed, these two locations are in the most intense neutron and gamma-ray field with the exception of Key $2 \mathrm{C}$. The two locations are symmetric with respect to the beam tube, which is pointing directly at the center of the core. From symmetry considerations, it would be expected that the irradiation parameters at the two locations will be quite similar. The transport calculations, which provided the calculated neutron flux and gamma-ray values, were performed with a cylindrical R-Z model, and consequently predicted identical fluxes at both locations, as can be observed from the calculated values in Tables 16 and 17, respectively. A more detailed threedimensional Monte Carlo calculation of the Key 2 location provided fluxes at 2D1C and 2D2C, which agreed within $\sim 5 \%$. However, the adjusted fast neutron flux at location $2 \mathrm{D} 2 \mathrm{C}$ is $\sim 34 \%$ higher than the flux at $2 \mathrm{D} 1 \mathrm{C}$, and the adjusted total dpa rate is $\sim 29 \%$ higher at location $2 \mathrm{D} 2 \mathrm{C}$ than at the location 2D1C. Detailed comparison of the adjusted irradiation parameters in the tubular dosimeter capsules around Key 2 is given in Fig. C.1.

In Table C.6, the measurements performed in locations 2D1C and 2D2C during HFIR fuel cycles 400 and 401 are compared. Because the measured specific activities of the dosimeters are compared, there is no possibility of interference from the analysis and/or calculations. The measurements clearly indicate that the radiation field at the location 2D2C is considerably higher than at the location 2D1C. Comparison of the calculated reaction rates and "measured" reaction rates deduced from the measured specific activities is given in the Table C.7. The calculated reaction rates were obtained from the two-dimensional $\mathrm{R}-\mathrm{Z}$ calculations and are consequently the same at locations 2D2C and 2D1C; however, the measurements indicate that the adjustment will tend to increase the irradiation parameters at location $2 \mathrm{D} 2 \mathrm{C}$ and decrease at location $2 \mathrm{D} 1 \mathrm{C}$.

Figure C. 1 shows that a similar-albeit smaller-increase in irradiation parameters is observed between locations 2D1W, 2D2W, and 2D3W, which are the locations on the concentric, but larger ring around beam tube 2 . This indicates that the measurements show a considerable increase in the neutron and gamma field from the "top" (or 12 o'clock) location to the core 
midplane (or 3 o'clock) location around the beam line, something that was not revealed by the calculations. Because the HFIR core is only $\sim 60 \mathrm{~cm}$ tall, and the nozzle has the inner radius of $\sim 18 \mathrm{~cm}$, the 12 o'clock location of the nozzle is actually closer to the top of the core than to the midplane. The decrease of the core power with the distance from the core midplane may play a role in the observed flux changes. (It is interesting to observe that the 12 o'clock weld location is approximately $5 \mathrm{~cm}$ above the top of the core.)

While the adjusted neutron flux is higher at location 2D2C than at the location $2 \mathrm{D} 1 \mathrm{C}$, the gammaray flux is only about one-half of the value at location $2 \mathrm{D} 1 \mathrm{C}$, which seems to be inconsistent. However, the total dpa rate at 2D2C and 2D1C is dominated by the neutron-induced dpa rate, which makes the inconsistencies in gamma-induced dpa rate less important. Given the relatively large differences in the measured-to-calculated reaction rate ratios between the beryllium and neptunium dosimeters and even between the two nickel dosimeters at location 2D1C, it would be of benefit to repeat the measurements at least at these two nozzle-corner locations and try to verify or eliminate the observed differences. As stated earlier, the accuracy of the dosimeter locations is very important because there is a steep decrease in the radiation field around the beam line. From the beam line nozzle corner to the beam line nozzle weld the fast neutron flux decreases on average by factor of 2 every $3.60 \mathrm{~cm}$. A small deviation - of the order of $10 \mathrm{~mm}-$ of the actual location of the dosimeters during irradiation from the locations assumed in the analysis could be responsible for the observed variations in the measured reaction rates. For example, if the tubular capsule in location 2D1 had remained $\sim 10 \mathrm{~mm}$ above the designated location during the irradiation, it would have caused all the unexpected variations in the measurements around beam tube 2 .

To further address the concerns about not using copper dosimeters in the flux adjustment calculations at certain locations, the following was done. For location 2D1C (capsule HRB-1C), a new estimate of the "calculated" neutron fluxes was created by replacing the values in the five highest energy groups by the values obtained from the adjustment run with all dosimeters (including copper). The corresponding flux values are given in Table C.8 and are considerably higher than the values from the transport calculation. The neutron fluxes for all other groups as well as the gamma-ray fluxes were retained unchanged (i.e., they were obtained from the transport calculations). With these "new" fluxes the adjustment was repeated, and the copper dosimeter measurement was taken into account in this adjustment calculation. Table C.9 compares results from this run with results reported for location 2D1C (given in Table 17, in which the copper dosimeter was not used). The changes are of the order of 3-7\% and are considerably smaller than the reported standard deviations of the adjusted irradiation parameters. This shows that increasing the neutron fluxes in the five highest-energy neutron groups to the values consistent with the measured copper reaction rates would not have a substantial effect on the adjusted irradiation parameters. It also further illustrates that eliminating copper dosimeters from the adjustment did not introduce bias in the adjusted results, as was also shown in Table C.5. 
Table C.1. Comparison of the adjusted irradiation parameters for capsule DOS-4. The last two columns on the right side give the ratios of the irradiation parameters obtained from adjustments, which took into account the neptunium dosimeter and the neptunium and the two copper dosimeters, respectively, to the reference adjusted values (from Table 8; given in column 3), which were obtained without neptunium and copper dosimeters

\begin{tabular}{|c|c|c|c|c|c|c|c|}
\hline & \multirow[b]{2}{*}{ Irradiation parameter } & \multirow{2}{*}{$\begin{array}{c}\text { Reference } \\
\text { adjusted } \\
\text { value } \\
()^{\mathrm{a}}\end{array}$} & \multirow[b]{2}{*}{$\begin{array}{l}\text { Std. }^{b} \\
(\%)\end{array}$} & \multirow[b]{2}{*}{$\begin{array}{l}\text { Adj./ } \\
\text { calc. }\end{array}$} & \multirow[b]{2}{*}{$\begin{array}{c}\text { Ni58(n,p) } \\
\text { M/C }\end{array}$} & \multicolumn{2}{|c|}{ Ratio } \\
\hline & & & & & & $\begin{array}{l}\text { Np meas. included } \\
\text { (Zr-95, Ru-103, } \\
\text { and Cs-137) }\end{array}$ & $\begin{array}{c}\text { Np and two Cu } \\
\text { measurements } \\
\text { included }\end{array}$ \\
\hline \multirow{6}{*}{ Position 1} & Neutron flux $(E>1 \mathrm{MeV})$ & $1.76 \mathrm{E}+09$ & 7 & 0.96 & 0.97 & 0.97 & 0.96 \\
\hline & Neutron flux $(E>0.5 \mathrm{MeV})$ & $2.85 \mathrm{E}+09$ & 8 & 0.95 & & 0.97 & 0.96 \\
\hline & Neutron flux $(E>0.1 \mathrm{MeV})$ & $4.48 \mathrm{E}+09$ & 8 & 0.94 & & 0.96 & 0.95 \\
\hline & Neutron flux $(\mathrm{E}<0.414 \mathrm{eV})$ & $1.94 \mathrm{E}+10$ & 6 & 0.62 & & 1.00 & 1.00 \\
\hline & $\mathrm{dpa} / \mathrm{s}(\mathrm{Fe}, \mathrm{ASTM})$ & $2.86 \mathrm{E}-12$ & 6 & 0.92 & & 0.98 & 0.98 \\
\hline & $\mathrm{dpa} / \mathrm{s}(\mathrm{Fe}, \mathrm{ENDF}-\mathrm{VI})$ & $2.84 \mathrm{E}-12$ & 6 & 0.92 & & 0.98 & 0.98 \\
\hline \multirow{9}{*}{ Position 2} & Neutron flux $(\mathrm{E}>1 \mathrm{MeV})$ & $2.64 \mathrm{E}+09$ & 8 & 1.14 & 1.16 & 0.96 & 0.92 \\
\hline & Neutron flux $(\mathrm{E}>0.5 \mathrm{MeV})$ & $4.45 \mathrm{E}+09$ & 8 & 1.14 & & 0.95 & 0.92 \\
\hline & Neutron flux $(E>0.1 \mathrm{MeV})$ & $7.19 \mathrm{E}+09$ & 9 & 1.13 & & 0.95 & 0.92 \\
\hline & Neutron flux $(\mathrm{E}<0.414 \mathrm{eV})$ & $3.62 \mathrm{E}+10$ & 22 & 0.85 & & 1.00 & 0.94 \\
\hline & $\mathrm{dpa} / \mathrm{s}(\mathrm{Fe}, \mathrm{ASTM})$ & $4.38 \mathrm{E}-12$ & 7 & 1.10 & & 0.96 & 0.94 \\
\hline & $\mathrm{dpa} / \mathrm{s}(\mathrm{Fe}, \mathrm{ENDF}-\mathrm{VI})$ & $4.35 \mathrm{E}-12$ & 7 & 1.10 & & 0.96 & 0.94 \\
\hline & Gamma dpa/s & $1.05 \mathrm{E}-12$ & 12 & 1.06 & & 0.87 & 0.88 \\
\hline & Gamma flux & $3.33 \mathrm{E}+12$ & 18 & 1.05 & & 1.03 & 1.04 \\
\hline & Neutron + gamma dpa/s & $5.43 \mathrm{E}-12$ & 6 & 1.09 & & 0.94 & 0.93 \\
\hline \multirow{7}{*}{ Position 3} & Neutron flux $(\mathrm{E}>1 \mathrm{MeV})$ & $4.00 \mathrm{E}+09$ & 7 & 1.25 & 1.27 & 0.97 & 0.98 \\
\hline & Neutron flux $(E>0.5 \mathrm{MeV})$ & $6.72 \mathrm{E}+09$ & 8 & 1.25 & & 0.97 & 0.97 \\
\hline & Neutron flux $(E>0.1 \mathrm{MeV})$ & $1.09 \mathrm{E}+10$ & 8 & 1.24 & & 0.96 & 0.98 \\
\hline & Neutron flux $(\mathrm{E}<0.414 \mathrm{eV})$ & $1.13 \mathrm{E}+11$ & 6 & 1.20 & & 1.00 & 1.00 \\
\hline & $\mathrm{dpa} / \mathrm{s}(\mathrm{Fe}, \mathrm{ASTM})$ & $7.15 \mathrm{E}-12$ & 6 & 1.23 & & 0.98 & 0.99 \\
\hline & $\mathrm{dpa} / \mathrm{s}(\mathrm{Fe}, \mathrm{ENDF}-\mathrm{VI})$ & $7.06 \mathrm{E}-12$ & 6 & 1.23 & & 0.98 & 0.99 \\
\hline & $\chi^{2}$ per degree of freedom & 0.5724 & & & & 0.9370 & 2.312 \\
\hline
\end{tabular}

\footnotetext{
${ }^{a}$ Units are $\mathrm{cm}^{-2} \mathrm{~s}^{-1}$ for neutron and gamma-ray fluxes and $\mathrm{s}^{-1}$ for $\mathrm{dpa} / \mathrm{s}$.
}

${ }^{b}$ Percent standard deviation. 
Table C.2. Comparison of adjusted irradiation parameters for capsule DOS-8, at K2C, P1, for several adjustment runs

\begin{tabular}{|c|c|c|c|c|c|c|}
\hline \multirow[b]{2}{*}{ Irradiation parameter } & \multirow[b]{2}{*}{$\begin{array}{c}\text { Reference } \\
\text { adjusted } \\
\text { value } \\
()^{a}\end{array}$} & \multirow[b]{2}{*}{$\begin{array}{l}\text { Std. }^{b} \\
(\%)\end{array}$} & \multirow[b]{2}{*}{$\begin{array}{l}\text { Adj./ } \\
\text { calc. }\end{array}$} & \multirow[b]{2}{*}{$\begin{array}{l}\text { Ni58(n,p) } \\
\text { M/C }\end{array}$} & \multicolumn{2}{|c|}{ Ratio $^{c}$} \\
\hline & & & & & $\begin{array}{c}\text { Np meas. } \\
\text { Included } \\
\text { (Zr-95, } \\
\text { Ru-103, } \\
\text { and Cs-137) }\end{array}$ & $\begin{array}{c}\text { Np and } \\
\text { two Cu } \\
\text { meas. } \\
\text { included }\end{array}$ \\
\hline Neutron flux $(\mathrm{E}>1 \mathrm{MeV})$ & $1.01 \mathrm{E}+11$ & 6 & 1.51 & 1.51 & 0.94 & 0.91 \\
\hline Neutron flux $(\mathrm{E}>0.5 \mathrm{MeV})$ & $1.66 \mathrm{E}+11$ & 7 & 1.50 & 1.55 & 0.93 & 0.91 \\
\hline Neutron flux $(\mathrm{E}>0.1 \mathrm{MeV})$ & $2.71 \mathrm{E}+11$ & 8 & 1.48 & 1.51 & 0.93 & 0.90 \\
\hline Neutron flux $(\mathrm{E}<0.414 \mathrm{eV})$ & $2.60 \mathrm{E}+12$ & 5 & 0.95 & & 1.00 & 1.00 \\
\hline $\mathrm{dpa} / \mathrm{s}(\mathrm{Fe}, \mathrm{ASTM})$ & $1.73 \mathrm{E}-10$ & 5 & 1.38 & & 0.95 & 0.94 \\
\hline $\mathrm{dpa} / \mathrm{s} \quad(\mathrm{Fe}, \mathrm{ENDF}-\mathrm{VI})$ & $1.71 \mathrm{E}-10$ & 5 & 1.39 & & 0.95 & 0.94 \\
\hline Gamma dpa/s & $9.73 \mathrm{E}-12$ & 22 & 1.25 & & 0.79 & 0.80 \\
\hline Gamma flux & $1.90 \mathrm{E}+13$ & 24 & 1.24 & & 0.88 & 0.88 \\
\hline Neutron + gamma dpa/s & $1.83 \mathrm{E}-10$ & 5 & 1.37 & & 0.94 & 0.93 \\
\hline$\chi^{2}$ per degree of freedom & 0.4928 & & & & 0.9589 & 2.834 \\
\hline $\begin{array}{l}{ }^{a} \text { Units are } \mathrm{cm}^{-2} \mathrm{~s}^{-1} \text { for neut } \\
{ }^{b} \text { Percent standard deviatic } \\
{ }^{c} \text { Ratio of adjusted values } \\
\text { the adjustment (column 6), an } \\
\text { (column 7), to the reference a } \\
\text { measurements. The reference }\end{array}$ & $\begin{array}{l}\text { and gamma- } \\
\text { ained after ne } \\
\text { eptunium and }\end{array}$ & y fluxe & $\begin{array}{l}1 \mathrm{~s}^{-1} \text { for } \\
\text { suremer } \\
\text { er meas } \\
2 \text {, whi }\end{array}$ & $\begin{array}{l}\text { /s. } \\
\text { Zr-95, Ru- } \\
\text { ments were } \\
\text { vere obtain }\end{array}$ & $\begin{array}{l}\text {, and Cs-137) } \\
\text { ed in the adjus } \\
\text { without these }\end{array}$ & $\begin{array}{l}\text { ere used in } \\
\text { nent }\end{array}$ \\
\hline
\end{tabular}

Table C.3. Comparison of adjusted irradiation parameters for capsule DOS-9, at $\mathrm{K2C}$, P4, for several adjustment runs

\begin{tabular}{|c|c|c|c|c|c|c|}
\hline \multirow[b]{2}{*}{ Irradiation parameter } & \multirow[b]{2}{*}{$\begin{array}{l}\text { Reference } \\
\text { adjusted } \\
\text { value } \\
()^{a}\end{array}$} & \multirow[b]{2}{*}{$\begin{array}{l}\text { Std. }^{b} \\
(\%)\end{array}$} & \multirow[b]{2}{*}{$\begin{array}{l}\text { Adj./ } \\
\text { calc. }\end{array}$} & \multirow[b]{2}{*}{$\begin{array}{c}\text { Ni58(n,p) } \\
\text { M/C }\end{array}$} & \multicolumn{2}{|l|}{ Ratio $^{c}$} \\
\hline & & & & & $\begin{array}{c}\text { Np meas. included } \\
(\mathrm{Zr}-95, \\
\text { Ru-103, } \\
\text { and Cs-137) }\end{array}$ & $\begin{array}{c}\text { Np and } \\
\text { two Cu } \\
\text { meas. } \\
\text { included }\end{array}$ \\
\hline Neutron flux $(\mathrm{E}>1 \mathrm{MeV})$ & $9.97 \mathrm{E}+10$ & 9 & 1.48 & 1.44 & 0.92 & 0.87 \\
\hline Neutron flux $(\mathrm{E}>0.5 \mathrm{MeV})$ & $1.63 \mathrm{E}+11$ & 10 & 1.47 & 1.56 & 0.91 & 0.86 \\
\hline Neutron flux $(E>0.1 \mathrm{MeV})$ & $2.62 \mathrm{E}+11$ & 11 & 1.43 & 1.53 & 0.90 & 0.86 \\
\hline Neutron flux $(\mathrm{E}<0.414 \mathrm{eV})$ & $2.89 \mathrm{E}+12$ & 3 & 1.05 & & 1.00 & 1.00 \\
\hline $\mathrm{dpa} / \mathrm{s}(\mathrm{Fe}, \mathrm{ASTM})$ & $1.69 \mathrm{E}-10$ & 7 & 1.35 & & 0.94 & 0.91 \\
\hline $\mathrm{dpa} / \mathrm{s}(\mathrm{Fe}, \mathrm{ENDF}-\mathrm{VI})$ & $1.67 \mathrm{E}-10$ & 7 & 1.35 & & 0.93 & 0.90 \\
\hline Gamma dpa/s & $1.04 \mathrm{E}-11$ & 25 & 1.34 & & 0.81 & 0.85 \\
\hline Gamma flux & $1.99 \mathrm{E}+13$ & 30 & 1.30 & & 0.91 & 0.93 \\
\hline Neutron + gamma dpa/s & $1.80 \mathrm{E}-10$ & 6 & 1.35 & & 0.93 & 0.90 \\
\hline$\chi^{2}$ per degree of freedom & 0.9625 & & & & 1.044 & 1.786 \\
\hline
\end{tabular}

${ }^{\mathrm{a}}$ Units are $\mathrm{cm}^{-2} \mathrm{~s}^{-1}$ for neutron and gamma-ray fluxes and $\mathrm{s}^{-1}$ for $\mathrm{dpa} / \mathrm{s}$.

${ }^{b}$ Percent standard deviation.

${ }^{c}$ Ratio of adjusted values obtained after neptunium measurements (Zr-95, Ru-103, and Cs-137) were used in the adjustment (column 6), and neptunium and the two copper measurements were used in the adjustment (column 7), to the reference adjusted values (column 2) obtained without these measurements. The reference adjustment run is given in Table 12. 
Table C.4. Comparison of adjusted irradiation parameters at location 2D2C for different adjustment runs. The capsules were HRB2-2G and HRB2-2A, position 2; dosimeter sets GD-16 and AL-29 (HRB2-2C).

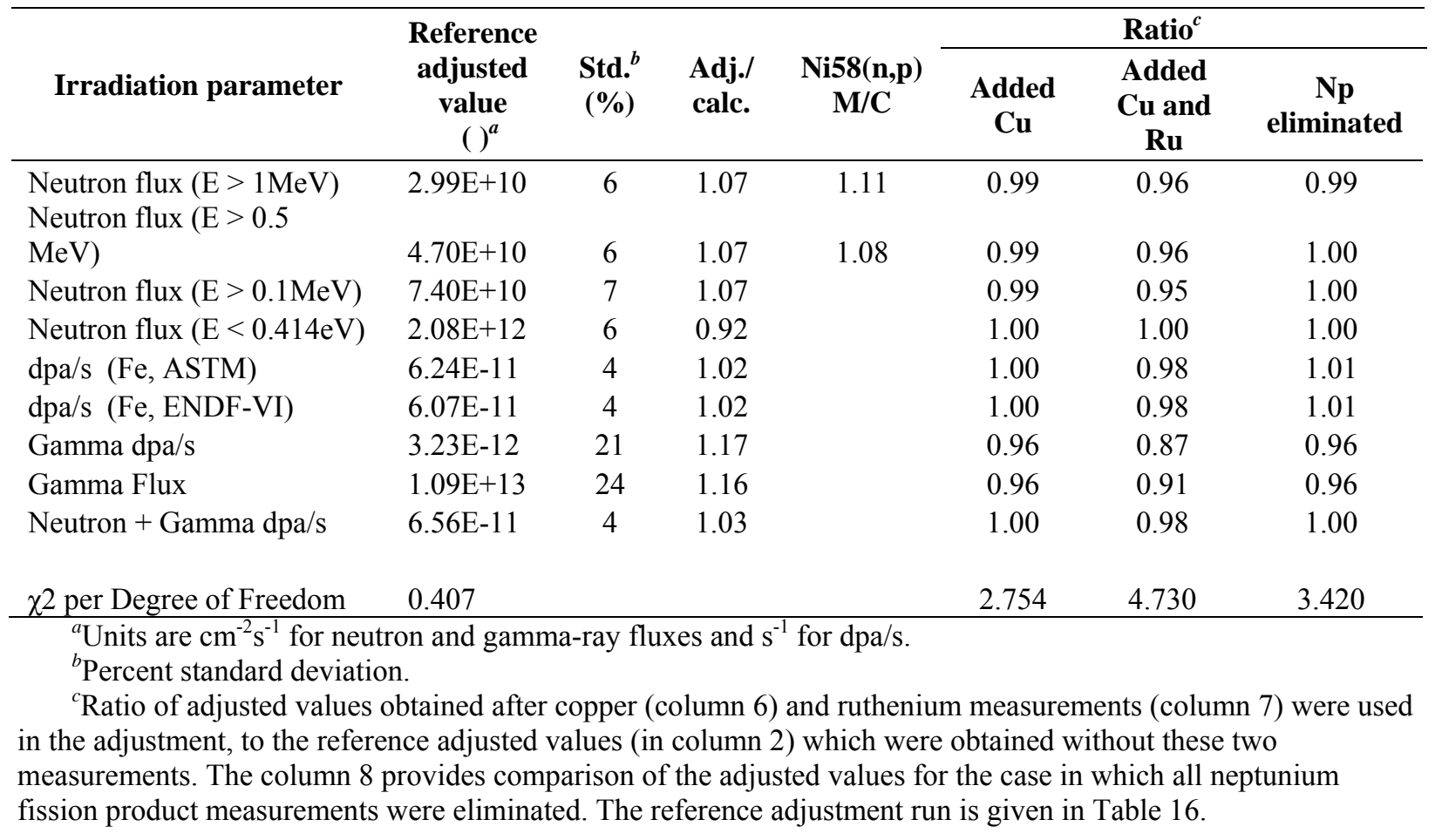

Table C.5. Comparison of adjusted irradiation parameters at location 2D1C for different adjustment runs. The capsules were HRB2-1G and HRB2-1A, position 2; dosimeter sets GD-14 and AL-27 (HRB2-1C).

\begin{tabular}{|c|c|c|c|c|c|c|c|}
\hline \multirow[b]{2}{*}{ Irradiation parameter } & \multirow{2}{*}{$\begin{array}{c}\text { Reference } \\
\text { adjusted } \\
\text { value } \\
()^{a} \\
\end{array}$} & \multirow[b]{2}{*}{$\begin{array}{l}\text { Std. }^{b} \\
(\%)\end{array}$} & \multirow[b]{2}{*}{$\begin{array}{l}\text { Adj./ } \\
\text { calc. }\end{array}$} & \multirow[b]{2}{*}{$\begin{array}{l}\text { Ni58(n,p) } \\
\quad M / C\end{array}$} & \multicolumn{3}{|c|}{ Ratio $^{c}$} \\
\hline & & & & & $\begin{array}{l}\text { Added } \\
\text { Cu }\end{array}$ & $\begin{array}{c}\text { Added } \\
\text { Cu and } \\
\text { Ru }\end{array}$ & $\underset{\text { eliminated }}{\mathrm{Np}}$ \\
\hline $\begin{array}{l}\text { Neutron Flux }(E>1 \mathrm{MeV}) \\
\text { Neutron Flux }(E>0.5\end{array}$ & $2.23 \mathrm{E}+10$ & 12 & 0.80 & 0.96 & 0.95 & 1.01 & 1.03 \\
\hline $\mathrm{MeV})$ & $3.41 \mathrm{E}+10$ & 13 & 0.78 & 0.89 & 0.96 & 1.04 & 1.07 \\
\hline $\begin{array}{l}\text { Neutron Flux }(E>0.1 \mathrm{MeV}) \\
\text { Neutron Flux }(E<\end{array}$ & $5.18 \mathrm{E}+10$ & 15 & 0.75 & & 0.97 & 1.06 & 1.09 \\
\hline $0.414 \mathrm{eV})$ & $1.43 \mathrm{E}+12$ & 6 & 0.64 & & 1.00 & 1.00 & 1.00 \\
\hline $\mathrm{dpa} / \mathrm{s}(\mathrm{Fe}, \mathrm{ASTM})$ & $4.48 \mathrm{E}-11$ & 7 & 0.73 & & 0.98 & 1.03 & 1.04 \\
\hline $\mathrm{dpa} / \mathrm{s} \quad(\mathrm{Fe}, \mathrm{ENDF}-\mathrm{VI})$ & $4.35 \mathrm{E}-11$ & 7 & 0.73 & & 0.98 & 1.03 & 1.04 \\
\hline Gamma dpa/s & $6.13 \mathrm{E}-12$ & 31 & 2.22 & & 1.06 & 1.13 & 1.16 \\
\hline Gamma Flux & $2.10 \mathrm{E}+13$ & 44 & 2.23 & & 1.02 & 1.04 & 1.05 \\
\hline Neutron + Gamma dpa/s & $5.09 \mathrm{E}-11$ & 7 & 0.80 & & 0.99 & 1.04 & 1.06 \\
\hline$\chi^{2}$ per Degree of Freedom & 1.033 & & & & 1.235 & 2.067 & 1.449 \\
\hline
\end{tabular}

${ }^{\mathrm{a}}$ Units are $\mathrm{cm}^{-2} \mathrm{~s}^{-1}$ for neutron and gamma-ray fluxes and $\mathrm{s}^{-1}$ for $\mathrm{dpa} / \mathrm{s}$.

${ }^{b}$ Percent standard deviation.

${ }^{c}$ Ratio of adjusted values obtained after copper (column 6) and ruthenium measurements (column 7) were used in the adjustment, to the reference adjusted values (column 2), which were obtained without these two measurements. The column 8 provides a comparison of the adjusted values for the case in which all neptunium fission product measurements were eliminated. The reference adjustment run is given in Table 17. 
Table C.6. Comparison of measured specific activities at the two beam line 2 nozzle corner locations, 2D1C and 2D2C.

The measurements were performed during fuel cycle 400 and during fuel cycle 401 .

\begin{tabular}{|c|c|c|c|c|c|c|}
\hline & \multirow{2}{*}{ Reaction } & \multirow{2}{*}{ Cover } & \multicolumn{2}{|c|}{$\begin{array}{c}\text { Measured specific activity } \\
(\mathbf{B q} / \mathbf{m g})\end{array}$} & \multirow{2}{*}{$\begin{array}{l}\text { Ratio } \\
\text { 2D2C/2D1C }\end{array}$} & \multirow{2}{*}{$\begin{array}{l}\text { Ratio for } \\
\text { threshold } \\
\text { reactions }\end{array}$} \\
\hline & & & $\begin{array}{c}\text { 2D1C } \\
\text { (HRB2-1GC) }\end{array}$ & $\begin{array}{c}\text { 2D2C } \\
\text { (HRB2-2GC) }\end{array}$ & & \\
\hline \multirow{2}{*}{\multicolumn{7}{|c|}{$\begin{array}{l}\text { HFIR } \\
\text { Cycle } 400\end{array}$}} \\
\hline & & & & & & \\
\hline & NP237(N,F) ZR95 & GD & $2.27 \mathrm{E}+03$ & $2.64 \mathrm{E}+03$ & $\begin{array}{l}1.10 \\
1.16\end{array}$ & $\begin{array}{l}1.10 \\
1.16\end{array}$ \\
\hline & NP237(N,F) RU103 & GD & $4.56 \mathrm{E}+03$ & $2.63 \mathrm{E}+03$ & $0.58^{b}$ & \\
\hline & NP237(N,F) CS137 & GD & $1.58 \mathrm{E}+01$ & $2.10 \mathrm{E}+01$ & 1.33 & 1.33 \\
\hline & NP237(N,F) CE141 & & & $2.55 \mathrm{E}+03$ & & \\
\hline & $\operatorname{CO} 59(\mathrm{~N}, \mathrm{G}) \mathrm{CO} 60$ & GD & $2.58 \mathrm{E}+04$ & $3.56 \mathrm{E}+04$ & 1.38 & \\
\hline & $\operatorname{CO} 59(\mathrm{~N}, \mathrm{G}) \mathrm{CO} 60$ & GD & $6.14 \mathrm{E}+01^{c}$ & & & \\
\hline & $\mathrm{BE} \quad(\mathrm{N}, \mathrm{X})$ & GD & $28.8^{d}$ & $27.1^{d}$ & 0.94 & \\
\hline & & & $\begin{array}{c}\text { 2D1C } \\
\text { (HRB2-1AC) }\end{array}$ & $\begin{array}{c}\text { 2D2C } \\
\text { HRB2-2AC }\end{array}$ & Average & 1.22 \\
\hline \multicolumn{7}{|l|}{$\begin{array}{l}\text { HFIR } \\
\text { Cycle } 401\end{array}$} \\
\hline & NI58 (N,P) CO58 & $\mathrm{AL}$ & $4.32 \mathrm{E}+03$ & $5.27 \mathrm{E}+03$ & 1.22 & 1.22 \\
\hline & FE54 (N,P) MN54 & $\mathrm{AL}$ & $7.19 \mathrm{E}+01$ & $8.53 \mathrm{E}+01$ & 1.19 & 1.19 \\
\hline & CU63 (N,A) CO60 & $\mathrm{AL}$ & $1.36 \mathrm{E}+00$ & $1.70 \mathrm{E}+00$ & 1.26 & 1.26 \\
\hline & FE58 $(\mathrm{N}, \mathrm{G})$ FE59 & $\mathrm{AL}$ & $1.27 \mathrm{E}+04$ & $1.81 \mathrm{E}+04$ & 1.42 & \\
\hline & $\operatorname{CO} 59(\mathrm{~N}, \mathrm{G}) \mathrm{CO} 60$ & $\mathrm{AL}$ & $3.46 \mathrm{E}+06$ & $4.03 \mathrm{E}+06$ & 1.16 & \\
\hline & $\operatorname{CO} 59(\mathrm{~N}, \mathrm{G}) \mathrm{CO} 60$ & $\mathrm{AL}$ & $3.73 \mathrm{E}+03^{\mathrm{c}}$ & & & \\
\hline & & & & & Average & 1.22 \\
\hline
\end{tabular}

\footnotetext{
${ }^{a}$ The threshold reactions only are compared here because they represent better the energetic part of the neutron spectrum, which is responsible for dpa rate.

${ }^{b}$ The measured activity of Ru-103 was ignored in the comparison because it is largely inconsistent with other activities.

${ }^{c}$ Diluted cobalt in form of Co-Al alloy.

${ }^{d}$ Concentration of helium in beryllium sample is in atom parts per billion (appb; $10 \mathrm{E}-9$ atom fraction).
} 
Table C.7. Comparison of calculated and "measured" reaction rates for the Key 2, positions 2D1C and 2D2C

\begin{tabular}{|c|c|c|c|c|c|}
\hline \multirow[b]{2}{*}{ Location } & \multirow[b]{2}{*}{ Reaction } & \multirow[b]{2}{*}{ Cover } & \multicolumn{2}{|c|}{ Reaction rate } & \multirow[b]{2}{*}{$\mathbf{M} / \mathbf{C}$} \\
\hline & & & $\begin{array}{c}\text { Calculated } \\
\left(\mathrm{s}^{-1}\right)\end{array}$ & $\begin{array}{c}\text { Measured } \\
\left(\mathrm{s}^{-1}\right)\end{array}$ & \\
\hline \multirow[t]{8}{*}{ Key 2, 2D2C } & NI58 (N,P) CO58 & GD & $3.24 \mathrm{E}-15$ & $3.59 \mathrm{E}-15$ & 1.11 \\
\hline & NI58 (N,P) CO58 & & $3.29 \mathrm{E}-15$ & $3.56 \mathrm{E}-15$ & 1.08 \\
\hline & $\mathrm{BE} \quad(\mathrm{N}, \mathrm{X})$ & GD & $1.08 \mathrm{E}-14$ & $1.29 \mathrm{E}-14$ & 1.19 \\
\hline & NP237(N,F) ZR95 & GD & $7.89 \mathrm{E}-14$ & $7.92 \mathrm{E}-14$ & 1.00 \\
\hline & NP237(N,F) CS137 & GD & $7.89 \mathrm{E}-14$ & 8.77E-14 & 1.11 \\
\hline & FE54 (N,P) MN54 & & $2.45 \mathrm{E}-15$ & $2.55 \mathrm{E}-15$ & 1.04 \\
\hline & FE58 (N,G) FE59 & & $2.05 \mathrm{E}-12$ & $1.90 \mathrm{E}-12$ & 0.93 \\
\hline & $\operatorname{CO} 59(\mathrm{~N}, \mathrm{G}) \mathrm{CO} 60$ & & $6.67 \mathrm{E}-11$ & $6.10 \mathrm{E}-11$ & 0.91 \\
\hline \multirow[t]{9}{*}{ Key 2, 2D1C } & NI58 (N,P) CO58 & GD & $3.24 \mathrm{E}-15$ & $3.10 \mathrm{E}-15$ & 0.96 \\
\hline & NI58 (N,P) CO58 & & $3.29 \mathrm{E}-15$ & $2.92 \mathrm{E}-15$ & 0.89 \\
\hline & $\mathrm{BE} \quad(\mathrm{N}, \mathrm{X})$ & GD & $1.08 \mathrm{E}-14$ & $1.37 \mathrm{E}-14$ & 1.27 \\
\hline & NP237(N,F) ZR95 & GD & $7.89 \mathrm{E}-14$ & $6.81 \mathrm{E}-14$ & 0.86 \\
\hline & NP237(N,F) CS137 & GD & $7.89 \mathrm{E}-14$ & $6.58 \mathrm{E}-14$ & 0.83 \\
\hline & FE54 (N,P) MN54 & & $2.45 \mathrm{E}-15$ & $2.15 \mathrm{E}-15$ & 0.88 \\
\hline & FE58 (N,G) FE59 & & $2.05 \mathrm{E}-12$ & $1.33 \mathrm{E}-12$ & 0.65 \\
\hline & $\operatorname{CO} 59(\mathrm{~N}, \mathrm{G}) \mathrm{CO} 60$ & & $6.67 \mathrm{E}-11$ & $4.18 \mathrm{E}-11$ & 0.63 \\
\hline & $\operatorname{CO} 59(\mathrm{~N}, \mathrm{G}) \mathrm{CO} 60$ & GD & $1.57 \mathrm{E}-12$ & $6.92 \mathrm{E}-13$ & 0.44 \\
\hline
\end{tabular}




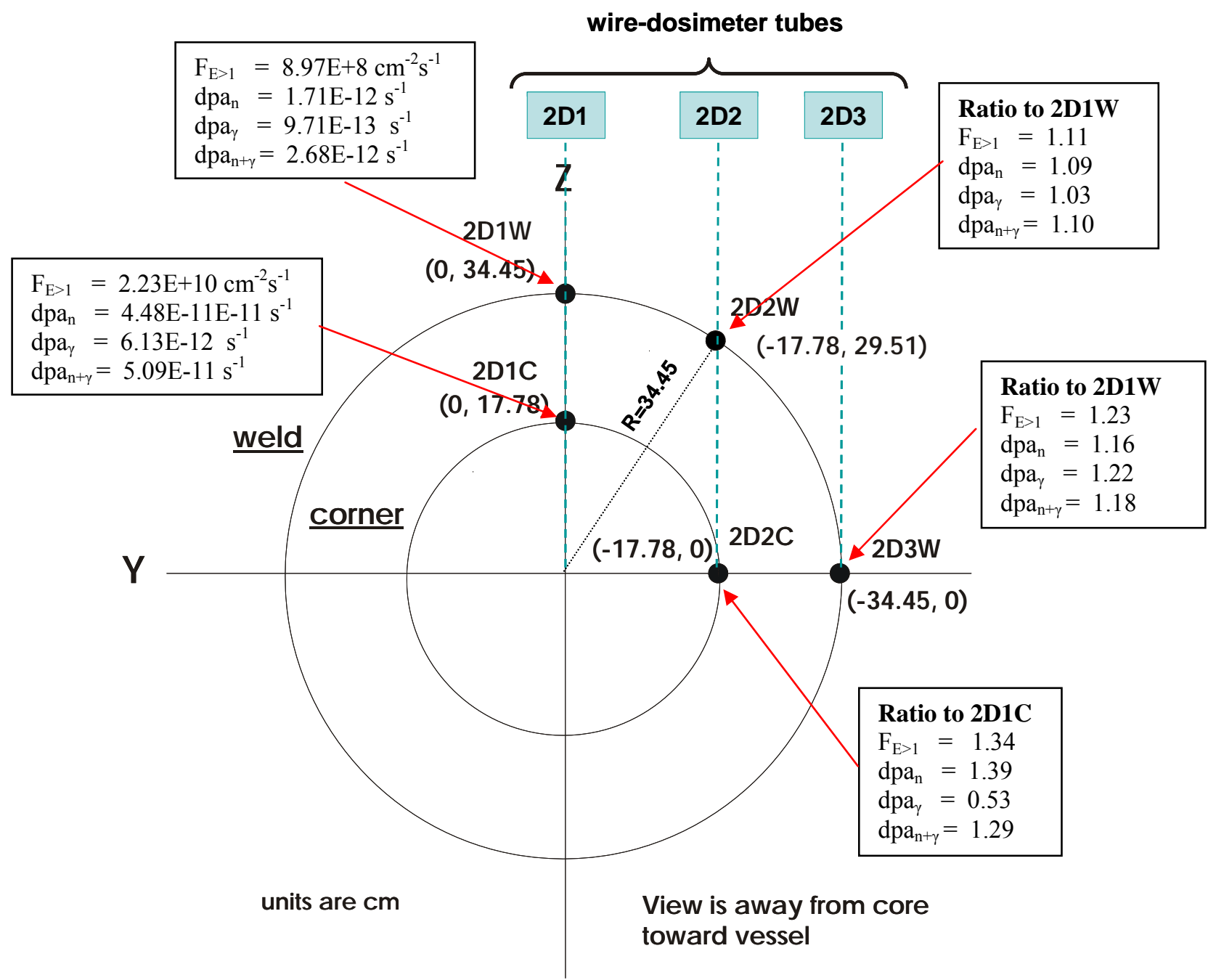

Fig. C.1. Comparison of the adjusted irradiation parameters at the dosimeter locations inside the tubular dosimeters sets located at Key 2. 
Table C.8. Calculated (obtained from transport calculations) and modified ("new") flux values for the top five energy groups, for the location 2D1C

\begin{tabular}{|c|c|c|c|c|c|}
\hline \multirow[b]{2}{*}{ Group } & \multicolumn{2}{|c|}{ Group boundaries } & \multicolumn{2}{|c|}{$\begin{array}{c}\text { Neutron flux } \\
\end{array}$} & \multirow[b]{2}{*}{ Ratio } \\
\hline & $\begin{array}{l}\text { Top } \\
(\mathrm{eV})\end{array}$ & $\begin{array}{c}\text { Bottom } \\
(\mathrm{eV})\end{array}$ & $\begin{array}{c}\text { Calculated } \\
\left(\mathrm{cm}^{-2} \mathrm{~s}^{-1}\right)\end{array}$ & $\begin{array}{c}\mathrm{New} \\
\left(\mathrm{cm}^{-2} \mathrm{~s}^{-1}\right)\end{array}$ & \\
\hline 1 & $1.49 \mathrm{E}+07$ & $1.22 \mathrm{E}+07$ & $1.36 \mathrm{E}+07$ & $2.20 \mathrm{E}+07$ & 1.62 \\
\hline 2 & $1.22 \mathrm{E}+07$ & $1.00 \mathrm{E}+07$ & $5.46 \mathrm{E}+07$ & $8.38 \mathrm{E}+07$ & 1.54 \\
\hline 3 & $1.00 \mathrm{E}+07$ & $8.19 \mathrm{E}+06$ & $1.56 \mathrm{E}+08$ & $2.32 \mathrm{E}+08$ & 1.49 \\
\hline 4 & $8.19 \mathrm{E}+06$ & $6.70 \mathrm{E}+06$ & $3.80 \mathrm{E}+08$ & $4.52 \mathrm{E}+08$ & 1.19 \\
\hline 5 & $6.70 \mathrm{E}+06$ & $5.49 \mathrm{E}+06$ & $7.73 \mathrm{E}+08$ & $8.32 \mathrm{E}+08$ & 1.08 \\
\hline
\end{tabular}

Table C.9. Comparison of two adjustment runs for location 2D1C

\begin{tabular}{|c|c|c|c|c|c|}
\hline Irradiation parameter & $\begin{array}{c}\text { “Reference” values } \\
\text { (from Table 17) } \\
\text { ( ) }\end{array}$ & $\begin{array}{l}\text { Std. }^{b} \\
(\%)\end{array}$ & $\begin{array}{c}\text { New } \\
\text { adjusted } \\
\text { value } \\
()^{a}\end{array}$ & $\begin{array}{l}\text { Std. }{ }^{c} \\
(\%)\end{array}$ & $\begin{array}{c}\text { Reference/ } \\
\text { new }\end{array}$ \\
\hline Neutron flux $(\mathrm{E}>1 \mathrm{MeV})$ & $2.23 \mathrm{E}+10$ & 11.6 & $2.14 \mathrm{E}+10$ & 11.2 & 1.04 \\
\hline Neutron flux $(E>0.5 \mathrm{MeV})$ & $3.41 \mathrm{E}+10$ & 12.7 & $3.29 \mathrm{E}+10$ & 12.5 & 1.04 \\
\hline Neutron flux $(\mathrm{E}>0.1 \mathrm{MeV})$ & $5.18 \mathrm{E}+10$ & 15.3 & $5.02 \mathrm{E}+10$ & 15.2 & 1.03 \\
\hline Neutron flux $(\mathrm{E}<0.414 \mathrm{eV})$ & $1.43 \mathrm{E}+12$ & 6.1 & $1.43 \mathrm{E}+12$ & 6.1 & 1.00 \\
\hline $\mathrm{dpa} / \mathrm{s}(\mathrm{Fe}, \mathrm{ASTM})$ & $4.48 \mathrm{E}-11$ & 7.1 & $4.39 \mathrm{E}-11$ & 7 & 1.02 \\
\hline $\mathrm{dpa} / \mathrm{s}(\mathrm{Fe}, \mathrm{ENDF}-\mathrm{VI})$ & $4.35 \mathrm{E}-11$ & 7.4 & $4.27 \mathrm{E}-11$ & 7.3 & 1.02 \\
\hline Gamma dpa/s & $6.13 \mathrm{E}-12$ & 30.7 & $6.59 \mathrm{E}-12$ & 31.2 & 0.93 \\
\hline Gamma flux & $2.10 \mathrm{E}+13$ & 43.6 & $2.19 \mathrm{E}+13$ & 44 & 0.96 \\
\hline Neutron + gamma dpa/s & $5.09 \mathrm{E}-11$ & 7 & $5.05 \mathrm{E}-11$ & 7 & 1.01 \\
\hline
\end{tabular}




\section{APPENDIX D}

ADJUSTED IRRADIATION PARAMETERS AS GIVEN IN THE PRELIMINARY ANALYSIS REPORT, DATED NOVEMBER 18, 2005 

In November 2005, the preliminary results from the analysis of the dosimetry experiments performed during HFIR fuel cycles 400 and 401 were made available in order to make possible the timely safety analysis of the HFIR pressure vessel. The informal report, "Analysis of HFIR Dosimetry Experiments Performed in Cycles 400 and 401," was prepared by I. Remec and C. Baldwin and submitted as a personal communication in an e-mail to J. Inger and D. Cheverton.

In the preliminary analysis, the calculated neutron and gamma-ray fluxes for one location only within each dosimetry capsule were used in the adjustment calculations. The adjustment calculations were done in such a way that the calculated fluxes were first normalized to the measured NI58 (N,P) CO58 reaction at the position of each dosimetry vial in the capsule, and then the adjustment was performed. Such a procedure is recommended when variations in the magnitude of the flux from one location to another are present; however, little variation in the energy distribution of the flux (flux spectrum) is expected. While such a procedure was considered appropriate, it was nevertheless suggested that we obtain the calculated fluxes from the transport calculations at each vial location for capsules where significant gradients were observed and repeat the adjustment calculations. This was, in fact, performed for this final report. The dosimetry capsules with significant gradients were DOS-1, DOS-2, DOS-3, DOS-4, and DOS-5. For these capsules the adjustment calculations were repeated. The adjusted parameters for these locations from the preliminary report are provided in Tables D. 1 to D.6 along with corresponding values from the final adjustment runs. In Tables D.2 to D.6, the comparison of the final adjusted irradiation parameters to those from the preliminary report are provided in the column labeled "Adjusted New/Old." For all other dosimeter capsules, the adjusted values in the final and preliminary analysis are the same and are not repeated here.

At the center of the dosimetry capsules, the irradiation parameters from the preliminary and the final analysis are in most cases identical, with occasional small changes that do not exceed 3\%. This central location corresponds to the V-notch of the actual Charpy samples and is the only location of importance for evaluation of Charpy test results.

At Position 1 and Position 3, which are closer to the "top" and "bottom' of the capsule, respectively, the differences in the irradiation parameters from the preliminary and the final analysis are typically on the order of a few percent (smaller than $\sim 5 \%$ ) and reach a maximum of 13\% in one case (in capsule DOS-4).

The irradiation parameter values provided in the preliminary report are therefore consistent with the values from this final report. 
Table D.1. Summary of the adjusted irradiation parameters from the preliminary analysis

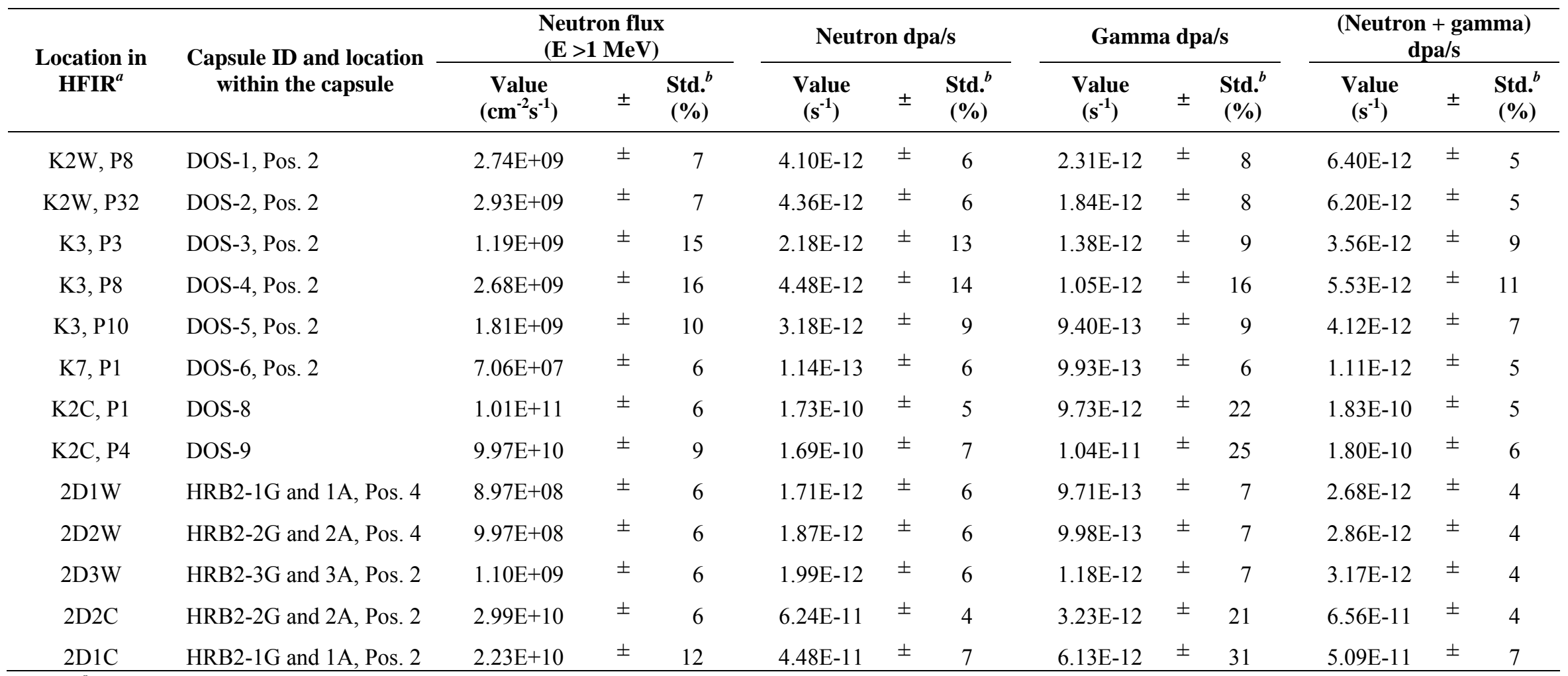

${ }^{a} \mathrm{~K}=$ key, $\mathrm{P}=$ position in key, $\mathrm{W}=$ nozzle weld, $\mathrm{C}=$ nozzle corner, $2 \mathrm{D}=$ tubular dosimeter at HB-2.

${ }^{b}$ Percent standard deviation. 
Table D.2. Calculated (for Position 2) and adjusted irradiation parameters for capsule DOS-1, at $\mathrm{K} 2 \mathrm{~W}, \mathrm{P8}$ from the preliminary analysis and comparison with the final analysis

\begin{tabular}{|c|c|c|c|c|c|c|c|c|}
\hline & Irradiation parameter & $\begin{array}{c}\text { Calculated } \\
\text { value } \\
()^{a}\end{array}$ & $\begin{array}{l}\text { Std. }^{b} \\
(\%)\end{array}$ & $\begin{array}{c}\text { Adjusted } \\
\text { value } \\
()^{a}\end{array}$ & $\begin{array}{l}\text { Std. }^{b} \\
(\%)\end{array}$ & $\begin{array}{l}\text { Adj./ } \\
\text { calc. }\end{array}$ & $\begin{array}{c}\text { Ni58(n,p) } \\
\text { M/C }\end{array}$ & $\begin{array}{c}\text { Adjusted } \\
\text { new/old }^{c}\end{array}$ \\
\hline \multirow{6}{*}{ Position 1} & Neutron flux $(\mathrm{E}>1 \mathrm{MeV})$ & $2.36 \mathrm{E}+09$ & 19 & $2.10 \mathrm{E}+09$ & 7 & 0.89 & \multirow[t]{6}{*}{0.91} & 0.98 \\
\hline & Neutron flux $(\mathrm{E}>0.5 \mathrm{MeV})$ & $3.52 \mathrm{E}+09$ & 19 & $3.12 \mathrm{E}+09$ & 7 & 0.89 & & 0.97 \\
\hline & Neutron flux $(\mathrm{E}>0.1 \mathrm{MeV})$ & $5.07 \mathrm{E}+09$ & 19 & $4.46 \mathrm{E}+09$ & 8 & 0.88 & & 0.96 \\
\hline & Neutron flux $(\mathrm{E}<0.414 \mathrm{eV})$ & $1.69 \mathrm{E}+10$ & 40 & $1.15 \mathrm{E}+10$ & 6 & 0.68 & & 1.00 \\
\hline & $\mathrm{dpa} / \mathrm{s}(\mathrm{Fe}, \mathrm{ASTM})$ & $3.57 \mathrm{E}-12$ & 18 & $3.13 \mathrm{E}-12$ & 6 & 0.88 & & 0.98 \\
\hline & $\mathrm{dpa} / \mathrm{s}(\mathrm{Fe}, \mathrm{ENDF}-\mathrm{VI})$ & $3.53 \mathrm{E}-12$ & 18 & $3.09 \mathrm{E}-12$ & 6 & 0.88 & & 0.98 \\
\hline \multirow{9}{*}{ Position 2} & Neutron flux $(\mathrm{E}>1 \mathrm{MeV})$ & $2.36 \mathrm{E}+09$ & 19 & $2.74 \mathrm{E}+09$ & 7 & 1.16 & \multirow[t]{9}{*}{1.20} & 1.00 \\
\hline & Neutron flux $(\mathrm{E}>0.5 \mathrm{MeV})$ & $3.52 \mathrm{E}+09$ & 19 & $4.07 \mathrm{E}+09$ & 8 & 1.16 & & 1.00 \\
\hline & Neutron flux $(\mathrm{E}>0.1 \mathrm{MeV})$ & $5.07 \mathrm{E}+09$ & 19 & $5.82 \mathrm{E}+09$ & 8 & 1.15 & & 1.00 \\
\hline & Neutron flux $(\mathrm{E}<0.414 \mathrm{eV})$ & $1.69 \mathrm{E}+10$ & 40 & $1.74 \mathrm{E}+10$ & 22 & 1.03 & & 0.98 \\
\hline & $\mathrm{dpa} / \mathrm{s}$ (Fe, ASTM) & $3.57 \mathrm{E}-12$ & 18 & $4.10 \mathrm{E}-12$ & 6 & 1.15 & & 1.00 \\
\hline & $\mathrm{dpa} / \mathrm{s}(\mathrm{Fe}, \mathrm{ENDF}-\mathrm{VI})$ & $3.53 \mathrm{E}-12$ & 18 & $4.05 \mathrm{E}-12$ & 6 & 1.15 & & 1.00 \\
\hline & Gamma dpa/s & $2.00 \mathrm{E}-12$ & 27 & $2.31 \mathrm{E}-12$ & 8 & 1.15 & & 1.00 \\
\hline & Gamma flux & $8.17 \mathrm{E}+12$ & 28 & $9.85 \mathrm{E}+12$ & 16 & 1.21 & & 1.00 \\
\hline & Neutron + gamma dpa/s & $5.57 \mathrm{E}-12$ & & $6.40 \mathrm{E}-12$ & 5 & 1.15 & & 1.00 \\
\hline \multirow{6}{*}{ Position 3} & Neutron flux $(\mathrm{E}>1 \mathrm{MeV})$ & $2.36 \mathrm{E}+09$ & 19 & $3.71 \mathrm{E}+09$ & 7 & 1.57 & \multirow[t]{6}{*}{1.60} & 1.03 \\
\hline & Neutron flux $(\mathrm{E}>0.5 \mathrm{MeV})$ & $3.52 \mathrm{E}+09$ & 19 & $5.53 \mathrm{E}+09$ & 7 & 1.57 & & 1.05 \\
\hline & Neutron flux $(\mathrm{E}>0.1 \mathrm{MeV})$ & $5.07 \mathrm{E}+09$ & 19 & $7.93 \mathrm{E}+09$ & 8 & 1.57 & & 1.06 \\
\hline & Neutron flux $(\mathrm{E}<0.414 \mathrm{eV})$ & $1.69 \mathrm{E}+10$ & 40 & $3.35 \mathrm{E}+10$ & 6 & 1.98 & & 1.00 \\
\hline & $\mathrm{dpa} / \mathrm{s}(\mathrm{Fe}, \mathrm{ASTM})$ & $3.57 \mathrm{E}-12$ & 18 & $5.65 \mathrm{E}-12$ & 6 & 1.58 & & 1.04 \\
\hline & $\mathrm{dpa} / \mathrm{s}(\mathrm{Fe}, \mathrm{ENDF}-\mathrm{VI})$ & $3.53 \mathrm{E}-12$ & 18 & $5.58 \mathrm{E}-12$ & 6 & 1.58 & & 1.04 \\
\hline
\end{tabular}

${ }^{a}$ Units are $\mathrm{cm}^{-2} \mathrm{~s}^{-1}$ for neutron and gamma-ray fluxes and $\mathrm{s}^{-1}$ for $\mathrm{dpa} / \mathrm{s}$.

${ }^{b}$ Percent standard deviation. The $\chi 2$ per degree of freedom for the adjustment run was $1.024 \mathrm{E}+00$.

"New" adjustment was done with calculated fluxes for each of the three positions; "old" adjustment was done with calculated fluxes for Position 2 only. 
Table D.3. Calculated (for Position 2 only) and adjusted irradiation parameters for capsule DOS-2, at K2W, P3 from the preliminary analysis and comparison with the final analysis

\begin{tabular}{|c|c|c|c|c|c|c|c|c|}
\hline & Irradiation parameter & $\begin{array}{c}\text { Calculated } \\
\text { value } \\
()^{a}\end{array}$ & $\begin{array}{l}\text { Std. }^{b} \\
(\%)\end{array}$ & $\begin{array}{c}\text { Adjusted } \\
\text { value } \\
()^{a}\end{array}$ & $\begin{array}{l}\text { Std. }^{b} \\
(\%)\end{array}$ & $\begin{array}{l}\text { Adj./ } \\
\text { calc. }\end{array}$ & $\begin{array}{c}\text { Ni58(n,p) } \\
\text { M/C }\end{array}$ & $\begin{array}{l}\text { Adjusted } \\
\text { new/old }^{c}\end{array}$ \\
\hline \multirow{6}{*}{ Position 1} & Neutron flux $(\mathrm{E}>1 \mathrm{MeV})$ & $2.36 \mathrm{E}+09$ & 19 & $2.26 \mathrm{E}+09$ & 7 & 0.96 & \multirow[t]{6}{*}{0.97} & 0.98 \\
\hline & Neutron flux $(E>0.5 \mathrm{MeV})$ & $3.52 \mathrm{E}+09$ & 19 & $3.37 \mathrm{E}+09$ & 7 & 0.96 & & 0.97 \\
\hline & Neutron flux $(\mathrm{E}>0.1 \mathrm{MeV})$ & $5.07 \mathrm{E}+09$ & 19 & $4.80 \mathrm{E}+09$ & 8 & 0.95 & & 0.96 \\
\hline & Neutron flux $(\mathrm{E}<0.414 \mathrm{eV})$ & $1.69 \mathrm{E}+10$ & 40 & $9.77 \mathrm{E}+09$ & 5 & 0.58 & & 1.00 \\
\hline & $\mathrm{dpa} / \mathrm{s}(\mathrm{Fe}, \mathrm{ASTM})$ & $3.57 \mathrm{E}-12$ & 18 & $3.35 \mathrm{E}-12$ & 6 & 0.94 & & 0.98 \\
\hline & $\mathrm{dpa} / \mathrm{s} \quad(\mathrm{Fe}, \mathrm{ENDF}-\mathrm{VI})$ & $3.53 \mathrm{E}-12$ & 18 & $3.31 \mathrm{E}-12$ & 6 & 0.94 & & 0.98 \\
\hline \multirow{9}{*}{ Position 2} & Neutron flux $(\mathrm{E}>1 \mathrm{MeV})$ & $2.36 \mathrm{E}+09$ & 19 & $2.93 \mathrm{E}+09$ & 7 & 1.24 & \multirow[t]{9}{*}{1.29} & 1.00 \\
\hline & Neutron flux $(\mathrm{E}>0.5 \mathrm{MeV})$ & $3.52 \mathrm{E}+09$ & 19 & $4.36 \mathrm{E}+09$ & 8 & 1.24 & & 1.00 \\
\hline & Neutron flux $(\mathrm{E}>0.1 \mathrm{MeV})$ & $5.07 \mathrm{E}+09$ & 19 & $6.22 \mathrm{E}+09$ & 8 & 1.23 & & 1.00 \\
\hline & Neutron flux $(\mathrm{E}<0.414 \mathrm{eV})$ & $1.69 \mathrm{E}+10$ & 40 & $1.49 \mathrm{E}+10$ & 22 & 0.88 & & 0.98 \\
\hline & $\mathrm{dpa} / \mathrm{s}(\mathrm{Fe}, \mathrm{ASTM})$ & $3.57 \mathrm{E}-12$ & 18 & $4.36 \mathrm{E}-12$ & 6 & 1.22 & & 1.00 \\
\hline & $\mathrm{dpa} / \mathrm{s} \quad(\mathrm{Fe}, \mathrm{ENDF}-\mathrm{VI})$ & $3.53 \mathrm{E}-12$ & 18 & $4.31 \mathrm{E}-12$ & 6 & 1.22 & & 1.00 \\
\hline & Gamma dpa/s & $2.00 \mathrm{E}-12$ & 27 & $1.84 \mathrm{E}-12$ & 8 & 0.92 & & 1.00 \\
\hline & Gamma flux & $8.17 \mathrm{E}+12$ & 28 & $7.86 \mathrm{E}+12$ & 16 & 0.96 & & 1.00 \\
\hline & Neutron + gamma dpa/s & $5.57 \mathrm{E}-12$ & & $6.20 \mathrm{E}-12$ & 5 & 1.11 & & 1.00 \\
\hline \multirow{6}{*}{ Position 3} & Neutron flux $(\mathrm{E}>1 \mathrm{MeV})$ & $2.36 \mathrm{E}+09$ & 19 & $3.86 \mathrm{E}+09$ & 7 & 1.64 & \multirow[t]{6}{*}{1.70} & 1.03 \\
\hline & Neutron flux $(E>0.5 \mathrm{MeV})$ & $3.52 \mathrm{E}+09$ & 19 & $5.76 \mathrm{E}+09$ & 7 & 1.64 & & 1.05 \\
\hline & Neutron flux $(\mathrm{E}>0.1 \mathrm{MeV})$ & $5.07 \mathrm{E}+09$ & 19 & $8.24 \mathrm{E}+09$ & 8 & 1.63 & & 1.06 \\
\hline & Neutron flux $(\mathrm{E}<0.414 \mathrm{eV})$ & $1.69 \mathrm{E}+10$ & 40 & $2.54 \mathrm{E}+10$ & 5 & 1.50 & & 1.00 \\
\hline & $\mathrm{dpa} / \mathrm{s}(\mathrm{Fe}, \mathrm{ASTM})$ & $3.57 \mathrm{E}-12$ & 18 & $5.80 \mathrm{E}-12$ & 6 & 1.63 & & 1.04 \\
\hline & $\mathrm{dpa} / \mathrm{s} \quad(\mathrm{Fe}, \mathrm{ENDF}-\mathrm{VI})$ & $3.53 \mathrm{E}-12$ & 18 & $5.74 \mathrm{E}-12$ & 6 & 1.63 & & 1.04 \\
\hline
\end{tabular}

${ }^{a}$ Units are $\mathrm{cm}^{-2} \mathrm{~s}^{-1}$ for neutron and gamma-ray fluxes and $\mathrm{s}^{-1}$ for $\mathrm{dpa} / \mathrm{s}$.

${ }^{b}$ Percent standard deviation. The $\chi 2$ per degree of freedom for the adjustment run was $8.536 \mathrm{E}-01$.

"New" adjustment was done with calculated fluxes for each of the three positions; "old" adjustment was done with calculated fluxes for Position 2 only. 
Table D.4. Calculated (for Position 2 only) and adjusted irradiation parameters for capsule DOS-3, at K3, P3 from the preliminary analysis and comparison with the final analysis

\begin{tabular}{|c|c|c|c|c|c|c|c|c|}
\hline & Irradiation parameter & $\begin{array}{c}\text { Calculated } \\
\text { value } \\
()^{a}\end{array}$ & $\begin{array}{l}\text { Std. }^{b} \\
(\%)\end{array}$ & $\begin{array}{c}\text { Adjusted } \\
\text { value } \\
()^{a}\end{array}$ & $\begin{array}{l}\text { Std. }^{b} \\
(\%)\end{array}$ & $\begin{array}{l}\text { Adj./ } \\
\text { calc. }\end{array}$ & $\begin{array}{c}\text { Ni58(n,p) } \\
\text { M/C }\end{array}$ & $\begin{array}{l}\text { Adjusted } \\
\text { new/old }^{c}\end{array}$ \\
\hline \multirow{6}{*}{ Position 1} & Neutron Flux (E > 1MeV) & $1.32 \mathrm{E}+09$ & 58 & $7.63 \mathrm{E}+08$ & 15 & 0.58 & \multirow[t]{6}{*}{0.62} & 0.96 \\
\hline & Neutron Flux $(\mathrm{E}>0.5 \mathrm{MeV})$ & $2.27 \mathrm{E}+09$ & 58 & $1.33 \mathrm{E}+09$ & 18 & 0.59 & & 0.96 \\
\hline & Neutron Flux $(\mathrm{E}>0.1 \mathrm{MeV})$ & $3.75 \mathrm{E}+09$ & 57 & $2.18 \mathrm{E}+09$ & 20 & 0.58 & & 0.96 \\
\hline & Neutron Flux $(\mathrm{E}<0.414 \mathrm{eV})$ & $3.07 \mathrm{E}+10$ & 60 & $1.36 \mathrm{E}+10$ & 7 & 0.44 & & 0.99 \\
\hline & $\mathrm{dpa} / \mathrm{s}(\mathrm{Fe}, \mathrm{ASTM})$ & $2.31 \mathrm{E}-12$ & 50 & $1.31 \mathrm{E}-12$ & 13 & 0.56 & & 0.97 \\
\hline & $\mathrm{dpa} / \mathrm{s} \quad(\mathrm{Fe}, \mathrm{ENDF}-\mathrm{VI})$ & $2.29 \mathrm{E}-12$ & 51 & $1.30 \mathrm{E}-12$ & 14 & 0.57 & & 0.97 \\
\hline \multirow{9}{*}{ Position 2} & Neutron Flux (E > 1MeV) & $1.32 \mathrm{E}+09$ & 58 & $1.19 \mathrm{E}+09$ & 15 & 0.91 & \multirow[t]{9}{*}{0.95} & 0.98 \\
\hline & Neutron Flux $(\mathrm{E}>0.5 \mathrm{MeV})$ & $2.27 \mathrm{E}+09$ & 58 & $2.08 \mathrm{E}+09$ & 17 & 0.92 & & 0.98 \\
\hline & Neutron Flux $(\mathrm{E}>0.1 \mathrm{MeV})$ & $3.75 \mathrm{E}+09$ & 57 & $3.46 \mathrm{E}+09$ & 20 & 0.92 & & 0.98 \\
\hline & Neutron Flux $(\mathrm{E}<0.414 \mathrm{eV})$ & $3.07 \mathrm{E}+10$ & 60 & $3.48 \mathrm{E}+10$ & 38 & 1.13 & & 0.97 \\
\hline & $\mathrm{dpa} / \mathrm{s}(\mathrm{Fe}, \mathrm{ASTM})$ & $2.31 \mathrm{E}-12$ & 50 & $2.18 \mathrm{E}-12$ & 13 & 0.94 & & 0.99 \\
\hline & dpa/s (Fe, ENDF-VI) & $2.29 \mathrm{E}-12$ & 51 & $2.16 \mathrm{E}-12$ & 13 & 0.94 & & 0.99 \\
\hline & Gamma dpa/s & $9.84 \mathrm{E}-13$ & 54 & $1.38 \mathrm{E}-12$ & 9 & 1.40 & & 1.00 \\
\hline & Gamma Flux & $3.25 \mathrm{E}+12$ & 56 & $4.72 \mathrm{E}+12$ & 28 & 1.45 & & 0.99 \\
\hline & Neutron + Gamma dpa/s & $3.30 \mathrm{E}-12$ & & $3.56 \mathrm{E}-12$ & 9 & 1.08 & & 0.99 \\
\hline \multirow{6}{*}{ Position 3} & Neutron Flux $(\mathrm{E}>1 \mathrm{MeV})$ & $1.32 \mathrm{E}+09$ & 58 & $1.81 \mathrm{E}+09$ & 15 & 1.38 & \multirow[t]{6}{*}{1.44} & 1.01 \\
\hline & Neutron Flux $(\mathrm{E}>0.5 \mathrm{MeV})$ & $2.27 \mathrm{E}+09$ & 58 & $3.20 \mathrm{E}+09$ & 18 & 1.41 & & 1.01 \\
\hline & Neutron Flux $(\mathrm{E}>0.1 \mathrm{MeV})$ & $3.75 \mathrm{E}+09$ & 57 & $5.43 \mathrm{E}+09$ & 20 & 1.45 & & 1.00 \\
\hline & Neutron Flux $(\mathrm{E}<0.414 \mathrm{eV})$ & $3.07 \mathrm{E}+10$ & 60 & $1.03 \mathrm{E}+11$ & 7 & 3.37 & & 1.01 \\
\hline & $\mathrm{dpa} / \mathrm{s}(\mathrm{Fe}, \mathrm{ASTM})$ & $2.31 \mathrm{E}-12$ & 50 & $3.88 \mathrm{E}-12$ & 13 & 1.68 & & 1.00 \\
\hline & $\mathrm{dpa} / \mathrm{s}$ (Fe, ENDF-VI) & $2.29 \mathrm{E}-12$ & 51 & $3.81 \mathrm{E}-12$ & 14 & 1.66 & & 1.00 \\
\hline
\end{tabular}

${ }^{a}$ Units are $\mathrm{cm}^{-2} \mathrm{~s}^{-1}$ for neutron and gamma-ray fluxes and $\mathrm{s}^{-1}$ for $\mathrm{dpa} / \mathrm{s}$.

${ }^{b}$ Percent standard deviation. The $\chi 2$ per degree of freedom for the adjustment run was $9.865 \mathrm{E}-01$.

"New" adjustment was done with calculated fluxes for each of the three positions; "old" adjustment was done with calculated fluxes for Position 2 only. 
Table D.5. Calculated (for Position 2 only) and adjusted irradiation parameters for capsule DOS-4, at K3, P8 from the preliminary analysis and comparison with the final analysis

\begin{tabular}{|c|c|c|c|c|c|c|c|c|}
\hline & Irradiation parameter & $\begin{array}{c}\text { Calculated } \\
\text { value } \\
()^{a}\end{array}$ & $\begin{array}{l}\text { Std. }^{b} \\
(\%)\end{array}$ & $\begin{array}{c}\text { Adjusted } \\
\text { value } \\
()^{a}\end{array}$ & $\begin{array}{l}\text { Std. }^{b} \\
(\%)\end{array}$ & $\begin{array}{l}\text { Adj./ } \\
\text { calc. }\end{array}$ & $\begin{array}{c}\text { Ni58(n,p) } \\
\text { M/C }\end{array}$ & $\begin{array}{l}\text { Adjusted } \\
\text { new/old }^{c}\end{array}$ \\
\hline \multirow{6}{*}{ Position 1} & Neutron flux (E > 1MeV) & $2.32 \mathrm{E}+09$ & 53 & $2.05 \mathrm{E}+09$ & 16 & 0.88 & \multirow[t]{6}{*}{0.89} & 0.86 \\
\hline & Neutron flux $(E>0.5 \mathrm{MeV})$ & $3.91 \mathrm{E}+09$ & 53 & $3.43 \mathrm{E}+09$ & 18 & 0.88 & & 0.83 \\
\hline & Neutron flux $(\mathrm{E}>0.1 \mathrm{MeV})$ & $6.39 \mathrm{E}+09$ & 52 & $5.49 \mathrm{E}+09$ & 21 & 0.86 & & 0.82 \\
\hline & Neutron flux $(\mathrm{E}<0.414 \mathrm{eV})$ & $4.27 \mathrm{E}+10$ & 55 & $1.93 \mathrm{E}+10$ & 7 & 0.45 & & 1.00 \\
\hline & $\mathrm{dpa} / \mathrm{s}(\mathrm{Fe}, \mathrm{ASTM})$ & $3.99 \mathrm{E}-12$ & 47 & $3.28 \mathrm{E}-12$ & 13 & 0.82 & & 0.87 \\
\hline & $\mathrm{dpa} / \mathrm{s}(\mathrm{Fe}, \mathrm{ENDF}-\mathrm{VI})$ & $3.96 \mathrm{E}-12$ & 47 & $3.26 \mathrm{E}-12$ & 14 & 0.82 & & 0.87 \\
\hline \multirow{9}{*}{ Position 2} & Neutron flux $(\mathrm{E}>1 \mathrm{MeV})$ & $2.32 \mathrm{E}+09$ & 53 & $2.68 \mathrm{E}+09$ & 16 & 1.16 & \multirow[t]{9}{*}{1.16} & 0.98 \\
\hline & Neutron flux $(\mathrm{E}>0.5 \mathrm{MeV})$ & $3.91 \mathrm{E}+09$ & 53 & $4.52 \mathrm{E}+09$ & 18 & 1.16 & & 0.98 \\
\hline & Neutron flux $(\mathrm{E}>0.1 \mathrm{MeV})$ & $6.39 \mathrm{E}+09$ & 52 & $7.30 \mathrm{E}+09$ & 20 & 1.14 & & 0.99 \\
\hline & Neutron flux $(\mathrm{E}<0.414 \mathrm{eV})$ & $4.27 \mathrm{E}+10$ & 55 & $4.11 \mathrm{E}+10$ & 35 & 0.96 & & 0.88 \\
\hline & $\mathrm{dpa} / \mathrm{s}(\mathrm{Fe}, \mathrm{ASTM})$ & $3.99 \mathrm{E}-12$ & 47 & $4.48 \mathrm{E}-12$ & 14 & 1.12 & & 0.98 \\
\hline & $\mathrm{dpa} / \mathrm{s}$ (Fe, ENDF-VI) & $3.96 \mathrm{E}-12$ & 47 & $4.45 \mathrm{E}-12$ & 14 & 1.12 & & 0.98 \\
\hline & Gamma dpa/s & $9.97 \mathrm{E}-13$ & 49 & $1.05 \mathrm{E}-12$ & 16 & 1.05 & & 1.00 \\
\hline & Gamma flux & $3.17 \mathrm{E}+12$ & 52 & $3.33 \mathrm{E}+12$ & 30 & 1.05 & & 1.00 \\
\hline & Neutron + gamma dpa/s & $4.98 \mathrm{E}-12$ & & $5.53 \mathrm{E}-12$ & 11 & 1.11 & & 0.98 \\
\hline \multirow{6}{*}{ Position 3} & Neutron flux $(\mathrm{E}>1 \mathrm{MeV})$ & $2.32 \mathrm{E}+09$ & 53 & $3.95 \mathrm{E}+09$ & 16 & 1.71 & \multirow[t]{6}{*}{1.70} & 1.01 \\
\hline & Neutron flux $(\mathrm{E}>0.5 \mathrm{MeV})$ & $3.91 \mathrm{E}+09$ & 53 & $6.70 \mathrm{E}+09$ & 18 & 1.71 & & 1.00 \\
\hline & Neutron flux $(\mathrm{E}>0.1 \mathrm{MeV})$ & $6.39 \mathrm{E}+09$ & 52 & $1.10 \mathrm{E}+10$ & 21 & 1.73 & & 0.99 \\
\hline & Neutron flux $(\mathrm{E}<0.414 \mathrm{eV})$ & $4.27 \mathrm{E}+10$ & 55 & $1.13 \mathrm{E}+11$ & 7 & 2.63 & & 1.00 \\
\hline & $\mathrm{dpa} / \mathrm{s}(\mathrm{Fe}, \mathrm{ASTM})$ & $3.99 \mathrm{E}-12$ & 47 & $7.18 \mathrm{E}-12$ & 13 & 1.80 & & 0.99 \\
\hline & $\mathrm{dpa} / \mathrm{s}(\mathrm{Fe}, \mathrm{ENDF}-\mathrm{VI})$ & $3.96 \mathrm{E}-12$ & 47 & $7.10 \mathrm{E}-12$ & 14 & 1.80 & & 0.99 \\
\hline
\end{tabular}

${ }^{a}$ Units are $\mathrm{cm}^{-2} \mathrm{~s}^{-1}$ for neutron and gamma-ray fluxes and $\mathrm{s}^{-1}$ for $\mathrm{dpa} / \mathrm{s}$.

${ }^{b}$ Percent standard deviation. The $\chi 2$ per degree of freedom for the adjustment run was 9.584E-01.

"New" adjustment was done with calculated fluxes for each of the three positions; "old" adjustment was done with calculated fluxes for Position 2 only. 
Table D.6. Calculated (for Position 2 only) and adjusted irradiation parameters for capsule DOS-5, at K3, P10 from the preliminary analysis and comparison with the final analysis

\begin{tabular}{|c|c|c|c|c|c|c|c|c|}
\hline & Irradiation parameter & $\begin{array}{c}\text { Calculated } \\
\text { value } \\
()^{a}\end{array}$ & $\begin{array}{l}\text { Std. }^{b} \\
(\%)\end{array}$ & $\begin{array}{c}\text { Adjusted } \\
\text { value } \\
()^{a}\end{array}$ & $\begin{array}{l}\text { Std. }^{b} \\
(\%)\end{array}$ & $\begin{array}{l}\text { Adj./ } \\
\text { calc. }\end{array}$ & $\begin{array}{c}\text { Ni58(n,p) } \\
\text { M/C }\end{array}$ & $\begin{array}{l}\text { Adjusted } \\
\text { new/old }^{c}\end{array}$ \\
\hline \multirow{6}{*}{ Position 1} & Neutron flux $(\mathrm{E}>1 \mathrm{MeV})$ & $1.72 \mathrm{E}+09$ & 34 & $1.22 \mathrm{E}+09$ & 10 & 0.71 & \multirow[t]{6}{*}{0.76} & 0.92 \\
\hline & Neutron flux $(\mathrm{E}>0.5 \mathrm{MeV})$ & $2.98 \mathrm{E}+09$ & 34 & $2.11 \mathrm{E}+09$ & 11 & 0.71 & & 0.91 \\
\hline & Neutron flux $(\mathrm{E}>0.1 \mathrm{MeV})$ & $4.95 \mathrm{E}+09$ & 33 & $3.47 \mathrm{E}+09$ & 13 & 0.70 & & 0.91 \\
\hline & Neutron flux $(\mathrm{E}<0.414 \mathrm{eV})$ & $3.23 \mathrm{E}+10$ & 40 & $2.11 \mathrm{E}+10$ & 4 & 0.65 & & 0.99 \\
\hline & $\mathrm{dpa} / \mathrm{s}(\mathrm{Fe}, \mathrm{ASTM})$ & $3.00 \mathrm{E}-12$ & 30 & $2.11 \mathrm{E}-12$ & 9 & 0.70 & & 0.94 \\
\hline & $\mathrm{dpa} / \mathrm{s} \quad(\mathrm{Fe}, \mathrm{ENDF}-\mathrm{VI})$ & $2.98 \mathrm{E}-12$ & 30 & $2.09 \mathrm{E}-12$ & 9 & 0.70 & & 0.94 \\
\hline \multirow{9}{*}{ Position 2} & Neutron flux $(\mathrm{E}>1 \mathrm{MeV})$ & $1.72 \mathrm{E}+09$ & 34 & $1.81 \mathrm{E}+09$ & 10 & 1.05 & \multirow[t]{9}{*}{1.14} & 1.00 \\
\hline & Neutron flux $(\mathrm{E}>0.5 \mathrm{MeV})$ & $2.98 \mathrm{E}+09$ & 34 & $3.12 \mathrm{E}+09$ & 11 & 1.05 & & 1.00 \\
\hline & Neutron flux $(\mathrm{E}>0.1 \mathrm{MeV})$ & $4.95 \mathrm{E}+09$ & 33 & $5.13 \mathrm{E}+09$ & 12 & 1.04 & & 1.00 \\
\hline & Neutron flux $(\mathrm{E}<0.414 \mathrm{eV})$ & $3.23 \mathrm{E}+10$ & 40 & $3.89 \mathrm{E}+10$ & 25 & 1.20 & & 0.96 \\
\hline & $\mathrm{dpa} / \mathrm{s}(\mathrm{Fe}, \mathrm{ASTM})$ & $3.00 \mathrm{E}-12$ & 30 & $3.18 \mathrm{E}-12$ & 9 & 1.06 & & 0.99 \\
\hline & $\mathrm{dpa} / \mathrm{s}(\mathrm{Fe}, \mathrm{ENDF}-\mathrm{VI})$ & $2.98 \mathrm{E}-12$ & 30 & $3.16 \mathrm{E}-12$ & 9 & 1.06 & & 0.99 \\
\hline & Gamma dpa/s & $9.88 \mathrm{E}-13$ & 31 & $9.40 \mathrm{E}-13$ & 9 & 0.95 & & 1.00 \\
\hline & Gamma flux & $3.47 \mathrm{E}+12$ & 33 & $3.56 \mathrm{E}+12$ & 19 & 1.03 & & 1.00 \\
\hline & Neutron + gamma dpa/s & $3.99 \mathrm{E}-12$ & & $4.12 \mathrm{E}-12$ & 7 & 1.03 & & 0.99 \\
\hline \multirow{6}{*}{ Position 3} & Neutron flux (E > 1MeV) & $1.72 \mathrm{E}+09$ & 34 & $2.85 \mathrm{E}+09$ & 10 & 1.65 & \multirow[t]{6}{*}{1.74} & 1.04 \\
\hline & Neutron flux $(\mathrm{E}>0.5 \mathrm{MeV})$ & $2.98 \mathrm{E}+09$ & 34 & $4.94 \mathrm{E}+09$ & 11 & 1.66 & & 1.04 \\
\hline & Neutron flux $(\mathrm{E}>0.1 \mathrm{MeV})$ & $4.95 \mathrm{E}+09$ & 33 & $8.27 \mathrm{E}+09$ & 13 & 1.67 & & 1.03 \\
\hline & Neutron flux $(\mathrm{E}<0.414 \mathrm{eV})$ & $3.23 \mathrm{E}+10$ & 40 & $1.06 \mathrm{E}+11$ & 4 & 3.28 & & 1.01 \\
\hline & $\mathrm{dpa} / \mathrm{s}(\mathrm{Fe}, \mathrm{ASTM})$ & $3.00 \mathrm{E}-12$ & 30 & $5.50 \mathrm{E}-12$ & 9 & 1.83 & & 1.02 \\
\hline & $\mathrm{dpa} / \mathrm{s}(\mathrm{Fe}, \mathrm{ENDF}-\mathrm{VI})$ & $2.98 \mathrm{E}-12$ & 30 & $5.43 \mathrm{E}-12$ & 9 & 1.82 & & 1.02 \\
\hline
\end{tabular}

${ }^{a}$ Units are $\mathrm{cm}^{-2} \mathrm{~s}^{-1}$ for neutron and gamma-ray fluxes and $\mathrm{s}^{-1}$ for $\mathrm{dpa} / \mathrm{s}$.

${ }^{b}$ Percent standard deviation. The $\chi 2$ per degree of freedom for the adjustment run was 9.315E-01.

"New" adjustment was done with calculated fluxes for each of the three positions; "old" adjustment was done with calculated fluxes for Position 2 only. 



\section{APPENDIX E}

EFFECT OF NEUTRON AND GAMMA FLUX CHANGES 

In the adjustment calculations at certain locations the calculated fluxes were the arithmetic averages of the beginning-of-the-fuel-cycle (BOC) and the end-of-the-fuel-cycle (EOC) fluxes, while at some locations the fluxes calculated for EOC were used.

To assess the impact of this approximation on the adjusted irradiation parameters, the adjustment was repeated for three selected locations of dosimeters, with BOC and EOC neutron and gamma fluxes and the results are compared with the adjustment in which the average fluxes were used.

Table E.1 gives the calculated neutron and gamma fluxes for the BOC and EOC, together with the average fluxes for the location 2D3W. Table E.2 gives the same information for the locations $2 \mathrm{D} 1 \mathrm{C}$ and 2D2C. These locations were selected because they are important for the pressure vessel surveillance. Tables E.3-E.5 compare the adjusted irradiation parameters from the three adjustment runs: one performed with the average neutron and gamma fluxes, one with the BOC fluxes, and one with EOC fluxes, for the three locations considered.

The differences between the adjusted irradiation parameters from the run with the average fluxes and the two runs with BOC and EOC fluxes typically do not exceed 1\%, except for the gamma induced dpa and gamma flux at the location $2 \mathrm{D} 2 \mathrm{C}$ where the differences reach about $7 \%$. In all cases the differences are much smaller than the standard deviations of the adjusted parameters. Therefore, the use of the average fluxes in the adjustment procedure is justified. 
Table E.1. Calculated neutron and gamma fluxes at the location 2D3W.

The fluxes are given at the beginning-of-the-fuel-cycle (BOC) and at the end-of-the-fuel-cycle (EOC).

Arithmetic averages of the BOC and EOC fluxes are given also. Units are $\left(\mathrm{cm}^{-2} \mathrm{~s}^{-1}\right)$.

\section{BOC neutron and gamma fluxes}

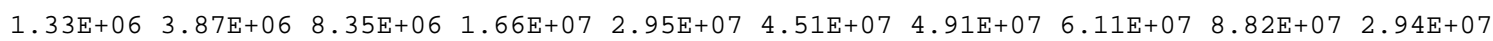

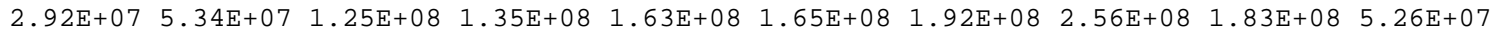

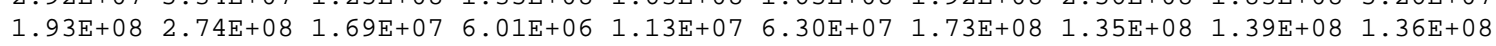

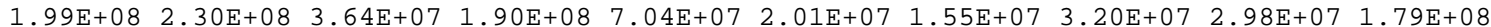

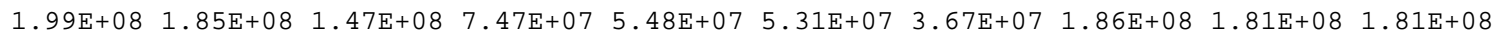

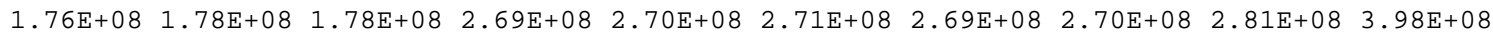
$1.05 \mathrm{E}+10$

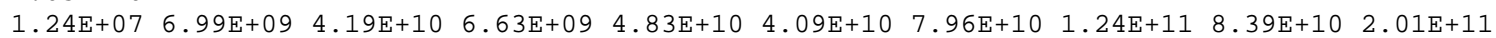
$\begin{array}{lllllllllll}1.75 \mathrm{E}+11 & 2.42 \mathrm{E}+11 & 2.25 \mathrm{E}+11 & 1.02 \mathrm{E}+11 & 1.75 \mathrm{E}+11 & 2.12 \mathrm{E}+11 & 2.82 \mathrm{E}+11 & 9.85 \mathrm{E}+11 & 5.67 \mathrm{E}+11 & 4.23 \mathrm{E}+11\end{array}$ 3. 35E+11 5.06E+10 1.86E+08

\section{EOC neutron and gamma fluxes}

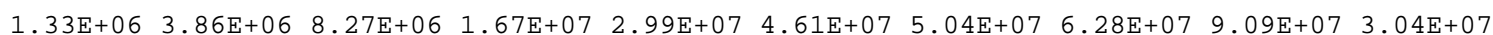

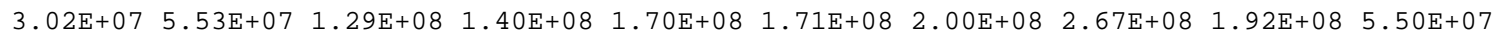

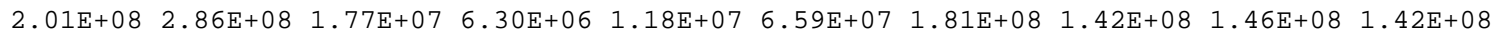
$\begin{array}{llllllllll}2.08 \mathrm{E}+08 & 2.41 \mathrm{E}+08 & 3.81 \mathrm{E}+07 & 2.00 \mathrm{E}+08 & 7.37 \mathrm{E}+07 & 2.10 \mathrm{E}+07 & 1.62 \mathrm{E}+07 & 3.36 \mathrm{E}+07 & 3.13 \mathrm{E}+07 & 1.88 \mathrm{E}+08\end{array}$

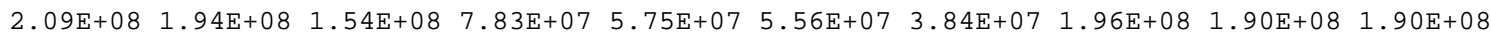

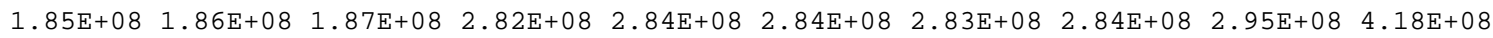
$1.13 \mathrm{E}+10$

1.44E+07 8.46E+09 5.88E+10 8.83E+09 $6.48 \mathrm{E}+10 \quad 5.01 \mathrm{E}+10 \quad 8.73 \mathrm{E}+10 \quad 1.30 \mathrm{E}+11 \quad 8.62 \mathrm{E}+10 \quad 2.23 \mathrm{E}+11$

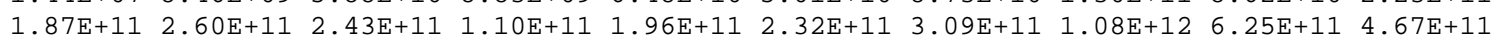

3. $70 \mathrm{E}+11 \quad 5.59 \mathrm{E}+10 \quad 2.05 \mathrm{E}+08$

\section{Average neutron and gamma fluxes}

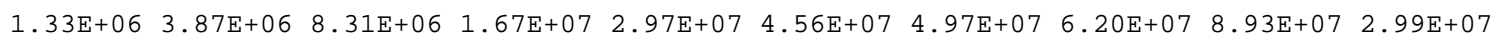

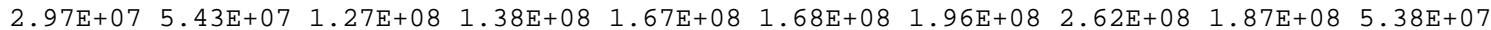

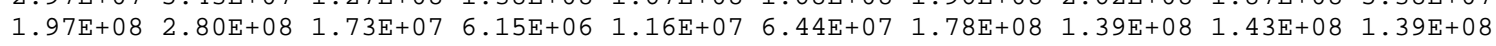
$\begin{array}{lllllllllll}2.04 \mathrm{E}+08 & 2.35 \mathrm{E}+08 & 3.72 \mathrm{E}+07 & 1.95 \mathrm{E}+08 & 7.20 \mathrm{E}+07 & 2.06 \mathrm{E}+07 & 1.59 \mathrm{E}+07 & 3.27 \mathrm{E}+07 & 3.05 \mathrm{E}+07 & 1.84 \mathrm{E}+08\end{array}$

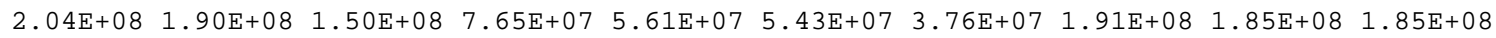

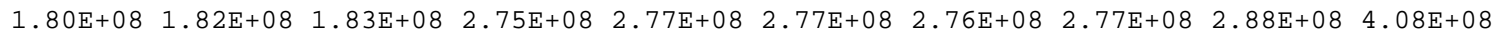
$1.09 \mathrm{E}+10$

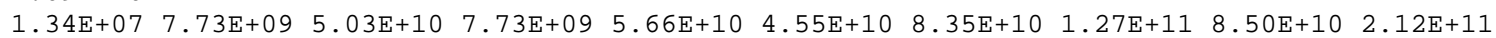
$\begin{array}{lllllllllll}1.81 \mathrm{E}+11 & 2.52 \mathrm{E}+11 & 2.35 \mathrm{E}+11 & 1.06 \mathrm{E}+11 & 1.85 \mathrm{E}+11 & 2.22 \mathrm{E}+11 & 2.96 \mathrm{E}+11 & 1.04 \mathrm{E}+12 & 5.96 \mathrm{E}+11 & 4.45 \mathrm{E}+11\end{array}$ $3.53 \mathrm{E}+11$ 5.33E+10 $1.96 \mathrm{E}+08$ 
Table E.2. Calculated neutron and gamma fluxes at the locations 2D1C and 2D2C.

The fluxes are given at the beginning-of-the-fuel-cycle (BOC) and at the end-of-the-fuel-cycle (EOC).

Arithmetic averages of the BOC and EOC fluxes are given also. Units are $\left(\mathrm{cm}^{-2} \mathrm{~s}^{-1}\right)$.

\section{BOC neutron and gamma fluxes}

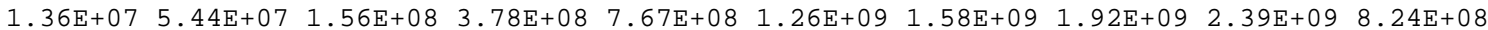

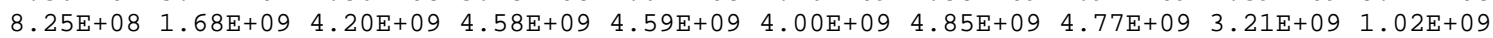

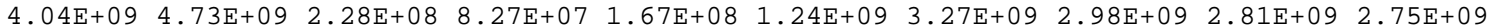

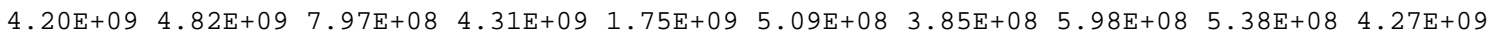

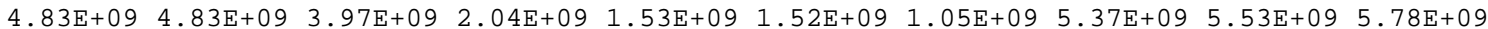

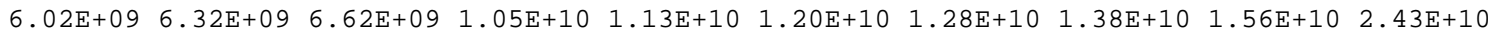
2. $04 \mathrm{E}+12$

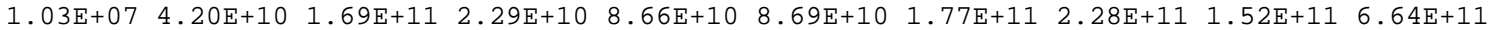

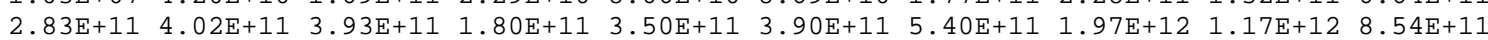

$5.06 \mathrm{E}+11 \quad 6.95 \mathrm{E}+10 \quad 2.53 \mathrm{E}+08$

\section{EOC neutron and gamma fluxes}

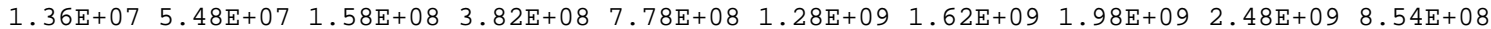
8.54E+08 1.74E+09 4.39E+09 4.80E+09 4.82E+09 4.20E+09 $5.11 \mathrm{E}+09 \quad 5.03 \mathrm{E}+09 \quad 3.39 \mathrm{E}+09 \quad 1.08 \mathrm{E}+09$

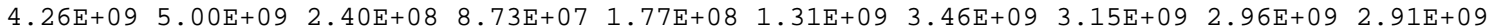

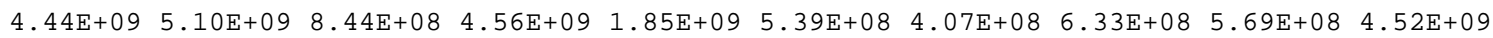

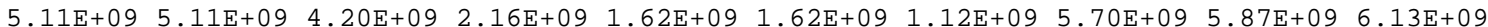

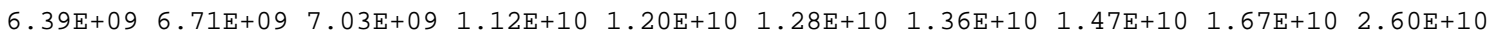
2. $46 \mathrm{E}+12$

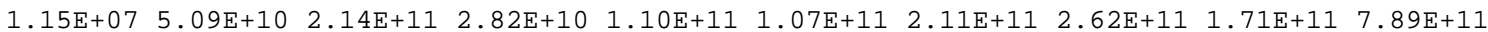

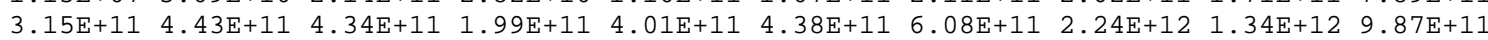
$5.87 \mathrm{E}+11 \quad 8.08 \mathrm{E}+10 \quad 2.94 \mathrm{E}+08$

\section{Average neutron and gamma fluxes}

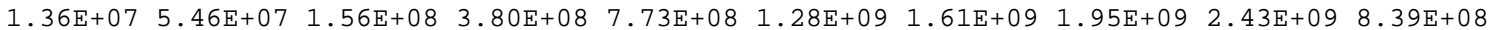

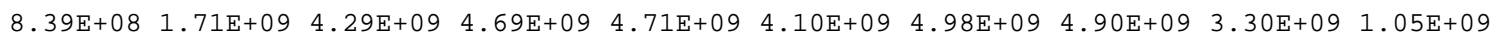

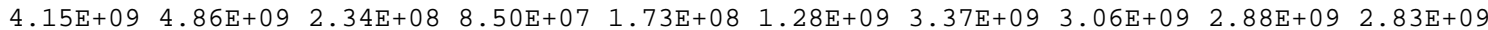

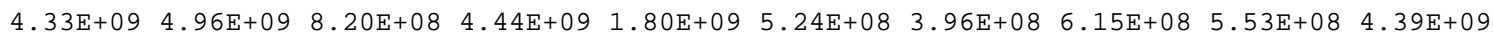

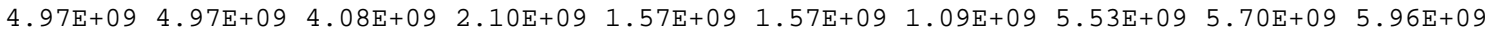

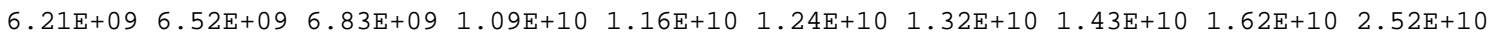
$2.25 \mathrm{E}+12$

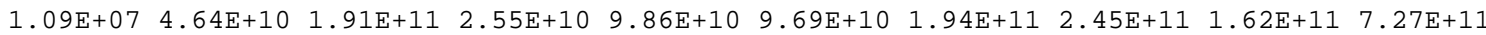

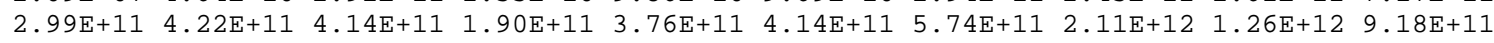
$5.47 \mathrm{E}+11 \quad 7.51 \mathrm{E}+10 \quad 2.74 \mathrm{E}+08$ 
Table E.3. Calculated and adjusted irradiation parameters at 2D3W (capsules HRB2-3G and HRB2-3A, position 2; dosimeter sets GD-18 and AL-31; HRB2-3W). Adjustment is performed with the average, BOC, and EOC neutron and gamma fluxes.

\begin{tabular}{|c|c|c|c|c|c|c|c|c|}
\hline \multirow[b]{2}{*}{ Irradiation parameter $^{a}$} & \multicolumn{2}{|c|}{ Unadjusted value } & \multicolumn{2}{|c|}{ Average fluxes } & \multicolumn{2}{|c|}{ BOC fluxes } & \multicolumn{2}{|c|}{ EOC fluxes } \\
\hline & $\begin{array}{c}\text { Calculated } \\
\text { value } \\
()^{b}\end{array}$ & $\begin{array}{l}\text { Std. }{ }^{c} \\
(\%)\end{array}$ & $\begin{array}{c}\text { Adjusted } \\
\text { value } \\
()^{b}\end{array}$ & $\begin{array}{l}\text { Std. }^{c} \\
(\%)\end{array}$ & $\begin{array}{c}\text { Adjusted } \\
\text { value } \\
()^{b}\end{array}$ & $\begin{array}{l}\text { Std. }{ }^{c} \\
(\%)\end{array}$ & $\begin{array}{c}\text { Adjusted } \\
\text { value } \\
()^{b}\end{array}$ & $\begin{array}{l}\text { Std. }^{c} \\
\text { (\%) }\end{array}$ \\
\hline Neutron flux $(\mathrm{E}>1 \mathrm{MeV})$ & $9.42 \mathrm{E}+08$ & 19 & $1.10 \mathrm{E}+09$ & 6 & $1.10 \mathrm{E}+09$ & 6 & $1.10 \mathrm{E}+10$ & 6 \\
\hline Neutron flux $(E>0.5 \mathrm{MeV})$ & $1.72 \mathrm{E}+09$ & 19 & $1.99 \mathrm{E}+09$ & 7 & $1.99 \mathrm{E}+09$ & 7 & $2.00 \mathrm{E}+09$ & 7 \\
\hline Neutron flux $(\mathrm{E}>0.1 \mathrm{MeV})$ & $3.01 \mathrm{E}+09$ & 19 & $3.47 \mathrm{E}+09$ & 8 & $3.46 \mathrm{E}+09$ & 8 & $3.49 \mathrm{E}+09$ & 8 \\
\hline Neutron flux $(\mathrm{E}<0.414 \mathrm{eV})$ & $1.09 \mathrm{E}+10$ & 40 & $1.39 \mathrm{E}+10$ & 6 & $1.39 \mathrm{E}+10$ & 6 & $1.39 \mathrm{E}+10$ & 6 \\
\hline Neutron dpa/s (Fe, ASTM) & $1.71 \mathrm{E}-12$ & 18 & $1.99 \mathrm{E}-12$ & 6 & $1.98 \mathrm{E}-12$ & 6 & $1.99 \mathrm{E}-12$ & 6 \\
\hline Neutron dpa/s (Fe, ENDF-VI) & $1.71 \mathrm{E}-12$ & 18 & $1.99 \mathrm{E}-12$ & 6 & $1.98 \mathrm{E}-12$ & 6 & $1.99 \mathrm{E}-12$ & 6 \\
\hline Gamma dpa/s & $1.01 \mathrm{E}-12$ & 27 & $1.18 \mathrm{E}-12$ & 7 & $1.18 \mathrm{E}-12$ & 7 & $1.18 \mathrm{E}-12$ & 7 \\
\hline Gamma flux & $4.64 \mathrm{E}+12$ & 28 & $5.33 \mathrm{E}+12$ & 16 & $5.34 \mathrm{E}+12$ & 16 & $5.32 \mathrm{E}+12$ & 17 \\
\hline Neutron + gamma dpa/s & $2.72 \mathrm{E}-12$ & & $3.17 \mathrm{E}-12$ & 4 & $3.16 \mathrm{E}-12$ & 4 & $3.18 \mathrm{E}-12$ & 4 \\
\hline
\end{tabular}

The measurements from cycles 400 and 401 were treated together in the adjustment.

${ }^{b}$ Units are $\mathrm{cm}^{-2} \mathrm{~s}^{-1}$ for neutron and gamma-ray fluxes and $\mathrm{s}^{-1}$ for $\mathrm{dpa} / \mathrm{s}$.

${ }^{c}$ Percent standard deviation. 
Table E.4. Calculated and adjusted irradiation parameters at 2D1C (capsules HRB2-1G and HRB2-1A, position 2; dosimeter sets GD-14 and AL-27; HRB2-1C). Adjustment is performed with the average, BOC, and EOC neutron and gamma fluxes.

\begin{tabular}{|c|c|c|c|c|c|c|c|c|}
\hline \multirow[b]{2}{*}{ Irradiation parameter $^{a}$} & \multicolumn{2}{|c|}{ Unadjusted value } & \multicolumn{2}{|c|}{ Average fluxes } & \multicolumn{2}{|c|}{ BOC fluxes } & \multicolumn{2}{|c|}{ EOC fluxes } \\
\hline & $\begin{array}{l}\text { Calculated } \\
\text { value } \\
()^{b}\end{array}$ & $\begin{array}{l}\text { Std. }^{c} \\
(\%)\end{array}$ & $\begin{array}{c}\text { Adjusted } \\
\text { value } \\
()^{b}\end{array}$ & $\begin{array}{l}\text { Std. }^{c} \\
(\%)\end{array}$ & $\begin{array}{c}\text { Adjusted } \\
\text { value } \\
()^{b}\end{array}$ & $\begin{array}{l}\text { Std. }{ }^{c} \\
(\%)\end{array}$ & $\begin{array}{c}\text { Adjusted } \\
\text { value } \\
()^{b}\end{array}$ & $\begin{array}{l}\text { Std. }{ }^{c} \\
(\%)\end{array}$ \\
\hline Neutron flux $(\mathrm{E}>1 \mathrm{MeV})$ & $2.79 \mathrm{E}+10$ & 63 & $2.23 \mathrm{E}+10$ & 12 & $2.25 \mathrm{E}+10$ & 11 & $2.22 \mathrm{E}+10$ & 12 \\
\hline Neutron flux $(E>0.5 \mathrm{MeV})$ & $4.40 \mathrm{E}+10$ & 63 & $3.41 \mathrm{E}+10$ & 13 & $3.44 \mathrm{E}+10$ & 13 & $3.39 \mathrm{E}+10$ & 13 \\
\hline Neutron flux $(E>0.1 \mathrm{MeV})$ & $6.95 \mathrm{E}+10$ & 62 & $5.18 \mathrm{E}+10$ & 15 & $5.24 \mathrm{E}+10$ & 15 & $5.15 \mathrm{E}+10$ & 16 \\
\hline Neutron flux $(\mathrm{E}<0.414 \mathrm{eV})$ & $2.25 \mathrm{E}+12$ & 65 & $1.43 \mathrm{E}+12$ & 6 & $1.43 \mathrm{E}+12$ & 6 & $1.43 \mathrm{E}+12$ & 6 \\
\hline Neutron dpa/s (Fe, ASTM) & $6.10 \mathrm{E}-11$ & 48 & $4.48 \mathrm{E}-11$ & 7 & $4.50 \mathrm{E}-11$ & 7 & $4.46 \mathrm{E}-11$ & 7 \\
\hline Neutron dpa/s (Fe, ENDF-VI) & $5.92 \mathrm{E}-11$ & 49 & $4.35 \mathrm{E}-11$ & 7 & $4.38 \mathrm{E}-11$ & 7 & $4.34 \mathrm{E}-11$ & 7 \\
\hline Gamma dpa/s & $2.76 \mathrm{E}-12$ & 58 & $6.13 \mathrm{E}-12$ & 31 & $6.11 \mathrm{E}-12$ & 32 & $6.11 \mathrm{E}-12$ & 29 \\
\hline Gamma flux & $9.39 \mathrm{E}+12$ & 61 & $2.10 \mathrm{E}+13$ & 44 & $2.11 \mathrm{E}+13$ & 44 & $2.08 \mathrm{E}+13$ & 43 \\
\hline Neutron + gamma dpa/s & $6.38 \mathrm{E}-11$ & & $5.09 \mathrm{E}-11$ & 7 & $5.11 \mathrm{E}-11$ & 7 & $5.07 \mathrm{E}-11$ & 7 \\
\hline
\end{tabular}

The measurements from cycles 400 and 401 were treated together in the adjustment.

${ }^{b}$ Units are $\mathrm{cm}^{-2} \mathrm{~s}^{-1}$ for neutron and gamma-ray fluxes and $\mathrm{s}^{-1}$ for $\mathrm{dpa} / \mathrm{s}$.

${ }^{c}$ Percent standard deviation. 
Table E.5. Calculated and adjusted irradiation parameters at 2D2C (capsules HRB2-2G and HRB2-2A, position 2; dosimeter sets GD-16 and AL-29; HRB2-2C). Adjustment is performed with the average, BOC, and EOC neutron and gamma fluxes.

\begin{tabular}{|c|c|c|c|c|c|c|c|c|}
\hline \multirow[b]{2}{*}{ Irradiation parameter $^{a}$} & \multicolumn{2}{|c|}{ Unadjusted value } & \multicolumn{2}{|c|}{ Average fluxes } & \multicolumn{2}{|c|}{ BOC fluxes } & \multicolumn{2}{|c|}{ EOC fluxes } \\
\hline & $\begin{array}{c}\text { Calculated } \\
\text { value } \\
()^{b}\end{array}$ & $\begin{array}{l}\text { Std. }^{c} \\
(\%)\end{array}$ & $\begin{array}{c}\text { Adjusted } \\
\text { value } \\
()^{b}\end{array}$ & $\begin{array}{l}\text { Std. }^{c} \\
(\%)\end{array}$ & $\begin{array}{c}\text { Adjusted } \\
\text { value } \\
()^{b}\end{array}$ & $\begin{array}{l}\text { Std. }{ }^{c} \\
(\%)\end{array}$ & $\begin{array}{c}\text { Adjusted } \\
\text { value } \\
()^{b}\end{array}$ & $\begin{array}{l}\text { Std. }^{c} \\
\text { (\%) }\end{array}$ \\
\hline Neutron flux $(\mathrm{E}>1 \mathrm{MeV})$ & $2.79 \mathrm{E}+10$ & 19 & $2.99 \mathrm{E}+10$ & 6 & $2.99 \mathrm{E}+10$ & 6 & $3.00 \mathrm{E}+10$ & 6 \\
\hline Neutron flux $(E>0.5 \mathrm{MeV})$ & $4.40 \mathrm{E}+10$ & 19 & $4.70 \mathrm{E}+10$ & 6 & $4.70 \mathrm{E}+10$ & 6 & $4.73 \mathrm{E}+10$ & 6 \\
\hline Neutron flux $(\mathrm{E}>0.1 \mathrm{MeV})$ & $6.95 \mathrm{E}+10$ & 19 & $7.40 \mathrm{E}+10$ & 7 & $7.39 \mathrm{E}+10$ & 7 & $7.44 \mathrm{E}+10$ & 7 \\
\hline Neutron flux $(\mathrm{E}<0.414 \mathrm{eV})$ & $2.25 \mathrm{E}+12$ & 20 & $2.08 \mathrm{E}+12$ & 6 & $2.06 \mathrm{E}+12$ & 6 & $2.09 \mathrm{E}+12$ & 6 \\
\hline Neutron dpa/s (Fe, ASTM) & $6.10 \mathrm{E}-11$ & 15 & $6.24 \mathrm{E}-11$ & 4 & $6.22 \mathrm{E}-11$ & 4 & $6.26 \mathrm{E}-11$ & 4 \\
\hline Neutron dpa/s (Fe, ENDF-VI) & $5.92 \mathrm{E}-11$ & 15 & $6.07 \mathrm{E}-11$ & 4 & $6.05 \mathrm{E}-11$ & 4 & $6.09 \mathrm{E}-11$ & 4 \\
\hline Gamma dpa/s & $2.76 \mathrm{E}-12$ & 27 & $3.23 \mathrm{E}-12$ & 21 & $3.02 \mathrm{E}-12$ & 21 & $3.42 \mathrm{E}-12$ & 20 \\
\hline Gamma flux & $9.39 \mathrm{E}+12$ & 28 & $1.09 \mathrm{E}+13$ & 24 & $1.04 \mathrm{E}+13$ & 25 & $1.13 \mathrm{E}+13$ & 24 \\
\hline Neutron + gamma dpa/s & $6.38 \mathrm{E}-11$ & & $6.56 \mathrm{E}-11$ & 4 & $6.52 \mathrm{E}-11$ & 4 & $6.61 \mathrm{E}-11$ & 4 \\
\hline
\end{tabular}

The measurements from cycles 400 and 401 were treated together in the adjustment.

${ }^{b}$ Units are $\mathrm{cm}^{-2} \mathrm{~s}^{-1}$ for neutron and gamma-ray fluxes and $\mathrm{s}^{-1}$ for $\mathrm{dpa} / \mathrm{s}$.

${ }^{c}$ Percent standard deviation. 


\section{APPENDIX F}

COMPUTER CODES AND DATA ARCHIVE 

This appendix summarizes information about the computer codes used for the analysis of the dosimeters and the information on the computer runs performed.

The information is archived on DVD (labeled Dosimetry and Charpy Analysis CY400-401) and in the HFIR directory on CPILE, in the directory CY400-401-Dosimetry.

The archive consists of three main directories: Codes, which contains the information on the codes used; Dosimetry, which contains all the data used to analyze the dosimetry experiments; and Charpy, which contains information on the dosimetry analysis from the Charpy capsules.

The "Codes" directory contains the source codes in the subdirectory, "src," executables in the subdirectory, "bin," a few auxiliary scripts used for file manipulation in "scripts," and a subdirectory "verification," which contains an example of the adjustment run, used to demonstrate that the code LSLM2 installed on CPILE under the Linux operating system produces the same results as the original LSLM2 code, which was designed for use with a DOS operating system. The details about the computer system, operating system, and compiler are listed in Table F.1.

Table F.1. Computer and code information

\begin{tabular}{ll}
\hline Computer system name & CPILE \\
Computer type/model & KC-Computer Co., "IBM" compatible \\
Operating system/version & Red Hat Linux 7.3 \\
Computer code/version & LSLM2 \\
Type of compiler & LINUX Portland Group FORTRAN 4.0.2 \\
Owner of code & ORNL \\
RSICC ${ }^{a}$ distribution number & PSR-233 MICRO \\
\hline${ }^{a}$ Radiation Safety Information Computational Center, located and operated \\
within the Nuclear Science and Technology Division at Oak Ridge National \\
Laboratory.
\end{tabular}

The LSLM2 computer code package consists of four codes. The main code is the "LSLM2," which performs a least-squares logarithmic adjustment of neutron and gamma spectra based on the measured reaction rates. The program, "ACT," performs conversion from dosimeter activities to reactions rates. Program "CALACT" calculates reaction rates from the reaction rate cross sections and neutron and gamma fluxes. The last program, "FLXPRO," performs conversion of group fluxes, cross sections, and covariances from one energy group structure to another. The codes are written in FORTRAN-77.

The archive directory, "Dosimetry," contains all the data and computer runs performed to analyze the dosimetry experiments performed in HFIR fuel cycles 400 and 401 . The list of the subdirectories and their content is listed in Table F.2. 
Table F.2. Content of the archive directory, "Dosimetry"

\begin{tabular}{ll}
\hline \multicolumn{1}{c}{ Subdirectory } & \multicolumn{1}{c}{ Content } \\
\hline Cross-sections & Reaction cross sections and manipulation runs \\
Fluxes & Neutron and gamma fluxes obtained from transport calculations \\
Calculated-rr & $\begin{array}{l}\text { Reaction rate calculated from neutron and gamma fluxes } \\
\text { and reaction cross sections }\end{array}$ \\
Calculated-rr-gamma & Calculated gamma-ray induced reaction rates \\
Measured-rr & Measured specific activities and 'measured" reaction rates \\
Spv & Preparation of the covariance matrix for neutron and gamma fluxes \\
Power-history & Power history of HFIR operation \\
Adjustment & All adjustment runs with the LSLM2 computer code \\
\hline
\end{tabular}

The directory, "Adjustment," contains one subdirectory for every location where the adjusted irradiation parameters are provided in Table 4 of this report. Each subdirectory contains another subdirectory, named, for example, "Report-Table-5," indicating that this subdirectory contains the adjustment run, which provided results for the Table 5 of the report. Additional subdirectories are given if more adjustment runs were discussed in the report, and the subdirectory, "Archive," contains all other adjustment runs performed to evaluate the impact of different parameters on the adjusted irradiation parameters, but not specifically listed in the report.

Throughout the archive there are "README" files, which provide additional explanation of the archived data. The archived data and codes provide complete information necessary to reproduce the analysis. 


\section{INTERNAL DISTRIBUTION}

1. C. A. Baldwin

2. E. D. Blakeman

3-4. R. D. Cheverton

5. T. L. Dickson

6. P. D. Ferguson

7. D. C. Glasgow

8-9. S. K. Iskander

10-14. H. A. Kmieciak, Jr.

15. R. K. Nanstad

16. C. V. Parks
17. I. Remec

18. J. E. Rushton

19. C. O. Slater

20. K. A. Smith

21. M. A. Sokolov

22. J. C. Wagner

23. Central Research Library

24. ORNL Laboratory Records

25-26. ORNL Laboratory Records-OSTI

27-28. RRD-DCC-RC 